\title{
Bone Research Society Abstracts
}

$T_{\text {o }}^{\text {he }}$ he Bone Research Society (BRS; www.boneresearchsociety. org), formerly the Bone and Tooth Society, was founded in 1950. The BRS is one of the largest national scientific societies in Europe dedicated to clinical and basic research into mineralized tissues and is the oldest such society in the world. Meetings are held annually, attracting a wide audience from throughout the UK and beyond. The presentations are traditionally balanced between clinical and laboratory studies. The participation of young scientists and clinicians is actively encouraged.

The Annual Meeting this year was organized by a team from The University of Southampton, co-chaired by $\mathrm{Dr}$ Kate Ward and Professor Nicholas Harvey, and also including Dr Claire Clarkin, Professor Richard Oreffo, and Professor Cyrus Cooper. The program brought together world leaders in musculoskeletal clinical and basic science. A multidisciplinary faculty provided a breadth of education and research from the Developmental Origins of disease through tissue regeneration and biomechanical engineering.

The sessions this year were:

Rare bone diseases

Muscle bone interactions

Tissue engineering and regenerative medicine

Cellular senescence

Imaging: from mice to men

Osteocytes

Prevention of osteoporotic fracture through the lifecourse (Dent Award-Cyrus Cooper)

Early-life origins of disease

Ageing

Osteo-angiogenic coupling

More than 120 abstracts were submitted, and those that were accepted and presented at the meeting are listed here.

\section{Invited Speaker Abstracts}

\section{IS1}

\section{Polymers for controlling and manipulating cells}

\section{Mark Bradley and the ERC ADREEM Team}

\section{School of Chemistry, University of Edinburgh, Edinburgh, UK}

I will describe the suite of tools and technologies developed within the Bradley group (typically with collaborators) that are focused on controlling or modulating cellular behavior-with a key vision being that the material itself should provide the cues and stimuli that drive cell fate and behavior. In my talk, I will describe a variety of high-throughput approaches developed in the group, from the use of combinatorial delivery strategies to microarray platforms that allow a deep analysis of how substrate morphology and structure drive cellular behavior.

\section{IS2 \\ Re-engineering developmental processes for tissue regeneration}

\section{Ivan Martin \\ Department of Biomedicine, University Hospital Basel, Basel, Switzerland}

Biological processes leading to tissue formation during embryonic development are characterized by a large stability and reproducibility of events, typically referred to as "robustness." Would regenerative medicine approaches be more repeatable and effective if they targeted the recapitulation of molecular pathways typical of tissue development? Within the exemplifying context of cartilage and bone repair, this lecture will introduce and discuss the challenges and opportunities of regenerative concepts based on mimicking developmental processes. Rather than engineering a tissue, the strategy would target the use of cells (eg, mesenchymal stromal cells) to engineer temporally staged processes, recapitulating events of development (eg, endochondral ossification for bone or joint cavitation for articular cartilage). The product would be a construct containing the necessary and sufficient cues to autonomously remodel into the target repair tissue upon grafting.

In this perspective, however, cells in adults may strongly differ from multipotent embryonic cells, and typically reside in an environment, which is tightly regulated by post-natal mechanical conditioning or immune/inflammatory processes. Thus, shouldn't tissue regeneration strategies be inspired by development but adapted to be effective in a context, which is different from the embryo? This would require the redesign of the developmental machinery for regenerative purposes by establishing artificial events or conditions. Will the resulting approach of "developmental re-engineering" offer a chance for enhanced regeneration to those tissues with limited capacity to recover from injuries or within pathological settings, reducing the potential for endogenous repair?

\section{IS3}

Mesenchymal stromal cell lineages in the joint in health, injury, and arthritis

\section{Anke Roelofs}

Institute of Medical Sciences, University of Aberdeen, Aberdeen, UK 
In recent years, skeletal stem and progenitor cell populations have been identified in bone marrow based on markers such as Nestin, Leptin receptor (LepR), and Gremlin1. Genetic lineage tracing models in mice have provided important insights in their roles in bone homeostasis, fracture repair, and hematopoiesis. More recently, the stem and progenitor cells that are resident in the synovial joint are beginning to be defined and their functions elucidated. Lineage tracing of progenitor cells in cartilage has provided important new insights into the process of articular cartilage formation and maintenance. Work in our lab has focused on characterizing stem and progenitor cells in the synovial membrane. During development, synovial joints form from a stripe of tissue in the limb bud that is characterized by expression of growth/differentiation factor 5 (Gdf5). Tracing of Gdf5-expressing cells showed that Gdf5-lineage cells persist in adult knee synovium up to at least 1 year of age. They express a range of mesenchymal stromal cell markers, such as Pdgfr $\alpha$ and Sca1, but show little overlap with cells expressing the skeletal stem/progenitor markers Nestin, LepR, and Gremlin1, suggesting they are a self-contained lineage within the skeletal system. FACS-sorted and culture-expanded Gdf5-lineage cells are highly chondrogenic but poorly osteogenic in vitro, and they promote cartilage repair upon orthotopic transplantation into cartilage defects of mice. Endogenously, Gdf5-lineage cells proliferate in synovium in response to traumatic cartilage injury, leading to synovial lining hyperplasia, and underpin spontaneous repair of cartilage defects. Both processes are dependent on the activity of the transcriptional co-factor Yes-associate protein (Yap) in these cells. In pathology, using the destabilization of the medial meniscus model of osteoarthritis, Gdf5-lineage cells were found to contribute to chondro-osteophyte formation and subchondral bone remodeling. In conclusion, recent findings are starting to unravel distinct mesenchymal stromal cell subsets in adult joint tissues, identifying the key players in joint pathophysiology and promising therapeutic targets for cartilage repair and treatment of osteoarthritis.

\section{IS4}

Targeting cellular senescence: a new therapeutic approach for age-related osteoporosis

\section{Sundeep Khosla}

Endocrinology and Kogod Center on Ageing, Mayo Clinic, Rochester, MN, USA

With the aging of the population and projected increase in osteoporotic fractures, coupled with the declining use of osteoporosis medications, there is a compelling need for new approaches to treat osteoporosis. Given that age-related osteoporosis generally coexists with multiple other comorbidities (eg, atherosclerosis, diabetes, frailty), all sharing aging itself as the leading risk factor, there is growing interest in the "Geroscience Hypothesis," which posits that manipulation of fundamental aging mechanisms will delay the appearance or severity of multiple chronic diseases because these diseases share the same underlying risk factor-age. In this context, one fundamental aging mechanism that has received considerable attention recently as contributing to multiple age-related morbidities is cellular senescence. There is now convincing evidence that senescent cells accumulate with age and drive age-related tissue dysfunction. Consistent with this, senescent cells have been shown to increase with aging in the bone microenvironment in mice and in humans. These cells produce a pro-inflammatory secretome that leads to increased bone resorption and decreased bone formation, and approaches that either eliminate senescent cells or impair the production of their pro-inflammatory secretome have been shown to prevent age-related bone loss in mice. Moreover, targeting senescent cells leads to a reduction in bone resorption and either a maintenance (trabecular bone) or increase (cortical bone) in bone formation, thus making this approach fundamentally different from conventional anti-resorptive therapy, which leads to a reduction in bone resorption and a coupled decrease in bone formation. Thus, targeting cellular senescence represents a novel therapeutic strategy to prevent not only bone loss but also potentially multiple age-related diseases simultaneously.

\section{IS5}

Label-free techniques and multimodal imaging in skeletal repair and regeneration

\author{
Sumeet Mahajan ${ }^{1,2}$ \\ ${ }^{1}$ Department of Chemistry, University of Southampton, \\ Southampton, UK \\ ${ }^{2}$ Institute for Life Sciences, University of Southampton, \\ Southampton, UK
}

In this talk, I would describe our work using label-free, noninvasive, and non-destructive techniques of Raman spectroscopy and multimodal non-linear microscopies such as coherent anti-Stokes Raman scattering (CARS) and second harmonic generation (SHG) in the area of skeletal repair and regeneration for quantitative understanding and objective assessment of cell behavior and tissue development. Using Raman spectroscopy, we have studied osteogenesis in primary bone cells from mice and humans. We characterized signatures of early osteoblast behavior by quantifying changes in DNA, phosphate species, and collagen matrix during different stages of osteogenic commitment. ${ }^{(1)}$ It was found the Raman spectroscopy could detect changes in phosphates earlier than the alkaline phosphatase assay as well as distinguish different species. We have further aimed to quantitatively understand and objectively assess cell behavior and tissue development for applications in skeletal repair and regeneration using multimodal techniques. We combine CARS, SHG, and two-photon excited autofluorescence (TPEF) on a single platform for simultaneous interrogation. These techniques image the chemical and structural composition allowing us to study the differentiation of skeletal stem cells (SSCs) and visualization of temporal changes accompanying adipogenesis ${ }^{(2)}$ and chondrogenesis ${ }^{(3)}$ in a completely label-free, non-invasive, and non-destructive way. We also verified both visually as well as through gene expression analysis that the SSC-differentiated live tissue constructs remain viable and are unaffected by CARS and SHG imaging Furthermore, elucidation of the architecture of the differentiated tissue is especially important for cartilage tissue engineering. The application of 3D in place of 2D imaging thus enabled us to obtain a comprehensive understanding of the collagen fiber network during the chondrogenic development of SSCs. Quantitation of the various molecular and structural readouts allowed us to develop assessment parameters to track the differentiation of SSCs and their in vitro tissue development. The 
non-invasive and non-destructive 3D imaging opens new avenues for real-time applications, while the label-free quantitation allows unprecedented insight and characterization of the development stages of skeletal "engineered-tissue" in the clinic for optimal use in therapy.

\section{References}

1. Smith SJ, Emery R, Pitsillides A, Clarkin CE, Mahajan S. Detection of early osteogenic commitment in primary cells using Raman spectroscopy. Analyst. 2017;142:1962-73.

2. Smus JP, Costa Moura C, McMorrow E, Tare RS, Oreffo ROC, Mahajan S. Tracking adipogenic differentiation of skeletal stem cells by label-free chemically selective imaging. Chem Sci. 2015;6:2282-6.

3. Moura CC, Lanham SA, Monfort T, Bourdakos KN, Tare RS, Oreffo ROC, Mahajan S. Quantitative temporal interrogation in $3 \mathrm{D}$ of bioengineered human cartilage using multimodal labelfree imaging [unpublished data].

\section{IS6}

Models for mechanobiology-image-based computational modelling approaches to understand the mechanical boundary conditions of musculoskeletal regeneration

\section{Markus O Heller}

\section{Department of Mechanical Engineering, University of Southampton, Southampton, UK}

It is well accepted that mechanics play an important role for the biological processes that occur during musculoskeletal regeneration and healing. The local mechanical environment is a result of a complex interaction between the soft and hard tissues at the organ, limb, and patient level of the musculoskeletal system as motion occurs. Identification of key parameters that influence and modify the internal loading conditions is therefore essential in order to better understand the mechanisms that govern and regulate musculoskeletal healing and regeneration. Computational models informed by imaging data provide an effective means for characterizing the mechanical environment. This talk will explore the fidelity of such computational methods in their application to human and animal models alike and discuss differences and similarities in their respective organ- and tissuelevel loading conditions. Providing a quantitative understanding of the mechanical boundary conditions across animals will provide the essential basis for unravelling the detailed mechanisms governing the biology of musculoskeletal regeneration.

\section{IS7}

High-resolution imaging of soft and hard tissues at once and in $3 D$, please!

\section{Philipp Schneider}

Bioengineering Science Research Group, Faculty of Engineering and the Environment, University of Southampton, Southampton, UK

Significant progress has been made over the last two decades to scrutinize bone porosity and particularly intracortical microstructure down to a cellular level, with important implications for our understanding of bone physiology, mechanics, and mechanobiology. However, this focus on bone porosity can divert the attention from the important interplay between angiogenesis and osteogenesis and the role of the vasculature for bone health and bone diseases for instance. On this account, recent advancements in high-resolution 3D X-ray and electron microscopy imaging will be presented here, which can be used to assess hard and soft tissues simultaneously, including mineralized bone tissue and soft tissues. The presentation will also shortly touch on our recent development of a novel micro-computed tomographic system that is optimized for soft tissues, an area traditionally considered suboptimal or unsuitable for X-ray imaging.

\section{IS8}

\section{The functions of the osteocyte change with age}

\section{Lynda Bonewald}

Indiana Center for Musculoskeletal Health, Anatomy, and Cell Biology and Orthopaedic Surgery, Indiana University School of Medicine, Indianapolis, IN, USA

It is not only osteoblasts and osteoclasts that are critical for bone remodeling but also the osteocyte, a central regulator of the activity of these two bone cell types in the growing, mature, and aging skeleton. The osteocyte is not only the bone mechanosensor but also a hormonally responsive cell that translates these two types of stimuli into signals that synchronize osteoblast and osteoclast activity. This synchronization is accomplished through both positive and negative signals sent by the osteocyte. For example, the osteocyte can send negative signals to osteoblasts such as sclerostin and DKK1 and positive signals of bone formation such as prostaglandin and wnts. Osteocytes can produce both M-CSF and RANKL for osteoclast formation. With menopause and aging, the osteocyte takes on more of its role as a negative regulator of skeletal mass. This long-lived cell becomes senescent, a state that is more likely to support resorption. The osteocyte can also function similarly to the osteoclast by producing factors such as TRAP and Cathepsin $\mathrm{K}$ to remove their perilacunar matrix under calcium-demanding conditions. Under physiological conditions, this perilacunar matrix is replaced, whereas under pathological conditions, this process continues and becomes detrimental with regard to bone mass. The osteocyte is also an endocrine cell that produces factors that target distant organs such as the kidney through factors such as FGF23. FGF23 is regulated by Phex and Dmp1, early osteocyte factors, and MEPE, a protein made by the mature osteocyte. Muscle function and myogenesis can be increased by factors made by osteocytes such as Wnt3a and prostaglandin; however, with aging, osteocytes produce unknown factors that decrease muscle mass.

deally, manipulating the osteocyte so that it functions on the side of bone formation and/or maintenance of bone mass is a major goal. Recently, it has also been described that muscle factors such as irisin or BAIBA can also retain bone mass under conditions of unloading. Mechanical loading (exercise) maintains and extends healthy functions of osteocytes to retain bone mass. Loading of bone and factors made by contracted muscle may be a new avenue for designing therapeutics to maintain bone health.

\section{IS9}

Epigenetics of early life origins of disease

\section{Karen Lillycrop}


Institute of Developmental Sciences, Biological Sciences, University of Southampton, Southampton, UK

There is now substantial evidence from both human epidemiological studies and animal models that an adverse intrauterine environment induced by a variety of environmental and maternal factors such as diet, body composition, or endocrine factors can induce a phenotype in the offspring that is characterized by an increased risk of developing chronic noncommunicable diseases in later life. The mechanism by which cues about nutrient availability in the postnatal environment are transmitted to the fetus and the process by which different, stable phenotypes are induced are beginning to be understood and involve the epigenetic regulation of specific genes. Epigenetic processes induce heritable change in gene expression without altering gene sequence. The major epigenetic mechanisms include DNA methylation, histone modification and non-coding RNAs. The epigenetic changes induced in response to nutritional cues from the mother may allow the fetus to adjust its developmental program in order to be better adapted to the future environment, while inappropriate adaptations may predispose an individual to increased risk of a range of non-communicable diseases.

This talk will describe how both maternal and paternal diet can influence the health of the child through the altered epigenetic regulation of genes, how epigenetic changes in early life may be used as predictive markers of future disease risk, and how nutritional interventions in postnatal life may be able to reverse the epigenetic and phenotypic changes induced by an adverse early life environment.

\section{IS10}

\section{Global perspective on early life determinants of non-communicable disease}

\section{Mark Hanson ${ }^{1,2}$ \\ ${ }^{1}$ Institute of Developmental Sciences, University of Southampton, Southampton, UK \\ ${ }^{2}$ NIHR Southampton Biomedical Research Centre, University of Southampton and University Hospital Southampton NHS Trust, Southampton, UK}

Non-communicable diseases (NCDs) pose an increasing threat to global health and economic sustainability in both high- and low-income countries, accounting for $>70 \%$ of deaths globally. Fixed genetic variation accounts for only a small fraction of inherited NCD risk, and adult lifestyle interventions have had disappointing impact. Risk of NCDs is set partly during early life, when environmental influences including mother's (and to an extent father's) diet, body composition, exposure to stress and smoking or unhealthy alcohol intake affect development of the fetus and newborn, conditioning its responses to later environmental challenges such as an obesogenic lifestyle. If the cues that the baby detects are inaccurate, eg, as a result of unbalanced maternal diet or because lifestyle transition occurs between generations through migration or rapid economic development, its responses are mismatched to later environmental challenges, leading to greater NCD risk. Additionally, parents with obesity and NCDs such as diabetes can pass risk to their children, perpetuating the cycle across multiple generations.
Gestational diabetes is also increasing and carries risk of later type 2 diabetes for both mother and child. Epidemiological, clinical, and basic science research has indicated underlying mechanisms, many of which involve epigenetic processes. These can serve as early markers of later risk, may be reversible, and could be used to monitor efficacy of interventions. Adopting a life course approach to the primary prevention of NCDs is now essential, starting in the preconception period by promoting healthy diets, body composition, and behavior among adolescents and young adults, not only for their later health but also for that of the next generation. While the problem of NCDs is global, sustainable solutions will have to be country-and culturally - specific. The life course approach to NCD prevention is now included in UN and WHO initiatives.

\section{IS11}

\section{Inflammation and musculoskeletal ageing}

\section{Janet Lord}

Institute of Inflammation and Ageing, University of Birmingham, Birmingham, UK

\section{IS12}

\section{Neglected patients at high risk of hip fractures}

\section{Steven R Cummings}

San Francisco Coordinating Center, University of California, San Francisco, CA, USA

Based on recent systematic reviews and meta-analyses, the risk of hip fracture is increased by at least twofold in patients with Parkinson's disease, recent stroke, dementia, HIV, heart failure, previously hospitalized with chronic obstructive pulmonary disease (COPD), end-stage renal disease, and type 1 (but not type 2) diabetes. Patients over age 65 years with Parkinson's disease also have more than a $10 \%$ per year risk of clinical fractures. In the United States, there are more patients with a high risk of fracture due to these conditions than patients with "osteoporosis" defined by a hip $T$-score $\leq-2.5$. Besides the increased risk of fracture, patients with these comorbidities generally have poorer outcomes after hip fracture. For this reason, the "treatment threshold" probability of fracture that warrants drug treatment should be substantially lower for patients with these conditions than for other patients. Nevertheless, relatively few patients with these conditions receive treatment; for example, in the US, fewer than $5 \%$ of older patients with Parkinson's disease have received a prescription for an approved treatment for osteoporosis and only half of those received 2 prescriptions. Specialists who care for them have little experience with assessments and drug treatments to prevent fracture, and extra screening is barrier to many patients with comorbid conditions. Because these patients have generally been excluded from fracture prevention trials, there is no evidence that improving BMD would reduce their fracture risk. Compared with standard practice, treating older patients with these comorbid conditions without individual risk screening would reach many more patients at high risk of disabling fractures and treatment with zoledronic acid would overcome their poor persistence. 


\section{IS13}

Osteo-angiogenic coupling in skeletal development, bone homeostasis, and fracture repair

\section{Christa Maes}

Laboratory of Skeletal Cell Biology and Physiology (SCEBP), Skeletal Biology and Engineering Research Center (SBE), Department of Development and Regeneration, KU Leuven, Leuven, Belgium

A critical step during endochondral ossification in bone development as well as homeostatic bone renewal and fracture repair is the invasion of avascular cartilage by blood vessels and the recruitment of immature osteoprogenitor cells (OPCs) to sites of bone formation. The invasion of OPCs and blood vessels occurs in a tightly spatio-temporally synchronized manner, with some OPCs being wrapped around blood vessels as pericytes. This temporal and local synchronization of osteogenesis and angiogenesis is referred to as osteo-angiogenic coupling. Interestingly, many perivascular subpopulations in the bone marrow environment are thought to function as "reserve" skeletal progenitors or stem cells and as regulators of the local bone marrow microenvironment, including supporting roles for hematopoietic stem cell maintenance and functioning.

Previous findings underline the significance of osteo-angiogenic coupling for skeletal physiology and hematopoietic integrity, and suggest an intense crosstalk between osteogenic cells and endothelial cells (ECs) that is conceivably involved in skeletal health and disease and could bear significant therapeutic value. However, the molecular mechanisms mediating the osteoangiogenic crosstalk and the recruitment of OPCs to the vessels are far from completely characterized to date.

Researchers at the KU Leuven SCEBP Lab aim to gain novel insights in the molecular control of skeletal cell functioning, with a focus on mesenchymal progenitors and osteoblast lineage cells and their interplay with the skeletal vasculature. The lab's research program is directed at understanding the mechanisms underlying bone formation in development, adult homeostasis, and fracture healing, but also in the significance of osteogenic cell biology in the broader physiological context of the organism, including hematopoiesis and global energy metabolism. Prime working models are genetically modified mice, including conditional and inducible knockout mice, in combination with fluorescent reporters and lineage tracing strategies.

\section{Oral Presentation Abstracts}

\section{OC1}

Social deprivation predicts adverse health outcomes after hospital admission with hip fracture in England

\section{Arti Gauvri Bhimjiyani, ${ }^{1}$ Jenny Neuburger, ${ }^{2}$ Yoav Ben- Shlomo, ${ }^{3}$ and Celia L Gregson ${ }^{1}$}

\footnotetext{
${ }^{1}$ Musculoskeletal Research Unit, Translational Health Sciences, Bristol Medical School, University of Bristol, Bristol, UK

${ }^{2}$ Department of Health Services Research and Policy, London School of Hygiene and Tropical Medicine, London, UK

${ }^{3}$ Population Health Sciences, Bristol Medical School, University of Bristol, Bristol, UK
}

Social deprivation predicts a range of adverse health outcomes; however, its impact on outcomes after a hip fracture is not established. We examined the effect of area-level social deprivation on outcomes after hospital admission with a hip fracture in England.

We used English Hospital Episodes Statistics linked by NHS Digital to the National Hip Fracture Database (04/2011-03/ 2015) and Office for National Statistics mortality database to identify patients admitted with hip fracture, aged $60+$ years. Deprivation was measured using quintiles of the Index of Multiple Deprivation (Q1 = least deprived; $Q 5=$ most deprived). Associations between deprivation and 30-day mortality and emergency 30-day readmission are described using odds ratios (ORs); logistic regression was used to adjust for age. Mean length of stay (LOS) in NHS acute and rehabilitation hospitals ("superspell") was calculated; the association between deprivation and mean LOS was estimated using linear regression. Total NHS bed occupancy within 1 year post-fracture was also calculated.

We identified 218,907 hospital admissions with an index hip fracture over 4 years. Median [IQR] was age 84 [78-89] years; $72.6 \%$ female. Overall 30-day mortality was $7.8 \%(n=17,072 /$ 218,907). Among survivors, median superspell was 16 (10-28) days, and $12.4 \%$ were readmitted within 30 days $(n=19,497 /$ 157,303), median 1-year bed occupancy 21 (11-41) days.

Greater deprivation was associated with higher 30-day mortality (Q5: 8.4\% $[n=3,229 / 38,434]$ versus Q1: 7.2\% $[n=3143 / 43,866])$, age-adjusted OR 1.30 (95\% Cl [1.24, 1.37], $p<0.001)$, equating to on average 2697 excess deaths per year occurring among those who are deprived (quintiles $2-5$ versus 1 ). Among survivors, ageadjusted mean superspell was longer in the most deprived versus least deprived quintile (Q5: 16.2 [15.3-17.1] days, Q1: 14.2 [13.6-14.7], $p<0.001)$. The 30-day readmission rate was higher in those most deprived $13.7 \%(\mathrm{Q} 5: n=3708 / 27,001)$ compared with those least deprived $11.4 \%$ (Q1: $n=3693 / 32,353)$, ageadjusted OR 1.27 [1.21, 1.34], $p<0.001$. A similar trend was observed when assessing mean 1-year NHS bed occupancy in the 71.9\% who survive to 1 year (Q5: 24.7 [23.2-26.2] days; Q1: 20.8 [19.9-21.7], $p<0.001$ ).

Greater deprivation is associated with reduced 30-day survival and among those who do survive, longer hospital stays and a greater need to be readmitted to hospital once discharged. The extent to which the configuration of English hospital services, rather than patient case-mix, explains these apparent health inequalities remains to be determined.

\section{OC2}

Tram2 is a novel genetic determinant of bone mass and strength

\section{Victoria D Leitch, ${ }^{1}$ Penny Sparkes, ${ }^{1}$ John G Logan, ${ }^{1}$ Agnes Swiatkowska, ${ }^{2}$ Peter I Croucher, ${ }^{3}$ Christopher J Lelliott, ${ }^{2}$ Graham R Williams, ${ }^{1}$ and JH Duncan Bassett ${ }^{1}$}

\author{
${ }^{1}$ Molecular Endocrinology Laboratory, Department of Medicine, \\ Imperial College London, London, UK \\ ${ }^{2}$ Mouse Pipelines, Wellcome Trust Sanger Institute, Cambridge, UK \\ ${ }^{3}$ Bone Biology Division, Garvan Institute of Medical Research, \\ Sydney, Australia
}

Osteoporosis is the commonest skeletal disorder, affecting millions and costing billions of pounds annually. Bone mineral 
density is highly heritable, but only $12 \%$ of the phenotype variance is currently accounted for. Treatments reduce fracture risk by only $50 \%$, and there is urgent need to define new pathways that regulate bone turnover and strength.

We hypothesized that rapid-throughput phenotyping of knockout mice would identify novel susceptibility alleles for bone and mineral disorders and provide in vivo models to elucidate their molecular basis.

Translocation-associated membrane protein-2 knockout mice $\left(\right.$ Tram $\left.^{--}\right)$were identified in this screen with reduced body weight, deafness, and spontaneous fractures, despite normal serum biochemistry.

Detailed analysis (X-ray microradiography, micro-CT, backscattered-electron scanning-electron microscopy, biomechanical testing, $n=6$ per sex, per genotype) demonstrated short stature $(p<0.001$, ANOVA), grossly reduced bone mineral content and mineralization ( $p<0.001$, Kolmogorov-Smirnov test), profoundly reduced cortical ( $p<0.001$, ANOVA) and trabecular bone mass ( $p<0.001$, ANOVA), and decreased bone strength and stiffness ( $p<0.001$, ANOVA) in Tram $2^{-/}$mice. Tram $2^{-/-}$ primary osteoblasts had reduced proliferation ( $p<0.001, t$ test) and mineralization, while osteoclasts had more nuclei $(p<$ $0.001, t$ test) and increased resorption ( $p<0.01, t$ test) compared with wild type.

Tram2 lies downstream of BMP/Runx2 in osteoblasts and is associated with fracture in genomewide association studies. Tram2 is a component of the translocon responsible for the folding of type 1 collagen; however, we detected no abnormalities of type 1 collagen structure by electron microscopy or protein amount by Western blot in Tram $2^{-/}$mice. These data demonstrate that Tram2 is required for normal bone mineralization, structure, and strength. The abnormal skeletal phenotype in $\mathrm{Tram}^{-1-}$ mice likely results from impaired bone formation during growth, together with uncoupling of adult bone turnover, resulting in severe bone loss. Elucidation of the cellular and molecular mechanisms underlying this gross skeletal phenotype may identify novel tractable therapeutic targets for prevention and treatment of osteoporosis.

\section{OC3}

Novel combination therapy with zoledronic acid and antiTGF $\beta$ repairs established osteolytic lesions in preclinical models of multiple myeloma

\section{Julia Paton-Hough, ${ }^{1}$ Simon Tazzyman, ${ }^{1}$ Holly Evans, ${ }^{1}$ Darren Lath, ${ }^{1}$ Jenny Down, ${ }^{1}$ Alanna Green, ${ }^{1}$ John Snowden, ${ }^{2}$ Michelle Lawson, ${ }^{1}$ and Andrew Chantry ${ }^{1,2}$}

\section{${ }^{1}$ Department of Oncology and Metabolism, University of Sheffield, Sheffield, UK \\ ${ }^{2}$ Department of Haematology, Sheffield Teaching Hospitals NHS Foundation Trust, Royal Hallamshire Hospital, Sheffield, UK}

Multiple myeloma is a plasma cell malignancy, which develops in the bone marrow and frequently leads to severe bone destruction. Current anti-resorptive therapies to treat the bone disease do little to repair damaged bone; therefore, new treatment strategies incorporating bone anabolic therapies are urgently required. We hypothesized that combination therapy using the standard-of-care anti-resorptive zoledronic acid (Zol) with a bone anabolic (anti-TGF $\beta / 1 D 11$ ) would be more effective at treating myeloma-induced bone disease than Zol therapy alone. JJN3 myeloma-bearing mice treated with combined Zol and 1 D11 resulted in a $48 \%$ increase $(p \leq 0.001)$ in trabecular bone volume fraction compared with Zol alone and a $65 \%$ ( $p \leq$ 0.0001) increase compared with 1D11 alone. The most significant finding was the substantial repair of U266-induced bone lesions with combination therapy, which resulted in a significant reduction in lesion area compared with vehicle ( $p \leq$ 0.01 ) or Zol alone $(p \leq 0.01)$. These results reveal a novel finding and demonstrate that combined anti-resorptive and bone anabolic therapy are significantly more effective at treating established myeloma-induced bone disease than Zol alone. This is a highly translational strategy that could significantly improve bone outcomes and quality of life in myeloma patients.

\section{OC4}

Systematic fracture risk screening using FRAX results in greater use of, and adherence to, anti-osteoporosis medications: the UK SCOOP Trial

\section{Camille Parsons, ${ }^{1}$ Nick Harvey, ${ }^{1,2}$ Lee Shepstone, ${ }^{3}$ John A Kanis, ${ }^{4,5}$ Elizabeth Lenaghan, ${ }^{3}$ Shane Clarke, ${ }^{6}$ Richard Fordham, ${ }^{3}$ Neil Gittoes, ${ }^{7}$ Ian Harvey, ${ }^{3}$ Richard Holland, ${ }^{3}$ Ali Heawood, ${ }^{6}$ Niamh Redmond, ${ }^{6}$ Amanda Howe, ${ }^{3}$ Tarnya Marshall, ${ }^{3}$ Tim Peters, ${ }^{6}$ David Torgerson, ${ }^{8}$ Terence W $\mathrm{O}^{\prime}$ Neill, ${ }^{9}$ Eugene McCloskey, ${ }^{4,10}$ Cyrus Cooper, ${ }^{1,2,11}$ and the SCOOP Trial Group}

${ }^{1}$ MRC Lifecourse Epidemiology Unit, University of Southampton, Southampton, UK

${ }^{2}$ NIHR Southampton Nutrition Biomedical Research Centre, University of Southampton and University Hospital Southampton NHS Foundation Trust, Southampton, UK

${ }^{3}$ University of East Anglia, Norwich, UK

${ }^{4}$ Centre for Metabolic Bone Diseases, University of Sheffield, Sheffield, UK

${ }^{5}$ Institute for Health and Ageing, Catholic University of Australia, Melbourne, Australia

${ }^{6}$ University of Bristol, Bristol, UK

${ }^{7}$ Queen Elizabeth Hospital, Birmingham, UK

${ }^{8}$ University of York, York, UK

${ }^{9}$ University of Manchester, Manchester, UK

${ }^{10}$ Mellanby Centre for Bone Research, Centre for Integrated research in Musculoskeletal Ageing, University of Sheffield, Sheffield, UK, and Sheffield Teaching Hospitals Foundation Trust, Sheffield, UK

${ }^{11}$ NIHR Oxford Biomedical Research Centre, University of Oxford, Oxford, UK

Objectives Despite the availability of validated risk assessment tools, such as FRAX, and highly cost-effective medications, there is little evidence that osteoporosis screening leads to increased use of anti-osteoporosis medications (AOM) and subsequent adherence. We investigated the effect of a population-based, primary care-led fracture risk screening intervention, on initiation of AOM, and subsequent adherence.

Methods The Screening for Prevention of Fracture in Older Women (SCOOP) study is a UK multi-center RCT of screening for osteoporotic fracture risk. A total of 12,483 women (70-85 years) were randomized to either assessment using the FRAX tool \pm DXA BMD, with medication recommended for those found at high fracture risk, or to usual NHS care. Self-reported AOM use was obtained by postal questionnaire at $6,12,24,36,48$, and 
60 months. Ordered logistic regression was used to explore baseline determinants of adherence (defined as \% study visits at which participants reported taking AOM, after a positive report at the index visit).

Results The mean (SD) age of participants was 75.6 (4.2) years, with 6233 randomized to screening and 6250 to the control group. At 6 months, $73.8 \%$ of participants classified at high fracture risk were taking AOM (and $12.3 \%$ of screening participants), compared with $2 \%$ of all participants in the control group. A total of $37.1 \%$ in the screening group on treatment at 6 months were still treated at 60 months; the respective figure for the control group was $21.6 \%$. For those participants commenced on treatment later during follow-up, rates of adherence at 60 months became increasingly similar between intervention and control participants (eg, $70.3 \%$ versus $68.1 \%$, respectively, at 60 months for treatment initiated at 48 months). Older age was associated with lower adherence $(\mathrm{OR}=0.96[95 \% \mathrm{Cl} 0.93,0.98], p=0.001)$ and history of parental hip fracture with greater adherence $(\mathrm{OR}=1.68[95 \% \mathrm{Cl} 1.28$, 2.20], $p<0.001$ ).

Conclusion Systematic fracture risk screening using FRAX leads to markedly greater use of AOM and greater adherence, in women at high fracture risk, compared with usual care. These findings inform public health strategies aimed at reduction of fragility fractures.

\section{OC7}

Age at onset of walking in infancy is associated with hip shape in early old age

\section{Alex Ireland, ${ }^{1}$ Stella Muthuri, ${ }^{2}$ Fiona Saunders, ${ }^{3}$ Anastasia Pavlova, ${ }^{3}$ Rebecca Hardy, ${ }^{2}$ Kathryn Martin, ${ }^{3}$ Rebecca Barr, ${ }^{3,4}$ Judith Adams, ${ }^{5}$ Diana Kuh, ${ }^{2}$ Richard Aspden, ${ }^{3}$ Jennifer Gregory, $^{3}$ and Rachel Cooper ${ }^{2}$}

\author{
${ }^{1}$ School of Healthcare Science, Manchester Metropolitan \\ University, Manchester, UK \\ ${ }^{2}$ MRC Unit for Lifelong Health and Ageing, University College \\ London, London, UK \\ ${ }^{3}$ Aberdeen Centre for Arthritis and Musculoskeletal Health, \\ University of Aberdeen, Aberdeen, UK \\ ${ }^{4}$ Medicines Monitoring Unit (MEMO), University of Dundee, \\ Dundee, UK \\ ${ }^{5}$ Manchester Academic Health Science Centre and Radiology, \\ Manchester Royal Infirmary, Manchester, UK
}

Bone shape and structure adapt in response to the large muscle and reaction forces they experience during everyday movements. The largest forces that routinely act upon the skeleton occur during bipedal movements such as walking, where wholebody mass must be accelerated against gravity on a single limb. Onset of independent walking at $\sim 12$ months represents the first postnatal exposure to these locomotory forces. Previous work has shown bone size and mass advantages in earlierwalking toddlers, which are also evident in adolescents and older adults. However, associations between early life loading and joint shape have not been explored.

We therefore examined associations between walking age and hip shape in later life in 1423 individuals (740 women) from the MRC National Survey of Health and Development (NSHD), a nationally representative British birth cohort. Walking age in months was obtained from maternal interview at age 2 years.
Ten modes of variation in hip shape (HM1-HM10), described by statistical shape models, were ascertained from dual-energy $X$ ray absorptiometry (DXA) images taken at ages 60-64 years. In analyses adjusted for sex, height, body composition, and socioeconomic position, earlier walking age was associated with higher HM1 and HM7 scores. Earlier walking was also associated with lower HM2 scores in women only (sex interaction $p=0.088$ ) and lower HM4 scores in men only (sex interaction $p=0.013$ ). Taken together, in earlier walkers, these modes describe a larger (HM4), flatter $(H M 2,4)$ femoral head, a wider $(H M 1,4,7)$, flatter (HM7) femoral neck, smaller neck-shaft angle (HM1,4), external rotation (HM2,7) and osteophytes (HM1).

These results suggest that age at onset of walking in infancy is associated with differences in hip shape in older age. In combination, hip modes suggest features of an osteoarthriticlike phenotype in earlier walkers. Unlike results of previous studies of walking age and bone mass, associations in this study were not affected by adjustment for lean mass. This suggests that associations may relate directly to skeletal loading in early life where joint shape changes rapidly, as opposed to later childhood and adolescence when skeletal changes primarily relate to increased size.

\section{OC6}

A 9-month jumping intervention to improve bone acquisition in adolescent male athletes: the PRO-BONE randomized controlled trial

\section{Dimitris Vlachopoulos, ${ }^{1}$ Alan Barker, ${ }^{1}$ Esther Ubago- Guisado, $^{1,2}$ Craig Williams, ${ }^{1}$ and Luis Gracia-Marco ${ }^{1,2}$}

${ }^{1}$ Sport and Health Sciences, University of Exeter, Exeter, UK ${ }^{2}$ Physical Education and Sport Sciences, University of Granada, Granada, Spain

Objectives: Participation in different loading sports during growth can have different effects on bone status and development. However, there is no evidence how to improve bone acquisition in adolescent athletes involved in weightbearing and non-weight bearing sports. The purpose was to examine the effect of a 9-month jumping intervention program on bone mass, geometry, and microarchitecture in adolescent males participating in weight-bearing (football [FOO]) and non-weight bearing (swimming [SWI] and cycling [CYC]) sports.

Methods: Ninety-three adolescent males (13-15 years) were included. Sport groups were randomized to intervention and sport (INT-SWI $=19$, INT-FOO $=15$, INT-CYC $=14$ ) or sport only $(\mathrm{CON}-\mathrm{SWI}=18, \mathrm{CON}-\mathrm{FOO}=15, \mathrm{CON}-\mathrm{CYC}=12)$. The intervention comprised a progressive jumping program of 3 levels ( 3 months each) using weighted vests (Level $1=20$ jumps, 0 kg, 3 sets/day, 3 times/week; Level $2=20$ jumps, 2 kg, 4 sets/day, 3 times/week; Level $3=20$ jumps, 5 kg, 4 sets/day, 4 times/week). Dual-energy X-ray absorptiometry (DXA) assessed bone mineral content (BMC); hip structural analysis (HSA) assessed crosssectional area (CSA), cross-sectional moment of inertia (CSMI) and section modulus; trabecular bone score (TBS) assessed bone microarchitecture; and quantitative ultrasound assessed bone stiffness before and after the intervention. One-way analysis of covariance compared the bone gains after controlling for preintervention bone, change in lean mass, and post maturity status. Significance was set at $p<0.05$. 
Results: INT-CYC gained significantly $(p<0.05)$ higher total body less head BMC (5.0\%), lumbar spine BMC (4.6\%), femoral neck BMC (9.8\%), and bone stiffness (12.3\%) than CON-CYC. INTCYC gained significantly higher CSA (11.0\%), CSMI (10.1\%), and TBS (4.4\%) outcomes than CON-CYC. INT-SWI gained significantly $(p<0.05)$ higher femoral neck BMC (6.0\%), legs BMC (4.2\%), and bone stiffness (12.7\%) than CON-SWI. INT-SWI gained significantly $(p<0.05)$ higher CSMI outcomes $(10.9 \%)$ than CONSWI. There were no significant $(p>0.05)$ differences between INT-FOO and CON-FOO for any bone outcomes (0.9-3.9\%).

Conclusion: The present 9-month jumping intervention improved bone outcomes in non-weight-bearing sports, such as swimming and cycling, but not in a weight-bearing sport, such as football.

\section{OC7}

Plasma $\beta$-C-terminal telopeptide of type I collagen (CTX) in pregnancy is influenced by cholecalciferol supplementation and is associated with maternal bone health: findings from the MAVIDOS trial

\section{Elizabeth M Curtis, ${ }^{1}$ Kate Maslin, ${ }^{1}$ Stefania D'Angelo, ${ }^{1}$ Rebecca J Moon, ${ }^{1,2}$ Sarah R Crozier, ${ }^{1}$ Fatma Gossiel, ${ }^{3}$ Nicholas J Bishop, ${ }^{4}$ Stephen Kennedy, ${ }^{5}$ Aris T Papageorghiou, ${ }^{5}$ Robert Fraser, ${ }^{6}$ Saurabh V Gandhi, ${ }^{6}$ Ann Prentice, ${ }^{7}$ Hazel M Inskip, ${ }^{1,9}$ Keith M Godfrey, ${ }^{1,9}$ Inez Schoenmakers, ${ }^{7,8}$ M Kassim Javaid, ${ }^{10}$ Richard Eastell, ${ }^{3}$ Cyrus Cooper, ${ }^{1,9,10}$ Nicholas C Harvey, ${ }^{1,9}$ and the MAVIDOS Trial Group}

\footnotetext{
${ }^{1}$ MRC Lifecourse Epidemiology Unit, University of Southampton, Southampton, UK

${ }^{2}$ Paediatric Endocrinology, University Hospitals Southampton NHS Foundation Trust, Southampton, UK

${ }^{3}$ Academic Unit of Bone Metabolism, University of Sheffield, Sheffield, UK

${ }^{4}$ Academic Unit of Child Health, Sheffield Children's Hospital, University of Sheffield, Sheffield, UK

${ }^{5}$ Nuffield Department of Women's \& Reproductive Health, John Radcliffe Hospital, University of Oxford, Oxford, UK

${ }^{6}$ Department of Obstetrics and Gynaecology, Sheffield Hospitals NHS Trust, University of Sheffield, Sheffield, UK

${ }^{7}$ MRC Human Nutrition Research, Elsie Widdowson Laboratory, Cambridge, UK

${ }^{8}$ Department of Medicine, Faculty of Medicine and Health Sciences, University of East Anglia, Norwich, UK

${ }^{9}$ NIHR Southampton Nutrition Biomedical Research Centre, University of Southampton and University Hospital Southampton NHS Foundation Trust, Southampton, UK

${ }^{10}$ National Institute for Health Research (NIHR) Oxford Biomedical Research Centre, University of Oxford, UK
}

Objectives: In a randomized trial, we investigated associations between 1) gestational vitamin $D$ supplementation and maternal/umbilical cord $\beta$-C-terminal telopeptide of type I collagen (CTX) concentrations; 2 ) maternal gestational $25(\mathrm{OH})$ vitamin D status and maternal/umbilical cord CTX; and 3) CTX and maternal postnatal bone indices.

Methods: MAVIDOS is a randomized, double-blind, placebocontrolled trial of $1000 \mathrm{IU} /$ day cholecalciferol from 14 weeks' gestation to birth. Maternal plasma CTX (Roche ECLIA), plasma 25(OH)D (Diasorin Liaison), health, and anthropometry were assessed at 14 and 34 weeks' gestation. Newborn CTX was measured in umbilical cord plasma. Maternal bone indices were assessed by DXA within 2 postnatal weeks.

Results: 636 women (314 placebo, 322 cholecalciferol) were included, with a measure of CTX in early pregnancy (14 weeks' gestation, EP), late pregnancy (34 weeks' gestation, LP), or in umbilical cord blood at birth (cCTX).

CTX increased overall from EP to LP (median 219.4 to $437.4 \mathrm{pg} /$ $\mathrm{mL}$; $p$-diff $<0.0001)$; EP and LP values were correlated in both the placebo $(r=0.31)$ and treatment $(r=0.45)$ groups (both $p<$ 0.0001). LPCTX was higher in the placebo than treatment group (median 445.3 versus $420.0 \mathrm{pg} / \mathrm{mL} ; p=0.06$ ).

In the treatment group only, CCTX was negatively associated with increasing $[25(\mathrm{OH}) \mathrm{D}]$ from EP to LP $(\beta=-0.002(95 \% \mathrm{Cl}$ $-0.004,-0.001) \mathrm{pg} / \mathrm{mL} / \mathrm{nmol} / \mathrm{L}, p=0.008)$. Associations persisted after adjustment for birth season.

In general, LPCTX, but not EPCTX, was negatively associated with maternal whole-body bone mineral content $(\beta / 10 \%$ CTX increase $-1.70 \mathrm{~g}[-2.80,-0.60], p<0.01)$, bone area $\left(-1.05 \mathrm{~cm}^{2}\right.$ $[-1.67,-0.43], p<0.001)$ and bone mineral density $(-0.0003 \mathrm{~g} /$ $\left.\mathrm{cm}^{2}[-0.0006,-0.0001], p=0.04\right)$.

Conclusion: In this randomized trial, MAVIDOS, gestational vitamin $D$ supplementation was associated with lower maternal late pregnancy CTX. The associations between CTX and maternal and cord 25(OH)D, and maternal bone indices, inform mechanisms linking pregnancy vitamin D status to bone health.

\section{OC8}

Relationships between the hypothalamic-pituitary-adrenal axis and bone microarchitecture: findings from the Hertfordshire Cohort Study

\section{Nicholas Fuggle, ${ }^{1}$ Leo Westbury, ${ }^{1}$ Holly Syddall, ${ }^{1}$ Kate Ward, ${ }^{1}$ Niharika Duggal, ${ }^{2}$ Janet Lord, ${ }^{2}$ Cyrus Cooper, ${ }^{1,3}$ and Elaine Dennison ${ }^{1}$}

\author{
${ }^{1}$ MRC Lifecourse Epidemiology Unit, University of Southampton, \\ Southampton, UK \\ ${ }^{2}$ MRC-ARUK Centre for Musculoskeletal Ageing, Institute of \\ Inflammation and Ageing, University of Birmingham, Birmingham, \\ $U K$ \\ ${ }^{3}$ NIHR Musculoskeletal Biomedical Research Unit, University of \\ Oxford, Oxford, UK
}

Objectives: Circulating cortisol levels increase slightly with age, but levels of dehydroepiandrosterone sulphate (DHEAS) decline rapidly from the age of 30 years. As cortisol is primarily catabolic and DHEAS is converted to the anabolic steroid DHEA, this increase in the cortisol/DHEAS ratio with age may have significant implications for bone health. The objective of this study was to examine the longitudinal associations between morning cortisol and DHEAS, in relation to bone microarchitecture outcomes in a population-based UK cohort of older adults.

Methods: Analyses were based on a sample of 339 communitydwelling older persons (aged 59-70 years at baseline) from the Hertfordshire Cohort Study. At baseline, serum cortisol and DHEAS levels were measured by ELISA (IBL International, Hamburg, Germany). High-resolution peripheral quantitative computed tomography (HRpQCT) imaging of the radius and tibia (XtremeCT, voxel size $82 \mu \mathrm{m}$ ) was performed at follow-up (median follow-up time: 11.6 years [IQR 11.0-12.4]). Sexadjusted linear regression was used to examine the relationships 
between cortisol, DHEAS, and cortisol/DHEAS ratio, in relation to bone microarchitecture outcomes, with and without adjustment for anthropometric and lifestyle factors.

Results: Higher cortisol was associated with higher radial cortical porosity and pore volume in fully adjusted analyses only $(p<0.03)$. Higher DHEAS was associated with higher tibial cortical BMD and trabecular thickness and lower tibial total and trabecular area in sex-adjusted analyses $(p<0.04)$; the association regarding trabecular thickness remained significant in fully adjusted analyses. In sex- and fully adjusted analyses, higher values of the cortisol/DHEAS ratios were associated ( $p<$ 0.04) with lower values of the following tibial parameters: cortical BMD, total volumetric BMD, and trabecular thickness.

Conclusion: Our work identifies the HPA axis as a contributor to bone microarchitecture and musculoskeletal phenotype and suggests a particular role for DHEAS. These findings build on previous work performed using DXA and further dissect the relationship between the HPA axis and bone parameters. This may have future therapeutic implications; however, further research is required to investigate these associations.

\section{OC9}

Repurposing glutamate receptor antagonists for the prevention of post-traumatic osteoarthritis

\section{Cleo Bonnet, Sophie Gilbert, Emily Eccles, Kingsley Norman, and Deborah Mason}

\section{School of Biosciences, Cardiff University, Cardiff, UK}

Objectives: We previously found that AMPA and kainate glutamate receptors (GluRs) localize to osteoarthritic bone, cartilage, and synovium. NBQX (AMPA/kainate GluR antagonist) reduced knee swelling, gait abnormalities, and joint destruction in inflammatory and post-traumatic osteoarthritis rodent models. Since NBQX is not approved for human use, we have tested 4 AMPA/kainate GluR antagonists (A-D, passed Phase 1 clinical trials) and compared these to current approved treatments (hyaluronic acid [HA], steroid intra-articular injections) in a mouse ACL-rupture model.

Methods: For ACL rupture, a $12 \mathrm{~N}$ load (ElectroForce 3200, BOSE) is applied to anesthetized C57Bl6 mice. A $10-\mu \mathrm{L}$ intra-articular injection of drug $\mathrm{A}(n=4), \mathrm{B}(n=3), \mathrm{C}(n=3), \mathrm{D}(n=4), \mathrm{HA}(n=8$, Durolane, $8 \mathrm{mg} / \mathrm{kg}$ ) or steroid ( $n=7$, Depo-medrone, $10 \mathrm{mg} / \mathrm{kg}$ ) was administered immediately after rupture; additional mice received vehicle ( $100 \mathrm{mM} \mathrm{NaOH}$ : drug $\mathrm{A}, n=4$; DMSO: drugs $\mathrm{B}-$ $\mathrm{D}, n=4$; saline: $\mathrm{HA}$ /steroid, $n=7$ ). Over 21 days, lameness and knee swelling were recorded. On day 21, animals were culled and knees scored for degradation (OARSI).

Results: By day 2, drug A reduced swelling ( $~ 50 \%)$ and did not differ significantly from day 0 measurements, whereas swelling remained increased until day 7 in vehicle $(p<0.01$, generallinear model [GLM]). Drug $C$ reduced swelling to day 0 levels by day 2; D ( $p<0.05, \mathrm{GLM})$ and vehicle $(p<0.001, \mathrm{GLM})$ were increased until day 7. Drug $B$ did not reduce swelling until day 14. On day 1 , steroid significantly reduced swelling ( $\sim 50 \%)$ compared with vehicle ( $p<0.001, \mathrm{GLM})$ and HA $(p<0.05, \mathrm{GLM})$; this was short-lived with no reductions from day 2 . HA had no effect. Lameness scores were reduced by drugs $A-D$, with significant reductions compared with vehicle on day 3 by drug $B$ ( $p<0.001, \mathrm{GLM}), \mathrm{C}(p<0.05, \mathrm{GLM})$, and D ( $p<0.05, \mathrm{GLM})$. HA and steroid had no effect. Drug $A$ reduced degradation score at day 21 ( 40\%); steroid increased degradation $(\sim 50 \%, p<0.05$, one-way ANOVA).

Conclusion: This study shows that AMPA/kainate GluR antagonists, already approved for human use, relieve inflammation, lameness, and joint degradation in post-traumatic osteoarthritis. GluR antagonists exceeded anti-nociceptive, anti-inflammatory, and anti-degradation effects of $\mathrm{HA}$ and steroids. Surprisingly, HA had no effects, whereas steroids substantially increased degeneration.

\section{OC10}

MMnet, a macrophage multinucleation network, enriched in genes controlling osteoclast function

\section{Marie Pereira, ${ }^{1,2}$ Jeong-Hun Ko, ${ }^{1,2}$ John Logan, ${ }^{2}$ Enrico Petretto, ${ }^{2,4}$ JH Duncan Bassett, ${ }^{2}$ Graham R Williams, ${ }^{2}$ and Jacques Behmoaras ${ }^{1}$}

\author{
${ }^{1}$ Centre for Complement and Inflammation Research, Imperial \\ College, London, UK \\ ${ }^{2}$ Molecular Endocrinology Laboratory, Imperial College, London, UK \\ ${ }^{3}$ Wellcome Trust Sanger Institute, Hinxton, UK \\ ${ }^{4}$ Duke-NUS Medical School, Singapore, Republic of Singapore
}

Macrophages fuse to form osteoclasts in bone or multinucleated giant cells (MGCs) in granulomatous disorders. MGC and osteoclast fusion display a common molecular signature, suggesting shared genetic determinants. We used a systems genetics approach in rat macrophages that undergo spontaneous formation to identify the genetic determinants of multinucleation. By mapping genomewide expression quantitative trait loci, we identified a co-expression network of 143 trans-regulated genes associated with macrophage multinucleation (MMnet) that was enriched for known osteoclast genes.

To validate the role of MMnet genes in macrophage fusion and identify novel determinants of bone mass and strength, we have developed rapid-throughput skeletal phenotyping of knockout mice generated by the International Knockout Mouse Consortium (IKMC). Knockout mice with deletions of 12 MMnet genes have been analyzed in this pipeline (App/2 ${ }^{-/ \text {, }}$

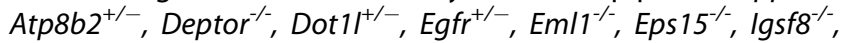
Pik3 $\mathrm{Cb}^{-/}, \mathrm{Rnf125^{-- }}$, Slc1 $\mathrm{a} 4^{-/}, \mathrm{Tgfb} 1 \mathrm{i1}^{-/}$) using quantitative X-ray microradiography, micro-computed tomography, and biomechanical testing ( $n=2-6$ per line). Outliers were identified with phenotype parameters lying $>2.0$ standard deviations from the reference mean derived from $>300$ wild-type mice of identical age, sex, and genetic background. RNA interference was used to knock down each of the 12 genes in primary human macrophages, and the effects on osteoclastogenesis, fusion, and bone resorption were determined $(n=4)$. Dcstamp, an established master regulator of multinucleation, was included as a positive control.

Eight of the 12 knockout lines had outlier skeletal phenotypes affecting linear growth, bone mass, or strength. This represented a highly significant enrichment compared with unselected knockout lines (chi-square test, $p<0.0001$ ). For example, deletion of $P i k 3 c b$ (PI3-kinase catalytic subunit-beta) resulted in a $68 \%$ increase in trabecular BV/TV. Furthermore, knockdown of $P i k 3 c b$ in human macrophages in vitro reduced the number of multinucleated osteoclasts ( $4+$ nuclei) by $83 \%$ and decreased hydroxyapatite resorption by $89 \%$. Similar analysis of all 11 
Mmnet genes revealed a strong positive correlation between multinucleation and resorption.

These data demonstrate that MMnet genes regulate macrophage multinucleation and bone resorption in vitro, together with skeletal development and adult bone mass in vivo, and could allow identification of novel regulators of macrophage fusion.

\section{OC11}

Bromodomain inhibitors are potent epigenetic regulators of catabolic gene expression in human osteoarthritic chondrocytes

\section{Maria C de Andres, ${ }^{1}$ Namrata Madhusudan, ${ }^{1}$ Chas Bountra, ${ }^{2}$ Udo Oppermann, ${ }^{2}$ and Richard OC Oreffo ${ }^{1}$}

\author{
${ }^{1}$ Bone and Joint Research Group, University of Southampton, \\ Southampton, UK \\ ${ }^{2}$ Structural Genomic Consortium, University of Oxford, Oxford, UK
}

Objectives: Osteoarthritis (OA) is a complex disease of the articulation characterized by joint pain, functional limitation, and reduced quality of life. An essential feature of the underlying pathogenesis is an imbalance of anabolic and catabolic activity leading to progressive loss and destruction of extracellular matrix of articular cartilage. There is increasing evidence to support the role of DNA methylation in the pathogenesis of OA. However, few studies have investigated the effect of other epigenetic modifications in this debilitating disease. The current study has analyzed the effects of three novel epigenetic modifiers: two bromodomains inhibitors (JQ-1, PFI-1) and a histone methyltransferase inhibitor (SGC707) on the catabolic gene expression involved in the pathogenesis of OA.

Methods: Chondrocytes extracted from OA femoral heads $(n=6)$ were cultured and subsequently incubated with increasing concentrations of the compounds. Interleukin 1beta (IL-1 $\beta$ ) plus oncostatin M (OSM) alone or in combination with JQ-1, PFI-1, or SGC707 or media alone (control) were added to the incubated samples. After 6 and 24 hours, levels of iNOS, COX2, IL8, IL1B, matrix metalloproteinase-13 (MMP13), RUNX2, and $C O L 9 A 1$ gene expression were measured using qRT-PCR and expressed relative to GAPDH.

Results: The bromodomain inhibitors JQ-1 (100 and $800 \mathrm{nM}$ ) and PFI-1 $(0.5$ and $5 \mu \mathrm{M})$ were able to suppress not only the expression of all the catabolic cytokines after co-stimulation with IL-1 $\beta+$ OSM but also the basal expression of OA chondrocytes without stimulation. Critically, preliminary results with JQ1 and PFI-1 in OA chondrocytes show that this BET inhibitors are able to decrease catabolic gene expression (iNOS, COX2, IL8, IL1B, and MMP13), RUNX2 expression was also suppressed, and no effect was observed on the expression of the anabolic chondrocytic gene COL9A1. Interestingly, the histone methyltransferase inhibitor SGC707 (0.1 and $1 \mu \mathrm{M})$ did not induce any reduction in the expression of all the catabolic genes.

Conclusion: This study demonstrates that inhibition of bromodomains by epigenetic modifiers modulates the expression of catabolic genes in OA chondrocytes. These results further substantiate the role of epigenetics in OA with implications for therapeutic intervention and our understanding of OA pathophysiology.

\section{OC12}

Application of 3D-printed patient-specific skeletal implants augmented with autologous skeletal stem cells

\section{Vitali Goriainov, ${ }^{1}$ Josephine McEwan, ${ }^{1}$ Kate White, ${ }^{1}$ Richard Oreffo, ${ }^{1}$ and Douglas Dunlop ${ }^{2}$}

\author{
${ }^{1}$ Bone and Joint Research Group, University of Southampton, \\ Southampton, UK \\ ${ }^{2}$ Trauma and Orthopaedics Department, University Hospital \\ Southampton, NHS Foundation Trust, Southampton, UK
}

Objectives: Patient-specific 3D-printed hip replacements are used in complex surgical reconstruction in the presence of skeletal tissue loss. We present a clinical case series in which custom-designed 3D-printed implants have been augmented intra-operatively with autologous skeletal stem stems (SSCs) to enhance bone-implant osseointegration. In vitro studies were performed to characterize the behavior of SSCs on the customdesigned implant surface.

Methods: Clinical: Patients unsuitable for routine revision hip surgery (ie, salvage cases) were offered surgical intervention using custom-designed implants seeded with autologous SSCs. Primary clinical outcome measures were Oxford Hip Score (OHS) pre- and post-operatively; secondary measures were complications and implant failure.

In vitro: A STRO-1-positive cell population was isolated from the nucleated cell fraction of human bone marrow aspirate. Cells were seeded onto metal substrate blocks that corresponded to the custom-designed implant (porous), a standard implant (planar), and tissue culture plastic (TCP) (control). Substrates were cultured for 21 days, and live/dead cell viability was performed at days 7 and 14. At day 21, the cells were trypsinized and RNA extracted for molecular qPCR analysis of osteogenic expression.

Results: All patients had an improvement between pre- and post-operative OHS $(p<0.001)$. There were no surgical complications and, critically, to date, no implant failures. Postoperative CT scans demonstrate encouraging new bone formation at the bone-implant interface. Live/dead cell viability assay demonstrated live cells on the planar and porous substrates and TCP. Day $21 \mathrm{qPCR}$ revealed statistically significant enhancement in expression of osteogenic genes (alkaline phosphatase, collagen type 1, osteopontin, and osteocalcin) on the porous substrate.

Conclusion: Clinical results demonstrate that custom-designed implants seeded with autologous SSCs offer a viable treatment option in salvage cases with substantial skeletal tissue loss, with excellent early to mid-term outcomes. The in vitro studies demonstrate enhanced osteogenic gene expression on the porous substrate, which suggests that the customdesigned implant surface supports osseointegration when combined with SSCs. Improved osseointegration should improve implant survivorship, and longer-term follow-up is required to evaluate this.

\section{OC13}

Bovine lactoferrin promotes bone regeneration

\section{Jillian Cornish}

Medicine, University of Auckland, Auckland, New Zealand 
Objectives: Lactoferrin is a multifunctional glycoprotein with therapeutic potential in the field of bone regenerative medicine/ tissue engineering. We have previously shown that lactoferrin in vitro and in vivo has potent stimulating effects in osteoblast proliferation and differentiation, is an anti-apoptotic agent, and has inhibitory effects on osteoclastogenesis. Following on from this earlier research, we have assessed the efficacy of local application of lactoferrin on bone regeneration.

Methods: Using a preclinical critical-sized calvarial defect model in the rat, bovine lactoferrin $(10 \mu \mathrm{g})$ impregnated in a collagen gel was grafted into animals that were euthanized at 4 or 12 weeks post-operatively. The calvariae were excised and evaluated with micro-CT (Skyscan 1172) followed by histology. Results: The bone volume fraction (BV/TV) was higher in lactoferrin-treated animals (group 1) at both time points when compared with control animals that received collagen or had an empty defect (groups 2 and 3). The results were $8.6 \pm 1.4 \%$, $16.5 \pm 0.6 \%$, and $24.27 \pm 2.6 \%$, respectively, at 4 weeks $(p<0.05)$ and $13.6 \pm 1.5 \%, 21.9 \pm 1.2 \%$, and $29.3 \pm 0.8 \%$, respectively, at 12 weeks $(p<0.05)$. Histological analysis revealed that the newly formed bone within the calvarial defects of all groups was a mixture of woven and lamellar bone, with more bone in the group treated with lactoferrin at both time points.

Conclusion: A release of lactoferrin from the scaffold significantly increased bone regeneration. The profound effect of lactoferrin in innovative bone regeneration studies has therapeutic potential to improve the poor clinical outcomes associated with bony non-union. Non-united bone fractures occur at an alarming rate due to increases in osteoporosis in the aging population. Furthermore, lactoferrin has anti-microbial properties and modulates the immune response, which are very positive properties for bone healing.

We acknowledge the source of funding from Health Research Council of New Zealand, New Zealand Orthopaedic Association Research Foundation, and the New Zealand Wishbone Trust.

\section{OC14}

Increased sclerostin expression following OB-derived VEGF deletion

Alice Goring, ${ }^{1}$ Alan Boyde, ${ }^{2}$ Eric Hesse, ${ }^{3}$ Napoleone Ferrara, ${ }^{4}$ Bjorn Olsen, ${ }^{5}$ Andrew Pitsilides, ${ }^{6}$ Richard Oreffo, ${ }^{7}$ Philipp Schneider, ${ }^{8}$ and Claire Clarkin ${ }^{1}$

${ }^{1}$ Biological Sciences, University of Southampton, Southampton, UK

${ }^{2}$ Institute of Dentistry, Queen Mary University of London, London, UK

${ }^{3}$ Heisenberg Group for Experimental Trauma Surgery, University of Hamburg, Hamburg, Germany

${ }^{4}$ Genetech, San Francisco, CA, USA

${ }^{5}$ Department of Cell Biology, Harvard School of Dental Medicine, Boston, MA, USA

${ }^{6}$ Comparative Biomedical Sciences, Royal Veterinary College, London, UK

${ }^{7}$ Faculty of Medicine, University of Southampton, Southampton, UK

${ }^{8}$ Faculty of Engineering and the Environment, University of Southampton, Southampton, UK

Objectives: Vascular Endothelial Growth Factor (VEGF) is required for coupling of osteogenesis and angiogenesis. Intracrine VEGF signaling has been previously described to control the differentiation of osteoblasts $(\mathrm{OBs}){ }_{i}^{(1)}$ however, there is also evidence that VEGF utilizes paracrine signaling pathways to regulate osteogenesis via vascular endothelial cells (ECs). The aim of this study was to delete VEGF in mature osteocalcinexpressing cells in vivo and in vitro and to assess effects on vascular and bone phenotype.

Methods: For OB-specific VEGF knockout (OBVEGFKO), adult male mice carrying floxed alleles of VEGF and expressing Cre recombinase under the control of the osteocalcin promotor were used. Cortical bone phenotype was investigated by histology, immunohistochemistry, and backscattered electron scanning electron microscopy (BSE-SEM). In vitro, long bonederived OBs were isolated from Vegf flox/flox mice and VEGF deleted using Adenovirus-Cre-GFP with Adenovirus-GFP (WT) used as control. Conditioned media from VEGFKO and WT OBs were added to bone marrow ECs for gene expression analyses. Results: Increased blood vessels (BV) were present in the bone cortex of OBVEGFKO tibia (\% BV area; WT $0.54 \pm 0.29$, OBVEGFKO $3.6 \pm 1.28, \quad p=0.05)$ with individual blood vessel canals surrounded by unmineralized osteoid. BSE-SEM further identified deficiencies in bone mineralization in the absence of OBVEGF, with immature woven bone prevalent and an abundance of unmineralized osteoid, which contained large, irregularly spaced osteocyte lacunae. To investigate the potential for ECs in driving this bone phenotype, ECs were treated with conditioned media collected from OBs with VEGF deleted and WT controls. Conditioned media from WT OBs (which contained VEGF) suppressed sclerostin mRNA expression in ECs (-2.14-fold); however, in response to OBVEGF deletion, ECs increased sclerostin mRNA expression $(+3.21$-fold). These in vitro findings were validated by immunohistochemical labelling, which revealed elevated sclerostin levels localized to CD31+ECs in OBVEGFKO bone.

Conclusion: Deletion of OB-derived VEGF increases endothelial cell expression of sclerostin, which could contribute to the poorly mineralized phenotype observed in OBVEGF KO animals. Funded by Arthritis Research UK.

\section{Reference}

1. Liu Y, Berendsen AD, Jia $S$, et al. Intracellular VEGF regulates the balance between osteoblast and adipocyte differentiation. J Clin Invest. 2012;122(9):3101-13.

\section{OC15}

Serum citrate: a novel biomarker of bone resorption?

April Hartley, ${ }^{1,2}$ Lavinia Paternoster, ${ }^{2}$ John Kemp, ${ }^{3}$ David Evans, ${ }^{3}$ Jonathan Tang, ${ }^{4}$ William Fraser, ${ }^{4}$ Jon H Tobias, ${ }^{1}$ and Celia L Gregson ${ }^{1}$

\footnotetext{
${ }^{1}$ Musculoskeletal Research Unit, Translational Health Sciences, Bristol Medical School, University of Bristol, Bristol, UK

${ }^{2}$ Integrative Epidemiology Unit, Population Health Sciences, Bristol Medical School, University of Bristol, Bristol, UK

${ }^{3}$ The University of Queensland Diamantina Institute, Translational Research Institute, Queensland, Australia

${ }^{4}$ Department of Medicine, Norwich Medical School, University of East Anglia, Norwich, UK
}

Objectives: As well as contributing to osteoporosis pathogenesis, increased bone turnover may exert metabolic consequences. To characterize these, we examined relationships between bone turnover markers (BTMs) and the output of a metabolomics screen, in a population enriched by High Bone Mass (HBM). 
Methods: We analyzed 320 adults including 198 with HBM (BMD $Z$-score $>3.2$ ) and 122 unaffected relatives/spouses. BTMs $\beta-C$ terminal telopeptide of type I collagen (CTX), procollagen type 1 amino-terminal propeptide (P1NP), and osteocalcin were assessed by electrochemiluminescence immunoassays. Metabolic traits, including lipids, apolipoproteins, glycolysis-related metabolites, amino acids, and a biomarker of inflammation were measured using high-throughput nuclear magnetic resonance spectroscopy. Associations of BTMs with metabolic traits were assessed using generalized estimating equation linear regression to account for correlation within families. We adjusted for age, sex, menopause, height, weight, alcohol consumption, lipid-lowering medication, and bisphosphonate use.

Results: The mean age was 59.1 years (SD 15.0); 64\% were female. In unadjusted analysis, out of 21 metabolic traits analyzed, serum citrate was positively associated with all BTMs. After adjustment, CTX and osteocalcin remained positively associated with citrate: $\beta=0.20$ (95\% Cl 0.07, 0.33), $p=0.002$ and $\beta=0.14(0.03,0.24), p=0.011$, respectively ( $\beta=$ SD increase in citrate for an SD increase in BTM). Evidence for a positive association with P1NP was weaker after adjustment $(\beta=0.10$ $[-0.02,0.22], p=0.089)$. When all BTMs were added to a multivariable model, only CTX remained independently associated with citrate $(0.22[0.01,0.42], p=0.042)$. Subsequently, we sought replication of the latter association in 1617 adolescents from the Avon Longitudinal Study of Parents and Children, in whom CTX and metabolic traits had been measured (mean age 15.5 years [SD 0.3]; $57 \%$ female). We detected a strong positive correlation between citrate and CTX $\left(r=0.38, p=2.4 \times 10^{-59}\right)$. Conclusion: Bone resorption, reflected by CTX, is positively related to serum citrate levels. Taken with previous reports that citrate is found at high concentrations in bone, where it has been suggested to play a role in bridging mineral platelets, serum citrate may represent a novel resorption marker, differing from CTX by reflecting breakdown of bone mineral as opposed to collagen.

\section{OC16}

Women at high risk of hip fracture based on FRAX respond to appropriate osteoporosis management: analysis from the SCOOP study of population screening

\section{Eugene McCloskey, ${ }^{1,6}$ Nicolas Harvey, ${ }^{2}$ Helena Johansson, ${ }^{3,5}$ Lee Shepstone, ${ }^{4}$ Elizabeth Lenaghan, ${ }^{4}$ Cyrus Cooper, ${ }^{2}$ John Kanis, $^{3,5}$ and SCOOP Study Team

\author{
${ }^{1}$ Oncology \& Metabolism, University of Sheffield, Sheffield, UK \\ ${ }^{2}$ Medical Research Council Lifecourse Epidemiology Unit, University \\ of Southampton, Southampton, UK \\ ${ }^{3}$ Centre for Metabolic Bone Diseases, University of Sheffield, \\ Sheffield, UK \\ ${ }^{4}$ School of Medicine, University of East Anglia, Norwich, UK \\ ${ }^{5}$ Australian Catholic University, Melbourne, Australia \\ ${ }^{6}$ Centre for Integrated Research in Musculoskeletal Ageing, \\ University of Sheffield, Sheffield, UK
}

Objectives: Targeting of treatment in interventional studies to reduce osteoporotic fractures has usually been based on low $\mathrm{BMD}$ and/or prior fracture rather than the absolute risk of fracture. The recently completed primary-care-based SCOOP screening study targeted treatment to those at highest hip fracture risk using FRAX. We wished to examine the impact of the screening intervention on hip fracture risk according to baseline FRAX hip fracture probability.

Methods: The SCOOP study comprised a two-arm randomized controlled trial in women aged 70 to 85 years comparing a screening program versus usual management. In the screening arm, treatment was recommended in women identified to be at high risk of hip fracture by FRAX (including BMD). Agedependent intervention thresholds were used ranging from $5.24 \%$ in 70 - to 74 -year-olds to $8.99 \%$ hip fracture probability in 85-year-olds.

Results: Of 12,483 eligible participants, 6233 women were randomized to screening, with treatment recommended in 898 (14.4\%); amongst these, anti-osteoporosis medication had been prescribed in over $70 \%$ by 6 months. In the screening arm, the number of incident hip fractures was lower than that in the control arm (218 versus 164 ), reflecting a $28 \%$ reduction in hip fracture risk (hazard ratio $=0.72,95 \% \mathrm{Cl} 0.59$ to $0.89, p=0.002$ ). As treatment was targeted to those at highest risk, the effect on hip fracture increased significantly with baseline FRAX hip fracture probability ( $p=0.021$ for interaction); for example, at the 10th percentile of baseline FRAX hip probability (2.6\%), hip fractures were not significantly reduced $(\mathrm{HR}=0.93,95 \% \mathrm{Cl} 0.71$ to 1.23$)$, but at the 90 th percentile $(16.6 \%)$, there was a $33 \%$ reduction ( $\mathrm{HR}=0.67,95 \% \mathrm{Cl} 0.53$ to 0.84$)$.

Conclusion: Women identified to be at high fracture risk based on FRAX hip fracture probability are responsive to appropriate osteoporosis management. Community-based screening using the FRAX tool is feasible and effective.

\section{OC17}

Osteoblast-specific Enpp1 deficiency engenders increased bone mass and insulin resistance

Fiona Roberts, ${ }^{1}$ Nabil Rashdan, ${ }^{1}$ Isabel Orriss, ${ }^{2}$ Katherine Staines, ${ }^{3}$ Elspeth Milne, ${ }^{1}$ Faisal Ahmed, ${ }^{4}$ Nicholas Morton, ${ }^{5}$ Colin Farquharson, ${ }^{1}$ and Vicky MacRae ${ }^{1}$

${ }^{1}$ The Roslin Institute and Royal (Dick) School of Veterinary Studies, University of Edinburgh, Edinburgh, UK

${ }^{2}$ Department of Comparative Biomedical Sciences, Royal Veterinary College, London, UK

${ }^{3}$ Biomedical Science Research Group, School of Applied Sciences, Edinburgh Napier University, Edinburgh, UK

${ }^{4}$ Developmental Endocrinology Research Group, School of Medicine, Dentistry, and Nursing, University of Glasgow, Glasgow, UK ${ }^{5}$ Centre for Cardiovascular Science, Queen's Medical Research Institute, University of Edinburgh, Edinburgh, UK

The principal bone mineralization inhibitor ectonucleotide pyrophosphatase/phosphodiesterase-1 (NPP1) has recently emerged as a pathogenic factor predisposing to insulin resistance and type 2 diabetes mellitus. We have previously demonstrated that Enpp $1^{-/-}$mice exhibit pronounced resistance to obesity and insulin resistance after chronic high-fat diet (HFD) feeding. Here, we determined the impact of osteoblast-specific Enpp1 ablation on HFD-induced obesity.

Mice lacking NPP1 specifically in osteoblasts (Ob-KO) were generated by crossing Enpp $1^{\text {flox/flox }}$ and Osteocalcin-Cre mice. Male mice were reared on a control diet $(6.2 \%$ fat) or HFD (58\% fat) up to 16 weeks of age. Metabolic tests, micro-computed tomography of long bones, dissection, gait, and histological analysis were performed. 
Under control dietary conditions, Ob-KO mice exhibited increased bone mass, indicated by increased femoral bone volume/total volume $(17.50 \%$ versus $11.67 \% ; p<0.01)$ and reduced trabecular spacing $(0.187 \mathrm{~mm}$ versus $0.157 \mathrm{~mm} ; p<$ $0.01)$ at 6 weeks of age. Adult Ob-KO mice exhibit increased muscle mass (13.3\%; $p<0.05)$, likely resultant of increased gait instability (increased base of support [15\%; $p<0.05]$, girdle support [66\%; $p<0.05]$, initial [117\%; $p<0.05]$ and terminal [128\%; $p<0.05$ ] forelimb dual stance). Adult mice showed normal glucose and insulin tolerance tests (GTT/ITT) comparable between genotypes. No notable differences in body weight or gross anatomy were observed.

After chronic HFD challenge, no significant differences in body weight gain, muscle, or white fat mass were observed between genotypes. However, Ob-KO mice showed significantly increased liver (30.1\%; $p<0.05)$ and brown fat mass (22.2\%; $p<0.05)$. These Ob-KO mice showed decreased glucose and insulin tolerance $(p<$ 0.05 ) after GTT and ITT, respectively. No significant difference in pancreatic islet size or number between genotype were observed. These data indicate that the protection against diabetes noted in Enpp $1^{-/-}$mice is likely due to the actions of non-skeletal NPP1. Indeed, osteoblast-specific NPP1 deficiency drives an unexpected worsening of insulin sensitivity relative to control mice on HFD. Together, these data indicate that NPP1 inhibition at its major site of expression is metabolically detrimental. Further studies are required to establish the mechanisms through which NPP1 regulates its actions within and out with bone.

\section{OC18}

A new dual injury osteoarthritis model that combines cartilage damage and destabilization of the medial meniscus leads to accelerated osteophytogenesis

\author{
Kendal McCulloch, ${ }^{1}$ Carmen Huesa, ${ }^{1}$ Lynette Dunning, ${ }^{1}$ Rob \\ van't Hof, ${ }^{2}$ John Lockhart, ${ }^{1}$ and Carl Goodyear ${ }^{3}$ \\ ${ }^{1}$ Institute of Biomedical \& Environmental Health Research, \\ University of the West of Scotland, Paisley, UK \\ ${ }^{2}$ Institute of Ageing and Chronic Disease, University of Liverpool, \\ Liverpool, UK \\ ${ }^{3}$ Institute of Immunity, Infection \& Inflammation, University of \\ Glasgow, Glasgow, UK
}

Objectives: Osteoarthritis (OA) is synonymous with articular cartilage damage. However, in what way disease pathogenesis is initiated is unknown. It is presumed that it can be through an isolated injury or a combination of joint damage over time. Combined with the fact cartilage has poor regenerative capability, this represents a major clinical challenge. Several murine models are used to study OA, but none combine simultaneous cartilage injury with joint destabilization. The aim of this study was to investigate if combining cartilage damage and DMM accelerates the onset of OA-like symptoms.

Methods: OA was induced in mice via (a) transection of the medial meniscotibial ligament (DMM), (b) microblade scratches of articular cartilage (cartilage damage), or (c) combined DMM and cartilage scratch (DCS). Fourteen days post-surgery, dynamic weight bearing was assessed as an indirect measurement of pain, and micro-computed tomography $(\mu \mathrm{CT})$ was used to monitor bone changes.

Results: Osteophytes were present in all groups, but between groups there were significant differences in number, appearance, and bone volume. Osteophytes in the DCS model encompassed a larger area of subchondral bone compared with DMM or cartilage scratch models $(14.12 \pm 0.31$ versus $12.4 \pm 0.62,12.68 \pm 0.54, p<0.01$ respectively). In DMM, 6/8 mice developed protruding osteophytes $(1 \pm 0.8)$ with an arboreal-like structure. Osteophytes $(1.4 \pm 0.5)$ were present in all mice that underwent cartilage scratch surgery, with a larger, more calcified appearance. Importantly in the DCS model, all mice exhibited significantly larger, protruding, calcified osteophytes $(2.6 \pm 0.5 ; p<0.001)$. Moreover, mice in the DCS model demonstrated a significant increase in front paw load compared with the other models ( $p<0.05$ ). Notably, there is a significant and strong correlation between a change in weight bearing and both osteophyte number and osteophyte size $(R=0.7$, $p=0.003 ; \mathrm{R}=0.74, p=0.001$, respectively), suggesting an increase in OA-like pain.

Conclusion: Combining DMM with cartilage damage provides a robust and reproducible model for OA-associated osteophytogenesis. This model also incorporates OA/osteophyte-related pain, arguably one of the most problematic and physically limiting symptoms of OA.

\section{OC19}

Longitudinal survey of high-density mineralized protrusions: a potential novel imaging biomarker of osteoarthritis

\author{
Neil Peter Thomas, ${ }^{1}$ Nathan Jeffery, ${ }^{1}$ Lakshminarayan \\ Ranganath, ${ }^{1}$ Alan Boyde, ${ }^{2}$ and James Gallagher ${ }^{1}$ \\ ${ }^{1}$ Human Anatomy Resource Centre, University of Liverpool, \\ Liverpool, UK \\ ${ }^{2}$ Queen Mary University of London, London, UK
}

Microcracks in the subchondral bone plate and calcified cartilage can occur under normal loading conditions and are in some cases repaired with a high-density mineralized infill. This material may egress from the mineralizing front into hyaline articular cartilage (HAC) to form a high-density mineralized protrusion (HDMP). Historically overlooked, reports of HDMPs are increasing, having recently been confirmed in the ex vivo hips and knees of people with and without osteoarthritis (OA). This is the first longitudinal MRI study prospecting for HDMPs. Clinical scans were acquired from patients $(n=21)$ in the Osteoarthritis Initiative progression cohort. Sagitally acquired dual-echo steady-state MRI data for both knees were assessed for HDMPs at five annual visits. Incidence was characterized topographically according to six regions: medial femoral condyle (MFC), lateral femoral condyle (LFC), intercondylar femur (ICF), medial tibial condyle (MTC), lateral tibial condyle (LTC), and intercondylar tibia (ICT).

Seventeen of 21 patients possessed HDMPs in $\geq 1$ knee: 12 bilaterally and 5 unilaterally. Fifty-eight HDMPs were identified in total. Regional distribution in order of highest to lowest incidence was: ICF (36\%), LFC (25\%), LTC (23\%), MFC (12\%), ICT (2\%), and MTC (2\%). Some HDMPs were present in all scans. However, some appeared and/or disappeared during the study. Of those identified at baseline, the percentage present at each subsequent annual visit was $96,90,85$, and 81 , respectively. Changes in morphology over time were noted. For example, some appeared to have grown. Others fragmented, forming clusters of HDMP material within HAC. 
Incidence of HDMPs in regions of high stress and their potential to fragment may account for clustering and the presentation of HDMPs over time and is suggestive of a role in arthropathy. Abrasive HDMP particles would be detrimental to the biomechanical performance of surrounding tissues. Longevity of HDMPs indicates that this process may be gradual and is not assured. As they form before identification of symptomatic OA and are clinically detectable, HDMPs are a potential primary cause of joint destruction and should be considered a novel imaging biomarker for predicting some forms of $O A$.

\section{Late-Breaking Oral Presentation Abstracts}

\section{LB1}

Pregnancy-induced changes in bone microarchitecture and density at the tibia as determined by single-slice and highresolution peripheral quantitative computed tomography

\author{
Mícheál ó Breasail, ${ }^{1}$ Ann Prentice, ${ }^{1}$ and Kate Ward ${ }^{1,2}$ \\ ${ }^{1} M R C$ Elsie Widdowson Laboratory, MRC, Cambridge, UK \\ ${ }^{2} M R C$ Lifecourse Epidemiology Unit, MRC, Southampton, UK
}

Objectives: The newborn skeleton contains 25 to $30 \mathrm{~g}$ of calcium, although the source of this has not been fully qualified. Evidence from DXA studies before and shortly after pregnancy suggests that some of this calcium may originate from the maternal skeleton. The aim of this study was to use single-slice and high-resolution peripheral quantitative computed tomography ( $\mathrm{QQCT}, \mathrm{HRpQCT}$ ) to characterize whether pregnancyinduced changes occur in the appendicular skeleton.

Methods: Pregnant women were scanned twice, at 14 to 16 and 34 to 36 weeks' gestation. Premenopausal controls were also scanned approximately 20 weeks apart. pQCT scans (XCT 2000L) were obtained at the distal (4\%) and proximal $(14,38 \%)$ sites of the non-dominant tibia. HRpQCT (XtremeCTI) scans of the distal tibia were also obtained. Outcomes were: distal total and trabecular vBMD, total cross-sectional area (CSA), plus for HRpQCT distal cortical VBMD and thickness, trabecular number, and thickness; pQCT proximal outcomes were cortical vBMD, cortical CSA, cortical thickness, and total CSA. Linear regression models were fitted and the model of best fit selected; covariates were group, height, change in weight, baseline age, time between visits, parity, smoking history, oral contraceptive use, and group*time difference. Group differences are presented as mean (SE).

Results: Ninety (53 pregnant) women, mean (SD) age 35.4 (3.8) years, were recruited. No significant differences were found between groups at baseline except trabecular thickness, which was lower in the pregnant group $(p<0.05)$. At follow-up, pregnant women had significantly lower distal total VBMD $(-1.9$ [0.6]\%); proximal cortical VBMD (14\%-0.9 [0.2]\%), 38\%-0.5 [0.2]\%). HRpQCT distal total (-1.8 [0.5]\%) and cortical vBMD $(-1.8[0.5] \%)$ were lower in pregnant women, as were distal cortical and trabecular thickness $(-2.3[0.5] \%,-3.9[1.9] \%$, respectively). Trabecular number was greater (3.6 [1.8]\%) in pregnant women.

Conclusion: During pregnancy, bone mineral is mobilized predominantly from the cortical compartment of the distal tibia. These data also suggest there may be early pregnancy changes in trabecular bone, which have previously been reported in one study of bone histomorphology.
LB2

Zoledronate every 18 months for 6 years in osteopenic postmenopausal women: effects on fractures and nonskeletal endpoints

Ian Reid, Anne Horne, Borislav Mihov, Angela Stewart, Liz Garratt, Mark Bolland, Sonja Bastin, and Gregory Gamble

Medicine, University of Auckland, Auckland, New Zealand

Objectives: Bisphosphonates prevent fractures in patients with osteoporosis, but their efficacy in women with osteopenia is unknown. Most fractures in postmenopausal women occur in osteopenic individuals, so if pharmaceutical intervention is to impact significantly on total fracture numbers, therapies with efficacy in osteopenic postmenopausal women are needed.

Methods: We report a double-blind trial of 2000 osteopenic, postmenopausal women, randomly assigned to receive 4 infusions of either zoledronate $5 \mathrm{mg}$ or normal saline at 18 month intervals. Each was followed for 6 years. Monthly vitamin D supplements were provided but not calcium supplementation. Women were recruited using electoral rolls. Inclusion criteria were age $>65$ years, hip $T$-score between -1.0 and -2.5 . Exclusion criteria were: lumbar spine $T$-score $<-3.0$, eGFR $<30 \mathrm{~mL} /$ minute, major systemic disease, metabolic bone disease, or regular use of bone-active drugs in the previous year. The study has $80 \%$ power to detect a decrease in osteoporotic fractures of $30 \%$.

Results: At baseline, age was 71 (SD 5) years, BMI 27 (5), femoral neck $T$-score $-1.5(0.5)$, and $95 \%$ were white. Non-vertebral fractures (excluding skull, face, hands, and feet) occurred in 148 women in the placebo group and in 101 in the zoledronate group (ITT analysis, hazard ratio $=0.66[95 \% \mathrm{Cl} 0.51,0.85]$, $p=0.0014$, NNT $=22$ ). Height loss, a surrogate for vertebral fracture, was $9.3(8.7,9.9) \mathrm{mm}$ in the placebo group and $7.4(6.9$, $8.0) \mathrm{mm}$ in the zoledronate group $(p<0.0001)$. Odds ratio for prespecified adverse events were as follows: death, $0.65(0.40$, 1.046); myocardial infarction, $0.61(0.36,1.02)$; cancer 0.67 (0.50, $0.90)$. Odds ratio for breast cancer was $0.58(0.34,0.98)$.

Conclusion: These results suggest this less intensive zoledronate regimen is effective for fracture prevention in osteopenia and that it has beneficial effects on cancer risk and, possibly, mortality. These findings have the potential to substantially broaden the target population for pharmaceutical intervention to prevent fractures and suggest that zoledronate should be further explored for the prevention of cancer and vascular disease. Funded by the Health Research Council of New Zealand.

\section{Poster Presentation Abstracts}

\section{P1}

X-linked hypophosphatemia: burden of disease using United Kingdom primary care data
Muhammad Javaid, ${ }^{1}$ Antonella Delmestri, ${ }^{1}$ Nick Shaw, ${ }^{2}$ Daniel Prieto-Alhambra, ${ }^{2}$ Cyrus Cooper, ${ }^{1}$ and Rafael Pinedo- Villanueva ${ }^{1}$
${ }^{1}$ NDORMS, University of Oxford, Oxford, UK
${ }^{2}$ Paediatrics, Birmingham Women's Children's NHS Foundation Trust, Birmingham, UK


Objectives: To determine the prevalence of X-linked hypophosphatemia $(X L H)$ in children and adults in the UK to inform commissioning of novel therapeutic agents by regulatory authorities.

Materials and Methods: The Clinical Practice Research Datalink (CPRD) comprises the entire computerized medical records of a sample of patients attending general practitioners (GPs) in the UK. It currently contains information including Read codes, laboratory and prescription data on over 11 million patients (around $7 \%$ of the population) registered at over 600 general practices in the UK, that are representative of the population in terms of demographics such as age and sex. A list of Read codes was generated that included terms related to rickets, hypophosphatemia, and osteomalacia and cases extracted from 1995 to 2016. Two clinicians with experience in pediatric (NS) and adult XLH (MKJ) reviewed the potential cases using the Read codes, laboratory values for alkaline phosphate (maximum recorded levels) and serum phosphate (minimum recorded levels) and at least 1 year of prescriptions with 1-alfacalcidol or phosphate supplements. Cases were graded as highly likely, probable, possible, and unlikely. Only cases scored highly likely or probable by both readers were included.

Results: From the 522 potential cases, 34 (6.3\%) were scored as highly likely by both readers; 59 (11\%) were scored as either highly likely or probable by both readers (Kappa 0.7). Of the 59 cases, $63 \%$ were female. The prevalence of XLH was 0.65 cases per 100,000 .

Conclusion: This is the first estimation of the prevalence of XLH using routine primary care data, with approximately 430 cases in the UK. This is more aligned with data from Norway than South Denmark. A confirmatory verification questionnaire is being sent to all primary care providers for validation of all cases. Further work is needed to improve coding of XLH patients in primary care.

\section{P2}

Localization and partial deletion of the HGD gene in a new targeted model of alkaptonuria

\author{
Juliette Hughes, ${ }^{1}$ Peter Wilson, ${ }^{1}$ Ke Liu, ${ }^{1}$ Andrew Hughes, ${ }^{1,2}$ \\ Lakshminarayan Ranganath, ${ }^{1,2}$ James Gallagher, ${ }^{1}$ and \\ George Bou-Gharios ${ }^{1}$ \\ ${ }^{1}$ Institute of Ageing and Chronic Disease, University of Liverpool, \\ Liverpool, UK \\ ${ }^{2}$ Department of Clinical Chemistry, Royal Liverpool and \\ Broadgreen University Hospital Trust, Liverpool, UK
}

Objectives: A new AKU mouse model with targeted disruption of the homogentisate 1,2-dioxygenase (HGD) gene has been generated, replacing the mutagenesis model. To localize HGD expression in the adult mouse and determine when in embryonic development HGD is switched on, the knockout HGD tm1a allele and lacZ reporter construct situated within the trapping cassette were utilized. The effect of liver-specific deletion of HGD on the AKU phenotype was then investigated in the HGD tm1d mouse by removal of a floxed critical exon.

Methods: Fresh whole-mount adult HGD tm1a tissues and timemated embryos (E13.5 to E16.5) were lacZ stained. Frozen sections of liver and kidney were lacZ stained to look at histological localization of HGD.

Injection of plpC induces removal of a critical exon in HGD tm1d mice via MxCre recombinase. $\mathrm{HGD}$ tm1d MxCre +/- mice were injected twice with plpC (10 $\mu \mathrm{g} / \mathrm{g})$. HGD tm1d MxCre WT and HGD tm 1a mice were injected with plpC as WT and AKU controls. Homogentisic acid (HGA) levels were measured in plasma and urine taken pre- and post-injection to assess the phenotype. HGD expression was analyzed via qPCR.

Results: HGD is expressed in both the liver (cytoplasm of hepatocytes) and the proximal convoluted tubule cells of the kidney cortex. Embryo lacZ staining reveals that HGD expression begins at E14.5 in the liver.

Injection of plpC knocks down HGD expression in the liver but not the kidney of $\mathrm{MxCre}+/-$ mice. Plasma HGA increases from 0 to $47 \mu \mathrm{mol} / \mathrm{L}$ in the $\mathrm{plpC}$-injected $\mathrm{MxCre}+/-$ mice. Plasma HGA in HGD tm $1 \mathrm{a}$ and MxCre WT mice was $59 \mu \mathrm{mol} / \mathrm{L}$ and $0 \mu \mathrm{mol} / \mathrm{L}$, respectively. Urinary HGA increases in $\mathrm{MxCre}+/-$ mice from 22 to $8621 \mu \mathrm{mol} / \mathrm{L}$ but not to the level of HGD tm1a mice $(127,354 \mu \mathrm{mol} / \mathrm{L})$.

Conclusion: HGD is expressed in the liver and kidney, first being expressed at E14.5. MxCre recombinase has been successfully used to knock down HGD in the liver of MxCre +/- mice. The HGA levels in these mice increases in the plasma, suggesting that kidney HGD expression alone is not sufficient to rescue the AKU phenotype.

\section{P3}

A review of clinical, radiological, and treatment features from 18 patients with SAPHO (synovitis, acne, pustulosis, hyperosteosis, and osteitis) syndrome and CRMO (chronic recurrent multifocal osteomyelitis) at Addenbrooke's Hospital

\section{Jagtar Singh Nijjar, ${ }^{1}$ Jessica Padley, ${ }^{2}$ and Ken Poole ${ }^{1}$ \\ ${ }^{1}$ Department of Medicine, University of Cambridge, Cambridge, UK ${ }^{2}$ School of Clinical Medicine, University of Cambridge, Cambridge, UK}

Objectives: Rare diseases affect 1 in 10 patients, but despite this, there is a lack of research and evidence in these conditions. SAPHO affects 1 in 10,000 people with CRMO possibly being a pediatric form of the condition.

The aim of this study was to develop a data dictionary to facilitate collection of data into an eCRF (electronic case report form) and analyze the data collected if it was determined to be of sufficient quality ( $>50 \%$ complete from routine records).

Methods: A literature review was carried out to determine important clinical and radiological features of SAPHO and CRMO. These factors were then used to create a web-accessible eCRF database with military level security (www.castoredc.com). Patients were identified through the electronic health record system EPIC and a departmental database.

Results: Our cohort was equally matched in terms of sex and was mainly white in ethnicity. Seven patients were ever smokers, although 7 of 18 had no information recorded. Alcohol information was not routinely recorded in clinical practice. Median time from symptom onset to first rheumatology appointment was 4 years (IQR 3.25-5.5 years). Long bone and sacroiliac involvement was present in $50 \%$ of patients with 8 having anterior chest wall symptoms. In terms of skin and peripheral arthritis, 6 had both, 3 had arthritis only, 4 skin features only, and 5 had neither. Only 4 patients had an elevated CRP $(>10 \mathrm{mg} / \mathrm{dL}$ ) and 12 patients had an MRI ( $46 \%$ bone marrow edema, 23\% sclerosis). Nine patients had bisphosphonate, 6 disease-modifying drugs, and 1 anti-TNF. 
Conclusion: Clinical feature, radiological, and treatment data was well recorded in electronic record. This cohort was collected from a metabolic bone clinic and may account for the clinical feature and treatment skew. There is a significant delay from symptom onset to review with half of patients having active bone inflammation and a quarter showing sclerosis. This study clearly demonstrates the need for more research and evidence generation in these patients.

\section{P4}

Self-perception of fracture risk is associated with radial bone microarchitecture in the GLOW Study

\section{Leo Westbury, Anna Litwic, Kate Ward, Cyrus Cooper, and Elaine Dennison} MRC Lifecourse Epidemiology Unit, University of Southampton,
Southampton, UK

Objective: Self-perception of fracture risk (SPR) is associated with fracture risk, independent of FRAX. This study examined whether SPR is related to bone microarchitecture of the radius among participants of the Global Longitudinal study of Osteoporosis in Women (GLOW).

Methods: GLOW involves 723 physicians across 10 countries. Overall, 60,393 women aged 55 years and over completed annual questionnaires detailing medical history, comorbidities, and fractures; SPR was ascertained at the 5-year follow-up. UK participants underwent high-resolution peripheral quantitative computed tomography (HRpQCT) scans of the radius and dualenergy X-ray absorptiometry (DXA) scans of the hip (median follow-up time since SPR ascertainment: 2.8 years [interquartile range 2.3-3.7]).

Linear regression was used to examine the relationship between SPR in relation to HRpQCT parameters and hip areal bone mineral density (aBMD) with and without adjustment for anthropometric and lifestyle characteristics and use of antiosteoporotic medications. Associations additionally adjusted for hip aBMD were also explored.

Results: Analyses were restricted to 279 women with complete data on all predictors and outcomes of interest. Mean (SD) age at time of scan was 70.4 (5.2) years. Overall, 139 (49.8\%) women reported a similar self-perceived fracture risk compared with other women of the same age; 100 (35.8\%) reported a lower risk; and 40 (14.3\%) reported a higher risk.

At the radius, higher SPR was associated with lower trabecular number $(p<0.02)$ and higher trabecular separation $(p<0.03)$ in unadjusted and adjusted analyses and when additionally adjusted for hip aBMD. Higher SPR was associated with lower total area $(p<$ $0.04)$ in unadjusted analyses and associations were similar $(p<$ 0.06 ) in adjusted analyses and when additionally adjusted for hip aBMD. In unadjusted analyses only, higher SPR was associated $(p<$ 0.04 ) with lower cortical porosity and lower hip aBMD.

Conclusion: These results suggest that SPR is associated with bone microarchitecture and that some relationships remain after adjustment for hip aBMD. Further studies are required to delineate these associations.

\section{P5}

Cluster analysis of high-resolution peripheral quantitative computed tomography parameters including finite element analysis identifies bone phenotypes associated with higher rates of prevalent fracture

\section{Mark Edwards, Leo Westbury, Cyrus Cooper, Elaine Dennison, and Kate Ward}

MRC Lifecourse Epidemiology Unit, University of Southampton, Southampton, UK

Objectives: To identify clusters of bone microarchitecture and strength in older men and women and relate them to fracture prevalence and aBMD.

Methods: We studied 171 women and 188 men, aged 72.1 to 81.4 years, with $\mathrm{HRpQCT}$ (XtremeCT I) images of the distal radius. Standard image analyses were performed for assessment of macrostructure, regional densitometry, cortical porosity, trabecular microarchitecture, and finite element analysis. K-means partitioning cluster analysis was used to identify 5 clusters in women and 5 in men. Prevalent fracture rates and femoral neck aBMD were determined for each cluster.

Results: Fifty (29.2\%) women and 45 (23.9\%) men had fractures. Using microarchitecture and FEA parameters together, in women, there were two phenotypes with fracture risk greater than the reference (lowest fracture prevalence) cluster. Cluster $A$ contained 25 women ( $50 \%$ fractured) with Ct.Ar, Ct.Th, Ct.BMD, stiffness, and Young's modulus (YM) more than 1 SD below the sex-specific cohort mean, and \% Tb load (distal and proximal) more than 1 SD above. Cluster B (41 women, 38.5\% fractured) had a \% Tb load proximal more than 1 SD below the sex-specific cohort mean. Using FEA parameters alone, 3 clusters had significantly greater fracture risk. Two were similar to clusters $A$ and $B$ above. The third had stiffness, YM, cort stress, and cort strain more than 1 SD below the sex-specific cohort mean. In women, the reference cluster had higher aBMD compared with all other clusters $(p<0.05)$. Fracture rates did not differ significantly by cluster in men. However, similar phenotypes to clusters $A$ and $B$ were identified in the analyses.

Conclusion: We have demonstrated clusters with cortical and trabecular phenotypes associated with a higher proportion of fractures in women. In men, who had fewer fractures, significant differences in fracture rates were not identified despite similar phenotypes being identified.

\section{P6}

Severe lumbar spinal stenosis on MRI scans is related with heavy manual work: the Wakayama Spine Study

Yuyu Ishimoto, ${ }^{1}$ Cyrus Cooper, ${ }^{1,2}$ Georgia Ntani, ${ }^{1,2}$ Hiroshi Yamada, ${ }^{3}$ Hiroshi Hashizume, ${ }^{3}$ Shigeyuki Muraki, ${ }^{4}$ Sakae Tanaka, ${ }^{5}$ Munehito Yoshida, ${ }^{3}$ Noriko Yoshimura, ${ }^{6}$ and Karen Walker-Bone ${ }^{1,2}$

${ }^{1}$ MRC Lifecourse Epidemiology Unit, Southampton General Hospital, Southampton, UK

${ }^{2}$ Arthritis Research UK/MRC Centre for Musculoskeletal Work and Health, Southampton General Hospital, Southampton, UK

${ }^{3}$ Orthopedic Surgery, Wakayama Medical University, Wakayama, Japan

${ }^{4}$ Department of Preventive Medicine for Locomotive Organ Disorders, Faculty of Medicine, University of Tokyo, Tokyo, Japan ${ }^{5}$ Department of Orthopedic Surgery, Sensory and Motor System Medicine, Graduate School of Medicine, University of Tokyo, Tokyo, Japan

${ }^{6}$ Department of Preventive Medicine for Locomotive Organ Disorders, University of Tokyo, Tokyo, Japan 
Objective: Our objective is to investigate the contribution of occupations and physical workplace exposures to lumbar spinal stenosis (LSS) as investigated by magnetic resonance imaging (MRI) in a population-based cohort.

Materials and Methods: The Wakayama Spine Study recruited a population sample of adults from a range of different occupations. All participants underwent total spinal MRI in a mobile MRI unit (Excelart 1.5T; Toshiba, Tokyo, Japan). The severity of LSS, including central stenosis, was rated on a fourgrade scale such as none, mild, moderate, and severe by a spine surgeon. Using the job exposure information, occupational groups were classified in 6 groups: Clerical/Technical; Agricultural/Fishermen; Factory/Construction; Clinical/Housekeeper/ Shop assistants/Hairdressers/Dressmakers; Teachers and Others/No answer.

Results: Complete data were available for 722 participants (245 males, 477 females; mean age, 70.9 years; range, 53-93 years). In total, 239 (33\%) of subjects were defined with severe LSS and became the cases for these analyses and the remaining 483 patients were controls. We performed logistic regression analyses to estimate odds ratios (OR) and 95\% confidence intervals $(\mathrm{Cl})$ for associations between occupational group and severe LSS. Unadjusted analysis showed that Agricultural/Fishermen and Factory/Construction workers had a significantly higher risk of severe LSS when compared with Clerical/Technical experts (Agricultural/Fishermen: $\mathrm{OR}=1.99,95 \% \mathrm{Cl} 1.22-3.26$; Factory/Construction: $\mathrm{OR}=2.08,95 \% \mathrm{Cl} 1.09-3.96)$. The significant associations were lost after adjustment for age, sex, body mass index, smoking, and usual walking speed. However, stratification of the analyses by age ( $<75$ years and $\geq 75$ years) showed that the associations with severe LSS remained for Factory/ Construction workers aged $<75$ years even after adjustment for all the same confounders (Factory/Construction: OR $=3.97$, 95\% Cl 1.46-10.85).

Conclusion: Our data suggested a similar relationship between musculoskeletal degenerative disease and heavy manual work as well as other OA at several different anatomical sites. More research is required, but attention to ergonomic factors during working life may reduce the burden of long-term musculoskeletal symptoms, improving quality of life at older ages.

\section{P7}

Spontaneous atypical tibial and femoral fractures associated with alendronate then denosumab therapy in an adult patient with juvenile idiopathic arthritis: a case report

\author{
Juan Tan ${ }^{1}$ and Kenneth Poole ${ }^{2}$ \\ ${ }^{1}$ Foundation School, NHS Lothian, Edinburgh, UK \\ ${ }^{2}$ Metabolic Bone Disease Unit, University of Cambridge, \\ Cambridge, UK
}

Background: Atypical femoral fractures are a rare but serious complication of long-term anti-resorptive therapy. These fractures are defined according to specific criteria set out by the American Society for Bone and Mineral Research (ASBMR). Fractures with atypical features have been previously reported in other sites such as the tibia and pelvis but are much rarer. We describe a patient who had bilateral distal tibial and unilateral proximal femoral insufficiency fractures associated with alendronate then denosumab therapy for osteoporosis.
Case Report: Our patient is a 35-year-old white male who had received 6 years of alendronate therapy for glucocorticoidinduced osteoporosis secondary to severe juvenile (childhoodonset) and ongoing adult inflammatory arthritis. His clinician stopped the alendronate when he developed pain in his anterior left tibia with a fracture line (initially considered as "fragility") and commenced subcutaneous denosumab. Fifteen months after the fracture line appeared, he presented with spontaneous pain and tenderness in both tibias. Plain X-rays showed incomplete, non-displaced fractures in the anterior middlethird of both tibial diaphyses. "Atypical" features included a short oblique orientation; non-displacement, non-comminution; associated periosteal/endosteal reaction, and increased cortical thickening. Both tibias were openly reduced and internally fixed with plates and screws. Teriparatide was commenced. He later developed spontaneous complete bilateral tibial fractures adjacent to the plates and then a right atypical femoral fracture despite improvements in his bone density. His fractures were fixed surgically and he has since made good progress.

Discussion: Our case highlights that atypical fractures associated with antiresorptive therapy, known to occur in weightbearing long bones such as the femur, can also occur in the tibia. Risks of "oversuppression" fractures should be considered and a careful evaluation of any prevalent "stress" fractures should be borne in mind when electing to continue from one potent antiresorptive to another. Bony tenderness in a patient on long-term antiresorptive therapy should prompt urgent radiological investigation to exclude a fracture.

\section{P8}

\section{Osteoclasts are multinucleated cells that degrade cartilage as well as bone}

\section{Quitterie Larrouture, Helen Knowles, Sarah Snelling, and Nick Athanasou}

\section{Nuffield Department of Orthopaedics, Rheumatology and Musculoskeletal Sciences, University of Oxford, Oxford, UK}

Osteoclasts are essential for bone remodelling and are normally found at the bone surface, where they create resorption pits. Joints are formed where the extremities of bones meet and articulate. Cartilage is composed of chondrocytes and a collagenous extracellular matrix rich in proteoglycan and collagen that lines and lubricates the joint. The bone-cartilage interface comprises the deep layer of non-calcified cartilage, the calcified cartilage, and the underlying subchondral bone. Osteoarthritis (OA) is characterized by a degeneration of articular cartilage and the crosstalk between cartilage and subchondral bone plays an important role in the development and progression of OA. Direct biochemical crosstalk or cellular contact between cartilage and bone might modify the cellular environment and influence disease progression. Whether osteoclasts degrade cartilage has been little explored and is a challenging question. We aim to characterize how osteoclasts can affect cartilage degradation.

Tissue sections from osteoarthritis and giant cell tumor of bone (GCTB) showed osteoclasts in contact with cartilage. Cartilage pieces from OA patients and dentine (positive control) were used as a substrate for osteoclasts. We demonstrated the capacity of monocyte-derived osteoclasts and primary osteoclasts (giant 
cells) from GCTB to release glycosaminoglycan and collagen from cartilage. To understand how osteoclasts degrade the cartilage extracellular matrix, we used a RANKL-inhibitor (OPG), an acidification inhibitor (Bafilomycin), an MMP inhibitor (GM6001), and a Cathepsin K inhibitor (E64). Inhibition of osteoclast resorption activity on dentine validated the efficacy of the inhibitors. Only GM6001 reduced cartilage degradation $(55 \% \pm 3.5 \%, p<0.01)$, highlighting that osteoclasts use MMPs to degrade cartilage by release of proteoglycans. To find a potential candidate involved in cartilage degradation, osteoclasts were cultured on either plastic, dentine, or cartilage. We then looked at the expression of 15 genes including genes known to be expressed in active osteoclasts and different MMPs. MMP-1, 3, 10, and 13 were highly expressed in osteoclasts cultured on cartilage compared with either plastic or dentine.

Understanding and controlling the activity of osteoclasts would present an attractive target for further research and might represent an interesting perspective for diseases characterized by cartilage degeneration.

\section{P9}

Impact of mild and moderate/severe vertebral fractures on physical activity: a 5-year prospective study based on a cohort of older women in the UK

\section{Usama A Al-sari, Jon H Tobias, and Emma M Clark}

Bristol Medical School, University of Bristol, Bristol, UK

Objective: Little is known about the long-term impact of vertebral fractures (VFs) on physical activities. There is also uncertainty over the clinical significance of mild VF. Therefore, the aim of the present study was to evaluate the prospective association between baseline VF and future physical activity.

Methods: In a 5-year prospective study from a mixed community and secondary care in the UK, 700 women aged $>50$ years (mean age 68.2 years) with baseline spine images provided data on self-reported physical activities after 5.4 years follow-up. VFs were identified at baseline on radiographs or DXA-based vertebral fracture assessment (VFA) by a quantitative morphometric approach and defined as a moderate/severe VF ( $\geq 25 \%$ height decrease) or mild VF (20\% to $24.9 \%$ height decrease). Physical activity data were collected at follow-up by self-completion questionnaires. Multivariate logistic regression was used to determine the association between presence of VF and various physical activities while adjusting for potential confounders.

Results: 286 women without, 58 with mild, and 69 with moderate/severe VF were recruited to this study. Those with mild and moderate/severe fracture were older than women without fracture (mean age of 69.5 and 72.2 years versus 66.8 years) and had more concomitant diseases at baseline. At 5.4 years' follow-up, women with moderate/severe fracture reported shorter walking duration compared with those without fracture, even after adjusting for potential confounders (OR $=2.96,95 \% \mathrm{Cl} 1.11-7.88, p=0.030$ ). No association was found between presence of mild VFs and reduced physical functioning at follow-up.

Conclusion: This is the first study of older women from the UK that explored the prospective association between VF and physical activity duration. Both moderate/severe and mild VF are inversely related to subsequent activity.

\section{P10}

Limb bones scale similarly despite forelimb-hindlimb load asymmetry in bipedal hopping

Michael Doube, ${ }^{1,2}$ Alessandro A Felder, ${ }^{2}$ Melissa Y Chua, ${ }^{1}$ Kalyani Lodhia, ${ }^{2}$ Michał M Kłosowski, ${ }^{1}$ John R Hutchinson, ${ }^{3}$ and Sandra J Shefelbine ${ }^{1,4}$

${ }^{1}$ Department of Bioengineering, Imperial College London, London, UK

${ }^{2}$ Skeletal Biology Group, Comparative Biomedical Sciences, The Royal Veterinary College, London, UK

${ }^{3}$ Structure and Motion Laboratory, Comparative Biomedical Sciences, The Royal Veterinary College, London, UK

${ }^{4}$ Department of Mechanical \& Industrial Engineering, Northeastern University, Boston, MA, USA

Bone adaptation is modulated by the timing, direction, rate, and magnitude of mechanical loads, and displays a saturation effect whereby no further bone formation response occurs after a few tens of load cycles. To investigate whether frequent slow, or infrequent fast, gaits could dominate bone adaptation to load, we compared scaling of the limb bones from two mammalian herbivore clades that use radically different high-speed gaits, bipedal hopping and quadrupedal galloping. Forelimb and hindlimb bones were collected from 20 artiodactyl (sheep, deer, and kin) and 15 macropodiform (kangaroos and kin) species (body mass $M$ 1.05-1536 kg) and scanned in clinical computed tomography or X-ray microtomography. The cross-sectional parameter second moment of area $\left(I_{\max }\right)$ was measured on every transverse image slice using BoneJ's Slice Geometry, and bone length $(I)$ was measured using Pythagoras' theorem. Scaling relations $\left(y=a x^{b}\right)$ were calculated for I versus $M$ for each bone, and for $I_{\max }$ versus $M$ and $I_{\max }$ versus $I$ for every $5 \%$ of length of each bone. $I_{\max }$ versus $M$ scaling relationships were broadly similar between clades despite the macropodiform forelimb being nearly unloaded, and the hindlimb highly loaded, during bipedal hopping. I max versus / and / versus $M$ scaling were related to locomotor and behavioral specializations such as grasping with the manus in Macropodiformes. Low strains and strain rates engendered during common slow gaits may be sufficient to maintain bone mass across a wide range of species. Occasional high-intensity gaits might not break through the load sensitivity saturation engendered by frequent low-intensity gaits. This work was supported by BBSRC grants to SJS (BB/F001169/1) and JRH (BB/F000863/1). AF was supported by an RVC PhD studentship.

\section{P11}

A tale of two phosphatases: dissecting the roles of PHOSPHO1 and TNAP during skeletal biomineralization

\section{Scott Dillon, ${ }^{1}$ Fabio Nudelman, ${ }^{2}$ and Colin Farquharson ${ }^{1}$ \\ ${ }^{1}$ The Roslin Institute, University of Edinburgh, Edinburgh, UK \\ ${ }^{2}$ School of Chemistry, University of Edinburgh, Edinburgh, UK}

Skeletal biomineralization in vertebrates is a complex, highly regulated process that generates the intricate hierarchical and composite structure of bone tissue. At the nanostructural level, the collagen fibril framework allows templating and nucleation of the calcium phosphate mineral phase. Generation of 
inorganic phosphate $(\mathrm{Pi})$ for mineral formation has historically been attributed to tissue non-specific alkaline phosphatase (TNAP); however, recent research has demonstrated the critical role of PHOSPHO1. This work aims to elucidate how these two phosphatases function in concert to regulate mineralization in the skeleton during development.

Embryonic (E) 14- to 17-day-old wild-type C67BL/6J embryos were studied. Skeletal morphogenesis was examined using whole-mount Alizarin Red staining and optical projection tomography (OPT). Histological investigation allowed microscopic characterization of tissue architecture. Immunohistochemistry for TNAP and PHOSPHO1 was undertaken for protein localization. Calvaria and lower limbs were dissected and RNA isolated for Phospho1 and Akp2 gene expression analysis by RT-qPCR. Samples were embedded in resin and ultrastructural characterization carried out using transmission electron microscopy.

Skeletal ossification in both calvaria and lower limbs was observed to begin at E15, with rapid bone formation between E15-E16. This was confirmed microscopically with increased osteoid and collagen deposition, co-localizing with the presence of phosphate, and an increased number of osteoblastic cells. Analysis of gene expression showed differential regulation of expression between the sites. Upregulation of both Phospho1 $(\mathrm{p}=0.094)$ and $A k p 2(p=0.053)$ occurred in the lower limb over the developmental time course; however, these increases were not statistically significant. Expression of both genes was significantly higher in calvaria compared with the lower limb $(p<0.001)$ with no association between developmental age and site, indicating similar patterns of expression over time. IHC staining for both phosphatases showed divergent organization of the proteins within the tissue architecture consistent at both anatomical sites, with PHOSPHO1 localizing to the extracellular matrix adjacent to osteoblastic cells, whereas TNAP localized to bony spicules in the later time points. Together these data may indicate differential regulation of phosphatase expression, and therefore $\mathrm{Pi}$ generation, between intramembranous and endochondral modes of ossification.

\section{P12}

Human decellularized blood vessel matrices promote bone repair in an ex vivo bone defect model

\section{Janos Kanczler, ${ }^{1}$ Stefanie Inglis, ${ }^{1}$ Karl Schneider, ${ }^{2}$ Heinz Redl, $^{3}$ and Richard Oreffo ${ }^{1}$}

\author{
${ }^{1}$ Bone and Joint Research Group, University of Southampton, \\ Southampton, UK \\ ${ }^{2}$ Department of Biomedical Research, Medical University of Vienna, \\ Vienna, Austria \\ ${ }^{3}$ Experimental and Clinical Traumatology/AUVA Research Centre, \\ Ludwig Boltzmann Institute, Vienna, Austria
}

Introduction: Decellularized tissue matrices offer a valuable substitute for biomimetic scaffolds in tissue engineering given their innate preservation of native extracellular matrix (ECM) and integral tissue architecture. We have examined decellularized blood vessel grafts from human placenta chorions (placental vessel sleeves [PVS]) as a periosteal protective sleeve to enhance the repair of a bone defect.

Methods: 1-mm precision drill defects were created middiaphysis in E18 chick femurs and implanted with or without an endothelial cell pellet construct (HUVEC). Human biocompatibility-tested decellularized placenta sleeves were cut to size and placed over the femur defects. PVS femurs were maintained in organotypic culture for a period of 10 days. Micro-CT scanning was performed pre- and post-culture and samples fixed and processed for histological analysis.

Results: Imaging at day 10 revealed a visible reduction in the defect area in femurs covered with the PVS. Significant increases in bone volume (BV) in sleeved treatment groups $(0.52 \pm 0.2$ $\mathrm{mm}^{3}$ ) were found compared with non-sleeved femur groups $\left(-0.10 \pm 0.2 \mathrm{~mm}^{3} ; p=0.0001 ; n=15\right)$. Histological analysis demonstrated extensive collagen expression with widespread integration of the decellularized sleeves with the bone collar with enhanced conservation of bone architecture within the PVS sleeves. Dense bands of proteoglycans were observed between the sleeves and the periosteal bone and cells within these regions expressed high levels of collagen II as well as the hypoxia marker, hypoxia-inducible factor 1-alpha (HIF-1 $\alpha$ ).

Conclusion: Decellularized vessel grafts (PVS) applied to bone defects analyzed within a 3D organotypic model demonstrated extensive integration with the bone and significant bone repair at the site of the skeletal defect. PVS femurs demonstrated higher levels of bone formation regardless of the presence of pellet constructs, indicating the potential of decellularized grafts alone to augment bone healing. Furthermore, in combination with cell pellets, the migratory activity of HIF- $1 \alpha$-positive cells was observed. In summary, decellularized vascular matrices offer a promising therapeutic strategy in surgical tissue replacement promoting biocompatibility and, critically, an ECM-rich niche environment to augment bone repair.

Disclosures: None declared.

\section{P13}

Slc38a10 is a novel genetic determinant of osteoblast proliferation and bone mineral density

\section{Andrea S Pollard, ${ }^{1}$ Apostolos Gogakos, ${ }^{1}$ John G Logan, ${ }^{1}$ Davide Komla Ebri, ${ }^{1}$ Penny C Sparkes, ${ }^{1}$ Natalie C Butterfield, ${ }^{1}$ Victoria D Leitch, ${ }^{1}$ Sanger Mouse Pipelines, ${ }^{2}$ Peter I Croucher, ${ }^{3}$ JH Duncan Bassett, ${ }^{1}$ and Graham R Williams $^{1}$}

\author{
${ }^{1}$ Molecular Endocrinology Laboratory, Imperial College London, \\ London, UK \\ ${ }^{2}$ Wellcome Trust Sanger Institute, Hinxton, UK \\ ${ }^{3}$ Bone Biology Division, Garvan Institute of Medical Research, \\ Sydney, Australia
}

Low bone mineral density (BMD) is a key risk factor for osteoporosis and fracture, resulting in major societal and financial burdens to the NHS. Although BMD is highly heritable, over $85 \%$ of the phenotype variance remains unexplained. We hypothesized that high-throughput skeletal phenotyping of knockout mice generated by the International Knockout Mouse Consortium would identify new genetic determinants of bone structure and strength. Sixteen-week-old female sodiumcoupled neutral amino acid transporter $10\left(\right.$ S/c38a $\left.10^{-/-}\right)$knockout mice were identified to have decreased body weight, bone length, and BMD by this screen.

Comprehensive phenotyping of juvenile and adult S/c38a10-mice ( $n=5$ per sex, per genotype) using X-ray microradiography and micro-computed tomography demonstrated short stature 
throughout postnatal growth and in adults ( $p<0.05$, ANOVA) with decreased bone mineral content ( $p<0.001$, KolmogorovSmirnov test) and reduced cortical ( $p<0.001$, ANOVA) and trabecular bone thickness ( $p<0.001$, ANOVA). Despite the clear deficit in skeletal growth, histology of $S / c 38 a 10^{-/-}$epiphyseal growth plates was normal. Histomorphometry revealed no difference in osteoclast numbers, but $S / \mathrm{c}_{38} \mathrm{a} 10^{-/}$mice had profound decreases in mineral apposition (40\%, $p<0.05, t$ test) and bone formation $(60 \%, p<0.05, t$ test) rates compared with wild-type mice. S/c38a10 $/-$ primary osteoblasts had decreased proliferation ( $p<0.05, t$ test) compared with wild-type but produced extracellular matrix and mineralized normally once confluent.

Taken together, these data suggest that impaired skeletal growth and low BMD in S/C38a10 $0^{-/-}$mice result primarily from an osteoblast defect. Slc38a10 is a sodium-coupled glutamine and aspartate transporter that has been implicated in amino acid sensing and transport and the regulation of mTORC1. Thus, SIc38a10 may contribute to a mechanism by which skeletal growth and mineralization are regulated in response to nutrient availability. Identification of the molecular mechanisms underlying this process may identify new drug targets involved in the maintenance of BMD and bone strength.

\section{P14}

In vivo skeletal regeneration using a nanocomposite silicate-based bioink

\section{Gianluca Cidonio, ${ }^{1,2}$ Tilman Ahlfeld, ${ }^{3}$ Michael Glinka, ${ }^{1}$ Yang- Hee Kim, ${ }^{1}$ Stuart Lanham, ${ }^{1}$ Janos Kanczler, ${ }^{1}$ Shoufeng Yang, ${ }^{2}$ Jonathan Dawson, ${ }^{1}$ Michael Gelinsky, ${ }^{3}$ and Richard Oreffo $^{1}$}

\begin{abstract}
${ }^{1}$ Bone and Joint Research Group, Centre for Human Development, Stem Cells and Regeneration, Institute of Developmental Sciences, University of Southampton, Southampton, UK

${ }^{2}$ Engineering Materials Research Group, Faculty of Engineering and the Environment, University of Southampton, Southampton, UK ${ }^{3}$ Centre for Translational Bone, Joint and Soft Tissue Research, University Hospital Carl Gustav Carus and Faculty of Medicine, Technische Universität Dresden, Dresden, Germany
\end{abstract}

Introduction: An ideal hydrogel for skeletal biofabrication should aim to preserve cell viability during printing and sustain proliferation right after. Here we have developed a nanocomposite bioink to allow high printing fidelity of human bone marrow stromal cells (HBMSCs) with elevated cell viability in vitro and functionality in vivo.

Objectives: (i) To fabricate and characterize HBMSCs-laden constructs in vitro; (ii) to evaluate integration and vascular infiltration in an ex vivo model; (iii) to implant and investigate ectopic bone formation of BMP-2 loaded 3D clay-based scaffolds.

Methods: $5 \times 10^{6} \mathrm{HBMSCs} \mathrm{ml}^{-1}$ were encapsulated in nanoclay (Laponite), alginate, and methylcellulose bioink, printed, and evaluated for viability after live/dead confocal analysis. 3Dprinted scaffolds were implanted in a vascularized chorioallantoic membrane (CAM) of a developing chick egg and vascular infiltration assessed by Chalkley score and histology. MF-1 mice were anesthetized, three constructs were implanted on each side, and computed tomography (CT) was carried out using Bruker Skyscan 1176 after 28 days.
Results: Cell viability was investigated at days $1(81.1 \% \pm 13.3)$ $7(88.0 \% \pm 6.7), 14(88.3 \% \pm 9.2)$, and 21 (88.8 $\% \pm 8.0)$. Confocal analysis demonstrated HBMSCs proliferation over time. Alkaline phosphatase staining at day 1 confirmed HBMSCs differentiation. Investigation of clay-based 3D-printed scaffolds in an ex vivo CAM model showed integration and vasculature infiltration after 7 days. BMP-2-loaded and -free biofabricated scaffolds implanted within an in vivo subcutaneous mouse model showed significantly higher mineralized tissue volume when compared with bulk alginate BMP-2-loaded and -free hydrogel $\left(15.17 \pm 1.98 \mathrm{~mm}^{3}[p<0.0001]\right.$ and $13.75 \pm 3.98 \mathrm{~mm}^{3}[p<$ 0.0001], respectively). Micro-CT analysis of BMP-2-loaded 3Dprinted clay scaffolds demonstrated a significant fivefold increase in bone formation and bone mineral density compared with alginate-BMP-2 control ( $p<0.0001)$.

Conclusion: We have successfully demonstrated viability and functionality of biofabricated clay-based scaffolds. Implantation in CAM and subcutaneous models showed chick vessels infiltration and new mineralized tissue formation, respectively, proving clinical relevance of this new nanoclay biofabrication 3D platform with implications for orthopedic reparative strategies.

\section{P15}

Bone induction in a murine subcutaneous model using nanoclay gel and bone morphogenic protein: an optimization study

\section{Josephine McEwan, Janos Kanczler, Stuart Lanham, Julia Wells, Jonathan Dawson, and Richard Oreffo}

Bone and Joint Research Group, Centre for Human Development, University of Southampton, Southampton, UK

Introduction: For almost three decades, bone morphogenic protein-2 (BMP-2) has been demonstrated to be a key regulatory molecule able to induce bone formation. BMP-2 is licensed for use, clinically, in spinal arthrodesis and open tibial fractures. However, dose-dependent side effects, including osteolysis, swelling, and unwanted heterotopic ossification have hindered BMP-2 usage in recent practice. Clay nanoparticle gels (Laponite) have been shown to localize BMP-2 and induce bone at physiological doses. In the current study, we investigated the optimum concentrations and dosage of Laponite and BMP-2 required to induce ectopic bone using a murine subcutaneous implant model.

Methods: Laponite combined with BMP-2 on an absorbable collagen sponge (ACS) was implanted in a murine subcutaneous model. The Laponite concentrations of $0 \%, 1.4 \%, 2.1 \%$, and $2.8 \%$ and BMP-2 doses $0,0.05,0.5$, and $5 \mu \mathrm{g} / \mathrm{mL}(n=6$ for each treatment combination) were examined. Micro-CT scanning was performed at 2, 4, 6, and 8 weeks to quantify the volume of induced ectopic bone.

Results: The highest concentration of Laponite (2.8\%) was observed to induce significantly higher volumes of ectopic bone compared with $0 \%$ Laponite $(p<0.005), 1.4 \%(p<0.005)$, and $2.1 \%(p<0.01)$ at $5 \mu \mathrm{g} / \mathrm{mL}$ dose of BMP-2. The mean volumes of induced bone were $0,0.131,0.249$, and $1.141 \mathrm{~mm}^{3}$ for $0 \%, 1.4 \%$, $2.1 \%$, and $2.8 \%$ Laponite, respectively. Two-way ANOVA test demonstrated at week 4 that Laponite concentration and BMP-2 dose significantly affected the volume of ectopic bone formed ( $p$ $<0.01$ and $p<0.01$, respectively). The effect of Laponite concentration on bone induction remained significant at 
8 weeks $(p<0.02)$, whereas the effect of BMP-2 dose was not significant at this extended time point.

Conclusion: The current study demonstrates that Laponite augments the osteoinductive action of BMP-2. Significantly enhanced bone formation was observed at the highest nanoclay concentration examined, with Laponite exerting an osteoinductive factor across the study time frame compared with BMP2. This study has significant implications for the application of nanoclays for skeletal growth factor delivery, such as BMP-2, at physiological concentrations, and opportunities for improved safe, efficacious, and cost-effective strategies for bone augmentation in orthopedics.

\section{P16}

Osteoporosis as a risk factor for the occurrence of frailty: a 4year follow-up of the ROAD study

\author{
Noriko Yoshimura, ${ }^{1}$ Shigeyuki Muraki, ${ }^{1}$ Hiroyuki Oka, ${ }^{2}$ \\ Toshiko lidaka, ${ }^{1}$ Rie Kodama, ${ }^{3}$ Chiaki Horii, ${ }^{3}$ Hiroshi \\ Kawaguchi, ${ }^{4}$ Kozo Nakamura, ${ }^{5}$ Toru Akune, ${ }^{5}$ and Sakae \\ Tanaka $^{3}$
}

${ }^{1}$ Department of Prevention Medicine for Locomotive Organ Disorders, The University of Tokyo, Tokyo, Japan

${ }^{2}$ Department of Medical Research and Management for Musculoskeletal Pain, The University of Tokyo, Tokyo, Japan

${ }^{3}$ Department of Orthopaedic Surgery, Sensory and Motor System Medicine, Gradu, The University of Tokyo, Tokyo, Japan

${ }^{4}$ JCHO Tokyo Shinjuku Medical Center, Tokyo, Japan

${ }^{5}$ National Rehabilitation Center for Persons with Disabilities, Saitama, Japan

Objective: To determine whether osteoporosis contributes significantly to the subsequent occurrence of frailty, the cumulative incidence of frailty was estimated and assessed.

Methods: The second survey of the Research on Osteoarthritis/ Osteoporosis Against Disability (ROAD) study-a large-scale population-based cohort study-was conducted between 2008 and 2010. Among 1099 participants of the second survey for the ROAD study, 1083 subjects (aged $\geq 60$ years, 372 men, 711 women) completed all the examinations for frailty and osteoporosis. The third survey was conducted 4 years after the second survey. Among the 1083 participants in the second survey, aged 60 years and older when frailty was assessed, 749 (69.2\%, 248 men, 501 women) subjects completed all the frailty and osteoporosis examinations. Among the 749 who completed, 20 (4 men, 16 women) had already been diagnosed with frailty in the second survey. Therefore, the final number of subjects at risk for the occurrence of frailty was 729 (244 men, 485 women). Frailty was defined using Fried's definition with the following five variables: unintentional weight loss, self-reported exhaustion, low physical activity, weakness (grip strength $<26 \mathrm{~kg}$ in men, $<18 \mathrm{~kg}$ in women), and slowness (slow walking speed, the usual gait speed $\leq 0.8 \mathrm{~m} / \mathrm{s}$ ). Those who reported positively on three or more of the five factors were judged to be frail. Osteoporosis was defined based on the World Health Organization criteria.

Results: The cumulative annual incidence of frailty was $1.2 \% / \mathrm{yr}$ (men, 0.8\%/yr; women, 1.3\%/yr). After adjustment for confounding factors, a logistic regression analysis using the occurrence of frailty as the objective variable and the presence of osteoporosis as the explanatory variable indicated that the presence of osteoporosis was significantly associated with the occurrence of frailty in the near future (odds ratio $=3.24 ; 95 \%$ confidence interval 1.38-7.63; $p<0.01$ ).

Conclusion: This prospective study suggests that prevention of osteoporosis may be useful in reducing the risk of frailty, as well as osteoporotic fractures in the future.

\section{P17}

TGF $\beta$ inhibition in combination with chemotherapy repairs existing lytic bone lesions in a novel plateau phase model of multiple myeloma

\author{
Alanna Green, ${ }^{1}$ Katie Hudson, ${ }^{1}$ Jenny Down, ${ }^{1}$ Darren Lath, ${ }^{1}$ \\ Holly Evans, ${ }^{1}$ Julia Paton-Hough, ${ }^{1}$ Simon Tazzyman, ${ }^{1}$ Matt \\ Fisher, ${ }^{1}$ John Snowden, ${ }^{2}$ Andrew Chantry, ${ }^{1}$ and Michelle \\ Lawson $^{1}$ \\ ${ }^{1}$ Oncology and Metabolism, The University of Sheffield, Sheffield, \\ UK \\ ${ }^{2}$ Haematology, Sheffield Teaching Hospitals NHS Foundation Trust, \\ Royal Hallamshire Hospital, Sheffield, UK
}

Multiple myeloma (MM) causes a destructive bone disease in $>85 \%$ of patients and current therapies do little to repair existing bone damage. We previously identified that combined bone anabolic and anti-resorptive therapy repairs osteolytic lesions in mice with high tumor load. In patients, if bone repair agents were given, they would be administered in combination with chemotherapy. Thus, this study aimed to determine if bone recovers after chemotherapy and if this is enhanced by bone anabolic therapy.

Human U266-GFP-luc MM cells were i.v. injected into NSG mice. After tumor and bone lesion development, mice were administered first-line chemotherapeutics (bortezomib \pm lenalidomide) \pm a bone anabolic (SD208; transforming growth factor $\beta$ receptor 1 inhibitor) or vehicles for 2 weeks. Tumor and bone lesions were monitored in vivo by bioluminescence imaging $(B L I)$, serum paraprotein ELISAs, and $\mu C T$. Flow cytometry, histomorphometry, $\mu C T$, TRAP, and P1NP ELISAs and QPCR were performed for endpoint analyses.

Chemotherapy significantly reduced total body tumor burden and paraprotein and increased survival. Combined chemotherapy was more effective than either given alone, reducing tumor to levels undetectable by BLI and paraprotein. However, flow cytometry revealed low tumor levels of $100 \mathrm{MM}$ cells $/ 10^{6}$ bone marrow cells. Bone lesions developed $\sim 8$ weeks after tumor inoculation. Vehicle-treated mice exhibited progressive bone lesion development and virtually no trabecular bone at endpoint. Lesions in mice administered bortezomib \pm lenalidomide were unchanged after 1 week but began to repair after 2 weeks, with significantly reduced TRAP + osteoclasts and increased osteoblasts, indicating recovery of bone. Mice treated with chemotherapy + anabolic SD-208 exhibited enhanced repair of bone lesions, with partial repair of perforating cortical lesions on all tibial surfaces within 1 week and complete repair of lesions within 2 weeks. SD-208 also significantly increased trabecular bone volume after 2 weeks.

This study identified SD-208 enhances MM bone lesion repair when combined with first-line chemotherapeutics. Future studies combining SD-208 and chemotherapy with antiresorptive therapy will identify optimum treatment regimens for translation of bone anabolic therapy into MM clinical trials. 


\section{P18}

Photoperiod-induced central actions of thyroid hormone are essential for medullary bone formation in Japanese quail

Natalie C Butterfield, ${ }^{1}$ Justyna Miszkiewicz, ${ }^{1}$ Anne-Tounsia Adoum, ${ }^{1}$ John Logan, ${ }^{1}$ Victoria Leitch, ${ }^{1}$ Takashi Yoshimura, ${ }^{2}$ Duncan Bassett, ${ }^{1}$ and Graham Williams ${ }^{1}$

${ }^{1}$ Molecular Endocrinology Laboratory, Imperial College, London, UK ${ }^{2}$ Laboratory of Animal Physiology, Graduate School of Bioagricultural Sciences, Nagoya University, Nagoya, Japan

Seasonal reproduction enables animals outside tropical regions to rear offspring in a favorable environment. Increasing day length triggers a hypothalamic relay involving thyrotropin, the type 2 deiodinase enzyme and thyroid hormone, which activates the hypothalamic-pituitary-gonadal axis to induce reproductive competence. Photoperiod regulates calcium metabolism and the egg-laying cycle in the Japanese quail (Coturnix japonica), and we hypothesized that activity of this relay would have major consequences for bone mineralization and strength. Quails were housed in long (20-hour light, 4-hour dark) or short (6-hour light, 18-hour dark) day conditions for up to 12 weeks and skeletal consequences were determined by X-ray microradiography, micro-CT, electron microscopy, histomorphometry, and biomechanical testing ( $n=10$ per sex, per group). Both ovary and testis weights increased $>10$-fold ( $p<0.001$, ANOVA) after longday compared with short-day exposure. Long-day exposure in females resulted in massive increases in bone mineral content and mineralization ( $p<0.001$, Kolmogorov-Smirnov test) and bone strength and stiffness ( $p<0.001$, ANOVA), as a consequence of medullary bone formation. By contrast, medullary bone was absent in females exposed to short-day length and never observed in males. Medullary bone was a highly vascular and dynamic tissue, characterized by osteoclast resorption pits and mineral apposition fronts covering almost the entire bone surface. Reversal of photoperiod resulted in (i) rapid ovarian regression and loss of medullary bone in females previously exposed to long-day conditions, and (ii) rapidly increased ovarian size and induction of medullary bone formation in females previously exposed to short days. These data demonstrate that the skeleton is exquisitely sensitive to photoperiod during avian seasonal reproduction, and the central actions of thyroid hormone are essential for medullary bone formation. Elucidation of mechanisms that mobilize calcium and synchronize egg shell formation during the daily reproductive cycle may identify novel pathways that couple bone resorption and formation.

\section{P19}

Gpc6: a novel determinant of bone mineral density in osteoporosis

Naila S Mannan, ${ }^{1}$ Victoria D Leitch, ${ }^{1}$ John G Logan, ${ }^{1}$ AnneTounsia Adoum, ${ }^{1}$ Hannah F Dewhurst, ${ }^{1}$ Andrea S Pollard, ${ }^{1}$ Penny C Sparkes, ${ }^{1}$ Elena J Ghirardello, ${ }^{1}$ Rebecca Allen, ${ }^{1}$ Natalie C Butterfield, ${ }^{1}$ Sangar Mouse Pipelines, ${ }^{2}$ David Komla-Ebri, ${ }^{1}$ Katharine F Curry, ${ }^{1}$ Peter I Croucher, ${ }^{3}$ Graham R Williams, ${ }^{1}$ JH and Duncan Bassett ${ }^{1}$

${ }^{1}$ Molecular Endocrinology Laboratory, Imperial College London, London, UK
${ }^{2}$ Sanger Institute, Wellcome Trust, Cambridge, UK

${ }^{3}$ Bone Biology Division, Garvan Institute of Medical Research, Sydney, Australia

The Origins of Bone and Cartilage Disease project is funded by a Wellcome Trust Strategic Award to undertake rapid-throughput skeletal phenotyping of knockout mice generated at the Sanger Institute, as part of the International Knockout Mouse Consortium. Nineteen parameters of bone structure and strength are analyzed by X-ray microradiography, micro-computed tomography, and biomechanical testing in 16-week-old females from individual knockout mouse lines and compared with reference data obtained from $>300$ wild-type mice of identical genetic background. This approach tested the hypothesis that rapidthroughput screening of knockout mice will facilitate discovery of novel genes implicated in the pathogenesis of osteoporosis. We identified that Gpc6-/- knockout mice lacking glypican-6, a glycosylphosphatidylinositol-anchored membrane-bound heparan sulfate proteoglycan, had decreased lengths of the femur $(-1.95 \mathrm{SD}$, permuted $p=0.06)$ and vertebrae $(-2.17 \mathrm{SD}$ permuted $p=0.016)$; together with increased bone mineral content $\left(+2.4 \mathrm{SD}\right.$, permuted $\left.p=3 \times 10^{-4}\right)$ and cortical bone thickness $\left(+2.3 \mathrm{SD}\right.$, permuted $\left.p=5 \times 10^{-3}\right)$. These structural abnormalities resulted in increased strength and elasticity of long bones (yield load $+2.1 \mathrm{SD}$, permuted $p=8 \times 10^{-3}$ ). A genomewide association study (GWAS) in 142,487 individuals from the UK Biobank also identified the GPC6 locus to be strongly associated with bone mineral density estimated by quantitative ultrasound of the calcaneus.

Loss-of-function mutations in GPC6 in humans cause omodysplasia-1 (OMIM 258315), a rare autosomal recessive skeletal dysplasia that causes micromelia and craniofacial abnormalities consistent with GPC6 expression in chondrocytes. Limited information is available regarding adult manifestations of omodysplasia-1 and a role for GPC6 in the control of bone mass and strength has not been recognized previously. Recent studies indicate a direct role for GPC6 in the regulation of Wnt signaling and thus suggest one pathway through which GPC6 is involved in the pathogenesis of osteoporosis. Elucidation of the molecular mechanisms that cause increased bone mass and strength in Gpc6-/- mice may identify novel therapeutic approaches for prevention and treatment of osteoporosis.

\section{P20}

Age-related changes in native human bone marrow mesenchymal stem cells

\section{Payal Ganguly, Jehan J El-Jawhari, Agata N Burska, Frederique Ponchel, Peter V Giannoudis, and Elena A Jones}

Leeds Institute of Rheumatic and Musculoskeletal Medicine, St James's University Hospital, University of Leeds, Leeds, UK

Objectives: Aging of bone is associated with increased risk of fracture in the elderly and increased prevalence of osteoporosis and osteoarthritis. With aging, the process of bone formation and remodeling is affected. Mesenchymal stem cells (MSCs) are precursors for osteocytes and chondrocytes. The objective of this study was to investigate the changes in native human bone marrow (BM) MSCs with aging.

Methods: BM aspirate collected from donors (19 to 89 years old) was directly plated onto $60-\mathrm{mm}$ dishes in duplicate for 
enumeration of MSCs by colony-forming unit-fibroblast (CFU-F) assay or processed for cytometric enumeration of MSCs by flow cytometry using the $\mathrm{CD} 45^{\text {low }} \mathrm{CD} 271^{\text {high }}$ phenotype $(n=55)$. Samples with at least 30 colonies/dish were used for colony area and density analysis using ImageJ ( $n=5$ each from younger [20 to 40] and older [above 60] donors). Fluorescence-activated cell sorting was used to study gene and surface marker expression in CD45 ${ }^{\text {low }}$ CD271 $1^{\text {high }}$ MSCs.

Results: There was a fourfold decline in CFU-F numbers in older $(p<0.0001)$ and intermediate-age ( 41 to $59 ; p<0.0001)$ donors compared with younger donors. A similar decline in the intermediate group $(p<0.05)$ compared with the younger group was detected by flow cytometry. Frequency distribution of colony area revealed no significant differences with over $60 \%$ colonies being between 5 and $15 \mathrm{~mm}^{2}$. Interestingly, the density of these most frequent colonies was $50 \%$ lower in older donors compared with younger donors $(p<0.0001)$. Distinct subsets $\left(\right.$ CD106 ${ }^{\text {low }} \mathrm{CD} 295^{\text {high }}$ and CD146 $\left.{ }^{\text {low }} \mathrm{C} \times 43^{\text {high }}\right)$ were present within $\mathrm{CD} 45^{\text {low }} \mathrm{CD} 271^{\text {high }}$ MSC population and an 18-fold decline in $\mathrm{CD} 146^{\text {low }} \mathrm{C} \times 43^{\text {high }}$ subpopulation was observed in older donors compared with younger donors ( $n=7$ and 9, respectively, $p=0.0041$ ). Gene expression of fat-associated genes (FABP4, PPAR-g, Leptin receptor) and osteoprotegerin displayed a trend to increase in older donors, whereas RANKL expression was downregulated ( $n=5$ in each group).

Conclusion: These data indicate a gradual decline of BM-MSC numbers notable from the fifth decade of life. In all age groups, CFU-F colonies remained heterogeneous in size, but same-sized colonies in older donors were less dense, suggesting reduced MSC proliferative capacity at the single-cell level. Gene expression differences indicated the increased bias toward adipogenesis and alterations in bone remodeling regulation in aged BM MSCs.

\section{P21}

Fam73b is essential for skeletal growth and the maintenance of bone mass and strength

Davide Komla-Ebri, ${ }^{1}$ Apostolos Gogakos, ${ }^{1}$ Penny Sparkes, ${ }^{1}$ John G Logan, ${ }^{1}$ Sanger Institute Mouse Pipelines, ${ }^{2}$ Peter I Croucher, ${ }^{3}$ JH Duncan Bassett, ${ }^{1}$ and Graham R Williams ${ }^{1}$

\author{
${ }^{1}$ Molecular Endocrinology Laboratory, Imperial College London, \\ London, UK \\ ${ }^{2}$ Wellcome Trust Sanger Institute, Cambridge, UK \\ ${ }^{3}$ Bone Biology Division, Garvan Institute of Medical Research, \\ Sydney, Australia
}

Fam73b encodes mitoguardin 2 (Miga2), a protein that regulates mitochondrial fusion, morphology, and function. We performed rapid-throughput skeletal phenotype analysis of knockout mice generated by the International Knockout Mouse Consortium at the Wellcome Trust Sanger Institute and identified Fam73b as a novel genetic determinant of bone mass and strength. Sixteenweek-old female Fam $73 b^{-1-}$ homozygous knockout mice had weak and flexible bone with low mineral content, features that characterize the skeletal abnormalities in osteoporosis.

To determine the role of mitoguardin 2 in skeletal development and bone maintenance, we performed detailed phenotyping of Fam $73 b^{-1-}$ mice. X-ray microradiography and micro-computed tomography demonstrated short stature throughout growth and in adulthood, decreased bone mineral content at post-natal days P14 ( $-11.17 \%, p<0.001, n=15), \mathrm{P} 28(-8.66 \%, p<0.001$, $n=14$ ), P70 (females: $-3.05 \%, p<0.001, n=11$; males: $-3.21 \%$, $p<0.01, n=15$ ), and P183 (females: $-4.15 \%, p<0.001, n=10$; males: $-3.43 \%, p<0.01, n=12$ ), and decreased cortical bone thickness at P70 (females: $-5.91 \%, p<0.05, n=11$; males: $-6.03 \%, p<0.05, n=15$ ) and P183 (females: $-6.56 \%, p<0.01$, $n=10$; males: $-4.22 \%, p<0.05, n=12$ ) in ${\text { Fam } 73 b^{-/}}_{\text {mice }}$ compared with wild type. Three-point bend biomechanical

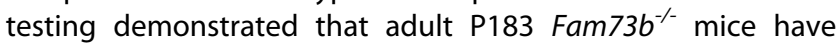
markedly decreased bone strength and stiffness. Histomorphometric analysis of the tibial growth plate at P14 and tartrateresistant acid phosphatase staining of tibial sections at P70 demonstrated no differences between $\mathrm{Fam}_{3} \mathrm{~b}^{-/-}$and wild-type mice, suggesting the abnormal phenotype in $\mathrm{Fam}_{73 \mathrm{~b}^{-/}}$mice may be due to defective osteoblasts rather than abnormalities of chondrocyte or osteoclast function. Primary culture studies are underway to investigate further and preliminary data indicate that $\mathrm{Fam}_{3} \mathrm{~b}^{-/-}$osteoblasts have impaired proliferation compared with wild-type cells.

These data demonstrate Fam73b is necessary for skeletal growth and normal bone structure and strength and suggest that a novel pathway involving mitochondrial fusion and function in osteoblasts is required for the establishment and maintenance of bone mass.

\section{P22}

Independent skeletal phenotyping of Creb3I1 knockout mice confirms validity of signature in human GWAS

\author{
Elena J Ghirardello, ${ }^{1}$ John G Logan, ${ }^{1}$ Penny C Sparkes, ${ }^{1}$ \\ Katherine F Curry, ${ }^{1}$ Justyna J Miszkiewicz, ${ }^{1}$ Victoria D \\ Leitch, ${ }^{1}$ Natalie C Butterfield, ${ }^{1}$ Peter I Croucher, ${ }^{3}$ Graham $\mathbf{R}$ \\ Williams, ${ }^{1}$ JH Duncan Bassett, ${ }^{1}$ and Sanger Mouse Pipelines ${ }^{2}$ \\ ${ }^{1}$ Molecular Endocrinology Group, Imperial College London, \\ London, UK \\ ${ }^{2}$ Sanger Mouse Pipelines, Wellcome Trust Sanger Institute, \\ Cambridge, UK \\ ${ }^{3}$ Garvan Institute of Medical Research, Sydney, Australia
}

A meta-analysis of 30 genomewide association studies (GWAS) identified that $C R E B 3 L 1$ is associated with bone mineral density (BMD). CREB3L1 (CAMP-responsive element-binding protein 3like protein 1) is involved in the unfolded protein response to endoplasmic reticulum stress, the regulation of type 1 collagen expression, and has been implicated in bone development, osteopenia, and fracture repair. The CREB3L 1 locus, which also harbors ARHGAP1 and LRP4, has previously been associated with $B M D$, but the causative gene explaining the GWAS signal is unknown.

The Origins of Bone and Cartilage Disease (OBCD) project is undertaking rapid-throughput skeletal phenotyping of knockout mice generated by the International Knockout Mouse Consortium to identify novel genetic determinants of bone mass and strength. Bone mineral content (BMC) and morphology of the femur and caudal vertebrae were determined by X-ray microradiography, structural parameters of trabecular and cortical bone by micro-computed tomography, and bone strength parameters by biomechanical testing. Femur and vertebra samples from 16 -week-old female $\mathrm{Creb} 3 / 1^{-/-}$knockout mice were analyzed in comparison to reference data obtained for all parameters from >300 age-, sex-, and background- 
matched wild-type mice. Results that differed from the reference range mean by greater than 2 SD were identified as statistically significant outliers.

Creb3/1/ mice had $9 \%$ and $13 \%$ decreases in femoral and vertebral BMC, with trabecular bone volume decreased by $44 \%$ and cortical thickness decreased by $22 \%$. These structural abnormalities resulted in markedly impaired bone strength; the maximum femoral load was decreased by $51 \%$ ( $>5$ SD), while the maximum vertebral load under compression was reduced by $60 \%$ ( $>5$ SD). By contrast, the skeletal phenotype of heterozygous $\mathrm{Creb} 3 / 1^{+/-}$mice was normal.

These data provide functional validation of the association between CREB3L1 and BMD identified by GWAS meta-analysis and suggest a causative role for $C R E B 3 L 1$ in the pathogenesis of osteoporosis and fracture susceptibility.

\section{P23}

Osteocalcin regulates arterial calcification via altered Wnt signalling and glucose metabolism

\section{Nabil Rashdan, ${ }^{1}$ Alisia Sim, ${ }^{2}$ Peter Hohenstein, ${ }^{1}$ John Hung, ${ }^{3}$ Jakub Kaczynski, ${ }^{3}$ David Newby, ${ }^{3}$ Andrew Baker, ${ }^{3}$ Gerard Karsenty, ${ }^{4}$ and Vicky MacRae ${ }^{1}$}

\author{
${ }^{1}$ The Roslin Institute and Royal (Dick) School of Veterinary Studies, \\ University of Edinburgh, Edinburgh, UK \\ ${ }^{2}$ School of Chemistry, University of Edinburgh, Edinburgh, UK \\ ${ }^{3}$ Centre for Cardiovascular Science, University of Edinburgh, \\ Edinburgh, UK \\ ${ }^{4}$ Department of Genetics and Development, Columbia University, \\ New York, USA
}

Objective: Arterial calcification (AC) is a strong independent predictor of cardiovascular mortality and shares many similarities to skeletal mineralization. This study addressed the hypothesis that ablation of the osteoblast-specific protein osteocalcin (OCN) reduces $A C$.

Methods: Calcification of rat aortic rings and $\mathrm{OCn}^{-/-}$and wildtype (WT) murine vascular smooth muscle cells (VSMCs) was induced by $3 \mathrm{mM}$ phosphate (Pi). Calcium deposition was determined using Alizarin red staining and a modified ocresolphthalein method. Immunohistochemistry (IHC) was performed on 5 uM human carotid artery plaque sections. Gene expression was analyzed by qRT-PCR. VSMCs were cultured with 2-(N-(7-Nitrobenz-2-oxa-1,3-diazol-4-yl)Amino)-2Deoxyglucose (2-NBDG) to determine glucose uptake.

Results: Calcified VSMCs showed increased Ocn mRNA expression (1.73-fold; $p<0.001$ ). IHC revealed OCN co-localization with calcification areas in human and rat arterial sections.

Decreased calcium deposition was observed in $\mathrm{Ocn}^{-1-}$ VSMCs compared with WT $(63 \%$ decrease, $p<0.001)$. $\mathrm{Ocn}^{-/-}$VSMCs showed reduced mRNA expression of the osteogenic markers Runx2 (0.51-fold, $p<0.01$ ), and PiT1 (0.70-fold, $p<0.001$ ), with a concomitant increase in the calcification inhibitor Mgp compared with WT (1.42-fold, $p<0.05)$.

Wnt signalling can impact AC. Ocn ${ }^{-/-}$VSMCs showed reduced mRNA expression of Wnt signalling markers Axin2 (0.13-fold, $p<$ $0.001)$ and cyclin $D(0.71$-fold, $p<0.01)$. Conversely, $D k k 1$ expression was increased (1.55-fold, $p<0.05)$. Treatment with CHIR99021 (Wnt activator) increased calcium deposition in WT and $\mathrm{Ocn}^{-/-}$VSMCs $(1 \mu \mathrm{M} ; \mathrm{p}<0.001) . \mathrm{Ocn}^{-/-}$VSMCs, however, calcified less than WT ( $1 \mu \mathrm{M} ; 0.27$-fold, $p<0.001)$.
Consistent with the role of OCN in glucose utilization, $\mathrm{OCn}^{-1}$ VSMCs exhibited reduced glucose uptake compared with WT (0.38-fold, $p<0.05)$. This was accompanied by reduced mRNA expression of Glut1 (0.78-fold, $p<0.001$ ), Hex1 (0.77-fold, $p<$ $0.01)$, and Pdk4 (0.47-fold, $p<0.001)$. Dichloroacetate (DCA), a PDK inhibitor that increases PDH activity, decreased VSMC calcification in WT cells $(1 \mathrm{mM} ; p<0.05)$. Furthermore, DCA attenuated the effects of CHIR99021 (1 uM) on calcium deposition $(p<0.05)$.

Conclusion: These data suggest that the pro-calcific effects of OCN may be dependent on Wnt signalling and mediated by glucose metabolism.

\section{P24}

The regulation of bone mineralization in in vitro and in vivo models of chronic kidney disease

\author{
Shun-Neng Hsu, ${ }^{1}$ Vicky MacRae, ${ }^{1}$ Amanda Novak, ${ }^{1}$ \\ Katherine Staines, ${ }^{2}$ and Colin Farquharson ${ }^{1}$ \\ ${ }^{1}$ The Roslin Institute, University of Edinburgh, Edinburgh, UK
${ }^{2}$ School of Applied Sciences, Edinburgh Napier University,
Edinburgh, UK
}

Objectives: Patients with chronic kidney disease (CKD) encounter complex bone and mineral abnormalities resulting in bone fractures. Although PHOSPHO1 and tissue non-specific alkaline phosphatase (TNAP) activity are essential for bone mineralization, their function and molecular mechanisms in CKD bone remain incompletely understood. The aim of this study was to examine the effects of increased PTH, FGF-23, and phosphate $(\mathrm{Pi})$, all elevated in $\mathrm{CKD}$, on $\mathrm{PHOSPHO} 1$ and TNAP expression.

Methods: The MC3T3 osteoblast cell line was cultured for 6 days with increasing concentrations of PTH (0.05-50 nM), FGF-23 (1$200 \mathrm{ng})$, and $\mathrm{Pi}(2-5 \mathrm{mM})$ for the last 24 hours. Matrix mineralization was assessed by Alizarin red staining, and gene and protein expression by RT-qPCR and Western blotting, respectively. We also established the adenine model of CKD in 10 -week-old male mice via dietary $0.2 \%$ adenine supplementation for up to 6 weeks. Biochemical analysis was performed on urine and blood.

Results: PTH (50 nM) induced a significant decrease in Phospho1 expression ( $p<0.001)$, as did increasing concentrations of FGF23 ( $p<0.05)$. Conversely, low levels of Pi significantly increased Phospho1 expression $(p<0.001)$. These data were confirmed by Western blotting. The expression of Alpl was reduced by increasing concentrations of PTH, FGF-23, and Pi $(p<0.01)$. Moreover, higher $\mathrm{Pi}(5 \mathrm{mM})$ significantly increased matrix mineralization $(p<0.01$ ), while FGF-23 decreased it (not significant). In the CKD mouse model, blood levels of urea nitrogen (81.2 versus $29.8 \mathrm{mg} / \mathrm{dL}, p<0.05)$ and creatinine $(0.52$ versus $0.32 \mathrm{mg} / \mathrm{dL}, p<0.001$ ) were significantly higher in comparison to control groups. Similar trends were observed in urine samples. Ongoing studies will determine systemic hormone profiles and bone expression levels of PHOSPHO1 and TNAP.

Conclusion: This study has revealed that PHOSPHO1 and TNAP may participate in the molecular pathogenesis underlying the bone mineralization in CKD. We have also described the feasibility of developing a model of CKD mice to evaluate associations between CKD and bone mineralization. 


\section{All that fractures is not bone: microscopic anatomy of vertebral bodies}

\section{Alan Boyde ${ }^{1}$ and David Mills ${ }^{1}$}

Biophysics OGD, Queen Mary University of London, London, UK

Objective: To understand the interface between cortical shell and cancellous bone in human vertebral bodies, age changes, and probable mechanical significance.

Methods: Archival material, 3- to 4-mm mid-body vertical slices, 80 L2 embedded PMMA: blocks polished, carbon coated, $20 \mathrm{kV}$ qBSE SEM; high-contrast resolution X-ray microtomography (XMT: 44 hour scans); iodine vapor staining and further BSE SEM, uncoated. Some 10- $\mu \mathrm{m}$ laser ablation machined sections from block surfaces for polarized light microscopy (PLM). 50 L4 macerated for 3D BSE SEM.

Results: Mineral concentration: Cortex contains lamellar bone and more highly mineralized tissues: ligament, dense fibrous periosteum, or Sharpey fiber bone.

2D SEM with iodine staining, PLM: Uncalcified osteoid, ligament, fibrous periosteum, and Sharpey fibers can be distinguished. 3D SEM: Inimitable branching bundle morphology of bone collagen lamellae was displayed on all (re)modelled surfaces of trabeculae and endocortex, modified where penetrated by any Sharpey fibers at ectocortex. However, the greatest part of the most exterior cortex is composed of strictly parallel, nonbranching collagen, either mostly longitudinal-ligament-or decussating layers of dense fibrous periosteum.

Ligament tissue becomes incorporated in the bone organ by calcification extending into it from the mineralizing front of bone tissue proper. Owing to endocortical resorption, sections of the entire shell thickness can be composed of non-bone.

Calcified tissues in vertebral cortical shells include matrices that are, emphatically, not bone: They have a different structure, their collagen is not made by osteoblasts, and generally reach a higher level of mineralization than bone; they will be assessed as "thicker bone" with CT. Further, these phases cannot be recognized from bone by clinical imaging, and it is highly improbable that they will be distinguished using decalcification and staining LM histology.

If lamellar structure is ideal, then the anterior cortex in particular is not.

Conclusion: The proportion of calcified ligament tissue masquerading as bone dramatically increases in anterior collapse, often in hand with thickening of this cortex. It remains to be elucidated whether failure is favored by this less auspicious and perhaps more fragile structure. A vicious circle?

\section{P26}

Inhibition of the protein kinase $R$ signaling pathway in vivo reduces bone remodeling in post-traumatic osteoarthritis

\section{Sophie Gilbert, Cleo Bonnet, Menna Ihenacho, Rose-Marie Cronin, Emma Blain, and Debbie Mason}

\section{School of Biosciences, Cardiff University, Cardiff, UK}

Objectives: Protein kinase $\mathrm{R}$ (PKR) is important in proinflammatory cytokine signaling pathways and is implicated in the pathogenesis of arthritis. We have shown that knockout of the endogenous inhibitor of PKR resulting in overactivation of the pathway in mice results in osteophyte formation, bone thickening, and calcification of the collateral ligaments within the medial knee compartment. Phosphorylated PKR is also evident in the inflammatory infiltrate and newly forming osteophytes in post-traumatic osteoarthritis (PTOA). This study investigates the role of PKR in bone remodeling in an anterior cruciate ligament $(A C L)$ rupture model of PTOA.

Methods: Right knees of anesthetised, 12-week-old C57Bl6 mice were loaded once at $12 \mathrm{~N}$ to rupture the $\mathrm{ACL}$. Mice received an intra-articular injection of the PKR inhibitor (PKRi; 22 or $222 \mu \mathrm{M}$ ) or carrier (DMSO; $0.05 \%$ or $0.5 \%$ ) immediately after $A C L$ rupture. Contralateral (no load, no injection) and unloaded (+injection) knees served as controls. Mice were culled at 21 days and knees processed for histology. Sections were scored for bone changes and osteophyte formation using the OARSI system.

Results: Injection of DMSO (0.5\%) alone resulted in a 6.4-fold increase in bone remodeling compared with contralateral knees $(p=0.048)$ and caused an inflammatory cell infiltrate. Injection of PKRi at $222 \mu \mathrm{M}$ (DMSO $0.5 \%)$ reduced this $(p=0.05)$. ACL rupture resulted in an increase in bone remodeling $(p=0.001)$, which was not prevented by PKRi at $222 \mu \mathrm{M}$. However, PKRi at $22 \mu \mathrm{M}$ (DMSO $0.05 \%$ ) reduced the extent of bone changes $(p=0.047)$, which was most apparent in the lateral compartment.

Conclusion: Intra-articular injection of DMSO alone caused significant thickening of the subchondral bone and induced osteophyte formation and inflammatory cell infiltrate; PKRi prevented this, suggesting a role for PKR signaling in bone remodeling. Inhibition of PKR also reduced the load-induced bone changes but only when the level of DMSO was reduced to $0.05 \%$. This data suggests that inhibition of the PKR pathway may provide a therapy for the treatment of bone changes in PTOA.

\section{P27}

Bone geometry is correlated with oscillometric arterial stiffness in overweight older adults with low vitamin D

\author{
Alexander Rodriguez, ${ }^{1}$ Cecilia $\mathrm{Xu},{ }^{1}$ Lachlan McMillan, ${ }^{1}$ \\ Velandai Srikanth, ${ }^{2}$ David Scott, ${ }^{1}$ and Peter Ebeling ${ }^{1}$ \\ ${ }^{1}$ Bone and Muscle Health Research Group, School of Clinical \\ Sciences, Monash University, Melbourne, Australia \\ ${ }^{2}$ Stroke and Aging Research Group, Frankston Hospital, Monash \\ University, Melbourne, Australia
}

Background: Vitamin D deficiency and adiposity have deleterious effects on skeletal health and can increase arterial stiffness. Additionally, low bone mass is associated with increased arterial disease, but little is known about the association of bone geometry with arterial disease.

Objective: To determine the association between bone geometry and arterial stiffness in overweight (body mass index $\left.>25 \mathrm{~kg} / \mathrm{m}^{2}\right)$, older adults (50-80 years) with low vitamin $\mathrm{D}(<50$ $\mathrm{nmol} / \mathrm{L}$ ) who were recruited to a randomized controlled trial investigating the effects of vitamin $D$ and exercise on these outcomes.

Methods: Cross-sectional analysis at baseline. Demographics and clinical history were obtained via questionnaires and anthropometrics determined using standard protocols. Tibial bone geometry at the $4 \%$ and $66 \%$ sites was assessed via 
peripheral quantitative computed tomography (XCT3000, Stratec, Germany). Blood pressure and measures of arterial stiffness were determined by an oscillometric device (Mobil-oGraph, IEM, Germany). These measures included augmentation index (proportion of blood pressure waveform attributable to the reflection wave) and pulse pressure (difference between systolic and diastolic blood pressure).

Results: Thirty individuals were recruited (women: $n=15$ [50\%]). Mean systolic blood pressure was high-normal $(128.6 \pm 13.5 \mathrm{mmHg})$. In age- and BMl-adjusted models, there was an inverse correlation between trabecular area at $4 \%$ site $(-0.799 ; p<0.001)$; trabecular volumetric bone mineral density $(-0.578 ; p=0.003)$; cortical area at $66 \%$ site $(-0.549 ; p=0.008)$; fracture $\operatorname{load}(\mathrm{x})(-0.465 ; p=0.028)$; fracture $\operatorname{load}(\mathrm{y})(-0.693 ; p<$ 0.001 ) with augmentation index. Additionally, there was an inverse correlation between trabecular area at $4 \%$ site $(-0.416$; $p=0.027)$ and cortical area at $66 \%$ site $(-0.449 ; p=0.018)$ with pulse pressure. Bone marrow density correlated positively with pulse pressure $(0.448 ; p=0.021)$. In regression analyses adjusting for age, BMI, and sex, trabecular area was inversely associated with augmentation index $(\beta=-5.666$; $95 \%$ confidence interval $-8.807,-2.426$ ).

Conclusion: Poorer bone geometry was correlated with augmentation index, providing further evidence for an association between bone loss and vascular disease suggestive that treatments that improve skeletal health may have favorable effects on arterial stiffness. However, it remains uncertain if resolving underlying adiposity and vitamin $D$ deficiency in this cohort may improve these outcomes.

\section{P28}

VEGF exerts sexually dimorphic effects on bone mass and architecture

\section{Alice Goring, ${ }^{1}$ Behzâd Javaheri, ${ }^{2}$ Napoleone Ferrara, ${ }^{3}$ Bjorn Olsen, ${ }^{4}$ Philipp Schneider, ${ }^{5}$ Richard Oreffo, ${ }^{6}$ Andrew Pitsillides, $^{2}$ and Claire Clarkin ${ }^{1}$}

\author{
${ }^{1}$ Biological Sciences, University of Southampton, Southampton, UK \\ ${ }^{2}$ Comparative Biomedical Sciences, Royal Veterinary College, \\ London, UK \\ ${ }^{3}$ Genetech, San Francisco, CA, USA \\ ${ }^{4}$ Department of Cell Biology, Harvard School of Dental Medicine, \\ Boston, MA, USA \\ ${ }^{5}$ Faculty of Engineering and the Environment, University of \\ Southampton, Southampton, UK \\ ${ }^{6}$ Faculty of Medicine, University of Southampton, Southampton, \\ UK
}

Objectives: Vascular Endothelial Growth Factor (VEGF) is required for the tight coupling of osteogenesis and angiogenesis, and deletion of VEGF in early osteoblast (OB) cells results in an osteoporotic phenotype ${ }^{(1)}$ and diminished fracture repair. ${ }^{(2)}$ Similarly, we have recently found that loss of VEGF in mature osteocalcin-expressing cells results in an increase in cortical porosity in adult and aged mice, which is more pronounced in males (2.7-fold, $p<0.001)$ than females $(0.54$-fold, $p<0.01)$. The aim of this study was to examine this sexual dimorphism further by measuring structural and architectural parameters after $O B$ VEGF deletion.

Methods: For VEGF knockout OBs (OBVEGFKO), 16-week-old male and female mice carrying floxed alleles of VEGF and expressing Cre recombinase under the control of the osteocalcin promotor were used $(n=4)$. Tibias were scanned using Skyscan 1172 at $18 \mu \mathrm{m}$ voxel size. Whole bone analyses were performed using BoneJ. ${ }^{(3)}$

Results: Whole tibia analysis reveals that VEGF deficiency leads to significant reduction in bone mass: cross-sectional area and mean thickness at many locations along the entire tibia in female OBVEGFKO versus controls. No significant effect of VEGF deficiency was found on any of these parameters in male OBVEGFKO versus controls. Our data also show that Imin and Imax are significantly reduced in midshaft and toward distal tibia in female but not male OBVEGFKO compared with controls. The sexual dimorphic effect of VEGF deficiency is reflected in reduction in predicted resistance to torsion and alteration to tibial ellipticity in female OBVEGFKO only.

Conclusion: Our data reveal a sex-specific role for VEGF in modulating bone mass and architecture. They suggest that osteoblast-derived VEGF contributes to the pathogenesis of osteoporosis and that therapeutic strategies for osteoporosis should be cognizant of the sexual dimorphic role of VEGF in controlling bone architecture and mass.

Funded by Arthritis Research UK.

\section{References}

1. Liu Y, Berendsen AD, Jia S, et al. Intracellular VEGF regulates the balance between osteoblast and adipocyte differentiation. J Clin Invest. 2012;122(9):3101-13.

2. Hu K, Olsen BR. Osteoblast-derived VEGF regulates osteoblast differentiation and bone formation during bone repair. J Clin Invest. 2016;126(2):509-26.

3. Doube M, Klosowski MM, Arganda-Carreras I, et al. Bone. 2010;47(6):1076-9.

\section{P29}

Demonstrating Fracture Liaison Service effectiveness-use of fracture incidence and prescribing data to demonstrate clinical and cost effectiveness in a small population

\section{Tim Jones}

Service Development, National Osteoporosis Society, Bath, UK

Objectives: The National Osteoporosis Society (NOS) Service Development Team supports Fracture Liaison Services (FLS) in various ways, including by demonstrating to payors the effectiveness of services in preventing secondary fractures. Health and social care payors typically use crude, populationlevel hip fracture rates to evaluate services, even though these comprise less than 1 in 5 of prevented fractures and include primary and non-osteoporotic fractures. Alternative indicators of effectiveness are needed such as the number and rate of patients on anti-osteoporosis medication (AOM). This analysis sought to demonstrate whether publicly available prescribing data could be used to develop an indicator of FLS effectiveness. Methods: An analysis was conducted of AOMs dispensed to patients in England over 48 months. These data were adjusted and standardized to output a rate of patients on treatment per 1000 population aged 50 years and above (PoTr). The data were analyzed to understand national trends in the use of AOMs and to explore whether the PoTr was a useful measure of effectiveness of FLS in a given population.

Results: The analysis showed a downward trend in the number and rate of patients on treatment over the study period 
consistent with international studies. In some case studies, the establishment or disestablishment of an FLS was observed to be statistically significantly related to changes in the rate of patients on treatment.

\section{P30}

Falls risk is increased and bone mineral density reduced in individuals with rheumatoid arthritis: findings from UK biobank

\section{Michael Clynes, ${ }^{1}$ Karen Jameson, ${ }^{1}$ Daniel Prieto-Alhambra, ${ }^{2}$ Nicolas Harvey, ${ }^{1}$ Cyrus Cooper, ${ }^{1,3}$ and Elaine Dennison ${ }^{1}$ \\ ${ }^{7}$ MRC Lifecourse Epidemiology Unit, University of Southampton, Southampton, UK \\ ${ }^{2}$ Centre for Statistics in Medicine, University of Oxford, Oxford, UK ${ }^{3}$ NIHR Musculoskeletal Biomedical Research Unit, University of Oxford, Oxford, UK}

Objectives: Rheumatoid arthritis (RA) is known to be associated with adverse musculoskeletal outcomes. The current study aimed to explore if RA is a predictor of reduced bone mineral density and falls, using baseline data from the UK Biobank cohort.

Methods: The study population consists of 500,954 people aged 37 to 73 years. A diagnosis of RA was identified in participants from the UK Biobank using Hospital Episode Statistics using ICD10 codes M05 and M06. Multivariable logistic and linear regressions were used to assess the relationships with estimated bone mineral density (eBMD) as assessed by heel ultrasound and with falls (occurring in the year before baseline interview and recorded by self-report).

Results: Of 500,954 participants (median age 58 years [IQR 5063]), 5476 (1.1\%) were found to have a diagnosis of RA. A total of 321,904 participants had heel ultrasound measurements performed, generating a mean eBMD $T$-score of -0.32 . Data were available for self-reported falls in 499,956 participants, of which a total of $99,120(19.8 \%)$ had fallen in the last year, with 65,968 (13.2\%) having had just one fall and 33,152 (6.6\%) having had more than one fall. A diagnosis of RA was positively associated with having a lower eBMD (mean diff. $T$-score -0.346 , $95 \% \mathrm{Cl}-0.386,-0.306, p<0.01$, adjusted for sex). Furthermore, individuals with a diagnosis of RA were also significantly more likely to report a fall in the last year than those without ( $\mathrm{OR}=2.01,95 \% \mathrm{Cl} 1.90,2.13, p<0.01$, adjusted for sex). These observations remained significant after adjustment for potential confounders (age, ethnicity, BMI, smoker status, and activity).

Conclusion: Rheumatoid arthritis was associated with lower eBMD and an increased likelihood of falls in this cross-sectional analysis. These data may help inform future strategies for reducing fracture risk in individuals with RA as both low eBMD and falls are independent risk factors for fracture. This research has been conducted using the UK Biobank Resource.

\section{P31}

Identification of vertebral fractures in Fracture Liaison Services (FLS) in the UK

\section{Sonya Stephenson}

National Osteoporosis Society, Bath, UK
Objective: Fracture Liaison Services (FLS) prevent secondary fractures through systematic identification of fragility fractures using case finding, with assessment and treatment of osteoporosis where necessary. Services are measured for quality against the National Osteoporosis Society (NOS) Clinical Standards for FLS. Standard 1 asserts that all patients over 50 years with a newly reported vertebral fracture will be systematically and proactively identified. This analysis sought to establish to what extent this standard is being met in the UK.

Methods: A rolling gap analysis of FLS provision for identification of vertebral fractures in patients aged over 50 years has been undertaken by the NOS Service Development team. This measures service provision against the national standard. Data were collected at 110 sites across the UK between 2014 and 2018.

Results: Seventy-seven percent (85) of sites had no systematic process in place to identify vertebral fractures. Only $8 \%$ (9) sites identified all newly reported vertebral fractures. Fifteen percent (16) had procedures in place to identify some vertebral fractures, ie, within certain cohorts. There was considerable disparity across the UK. Sites in Scotland were significantly more likely to have comprehensive processes in place $(38 \%, 6 / 16)$ than in the rest of the UK (3\%, 3/94).

Conclusion: Gap analysis shows a paucity of provision for systematic identification of vertebral fractures. Vertebral fracture identification is particularly challenging because most patients present with back pain in primary care, are treated with analgesics, and do not have their fracture identified. In secondary care, patients present across a number of departments (Rheumatology, Orthopedic, Fracture Clinic, A\&E, Spinal services). The most effective approach therefore involves commitment from Diagnostic Imaging to identify vertebral fractures and report them unambiguously. Referring clinicians should be alerted to vertebral fractures identified incidentally, with clear protocols in place for onward management, or referral to FLS.

\section{P32}

IRF5 is required for macrophage-driven bone formation

\section{Adel Ersek, Carlotta Cosulich, Irina Udalova, and Nicole Horwood}

Kennedy Institute of Rheumatology, University of Oxford, Oxford, UK

The contribution of excessive inflammation to osteoclastogenesis and bone loss is well documented. Conversely, inflammation is a critical component of bone formation during fracture repair, and macrophages can contribute to osteoblast differentiation both in vitro and in vivo. We and others have shown that oncostatin $M$ produced by macrophages is capable of promoting bone formation; however, the exact types of macrophages involved in the bone anabolism remain to be described.

The transcription factor interferon regulatory factor 5 (IRF5) is the master regulator of inflammatory macrophage polarization. Using micro-CT, we investigated the baseline phenotype of mice deficient for IRF5 and their wild-type (WT) littermates from 6 to 24 weeks of age and did not observe any significant differences in bone density and architecture. Next, 8-week-old female mice (WT and IRF5-/-) underwent ovariectomy-induced osteoporosis. 
After a further 8 weeks, the tibia and vertebrae were analyzed by micro-CT; however, once again there was no statistically significant difference between the two groups; both lost an equivalent amount of bone mass. Thirdly, we investigated the effect of low-dose LPS $(20 \mu \mathrm{g})$ on calvarial bone formation. Highdose LPS $(500 \mu \mathrm{g})$ induces osteolytic lesions in the calvaria via osteoclast formation; however, we have found that lower doses of LPS are bone anabolic. IRF5-/- and WT mice were challenged with a single dose of LPS $(20 \mu \mathrm{g})$ and left for a further 14 days before euthanization. WT mice showed a significant increase in BV/TV and mineral apposition rate, whereas IRF5-/- mice did not have a bone anabolic response to LPS. In vitro co-cultures of murine mesenchymal stem cells with macrophages derived from either IRF5-/- or WT showed that the WT macrophages were able to promote osteoblast formation in the presence of LPS, whereas the IRF5-/- macrophages failed to do so, confirming that it is a direct effect of inflammatory macrophages on osteoblast formation that accounts for the increase in bone formation observed in vivo.

This work highlights the importance of ensuring that therapeutic interventions do not completely ablate inflammation as it is a central and critical component to bone formation after traumatic bone injury.

\section{P33-Abstract withdrawn}

\section{P34}

Relationships between muscle size, strength, and function and the risk of falls and fractures

\author{
Nicholas Fuggle, ${ }^{1}$ Karen Jameson, ${ }^{1}$ Mark Edwards, ${ }^{1,2}$ Elaine \\ Dennison, ${ }^{1}$ and Cyrus Cooper ${ }^{1,3}$ \\ ${ }^{1}$ MRC Lifecourse Epidemiology Unit, University of Southampton, \\ Southampton, UK \\ ${ }^{2}$ Rheumatology Department, Portsmouth Hospitals NHS Trust, \\ Portsmouth, UK \\ ${ }^{3}$ NIHR Musculoskeletal Biomedical Research Unit, University of \\ Oxford, Oxford, UK
}

Objectives: Sarcopenia is thought to be an important contributor to falls risk and hence fractures. The aim of this study was to examine the sex-related associations between falls and fractures and muscle measures of mass, strength, and function in a population-based cohort of older adults.

Methods: A sample of 642 participants of the Hertfordshire Cohort Study (community-dwelling, older adults) were recruited. Muscle mass was assessed as muscle cross-sectional area by peripheral quantitative computed tomography of the calf; muscle strength was assessed by dynamometry, and muscle function was measured using gait speed. Data on falls and fractures were collected via questionnaire, which was repeated after 5 to 7 years. Logistic regression was used to examine associations between muscle measures and outcomes with and without adjustment for age, body mass index, social class, smoker status, alcohol consumption, activity level, calcium intake, hormone replacement therapy use, and years since the menopause in women.

Results: The mean (SD) ages at baseline were 69.2 (2.5) and 69.5 (2.6) years for men and women, respectively. Falls since the age of 45 and in the last year at baseline and incident fractures and falls at follow-up were significantly more common in women $(p<0.05)$.
Greater calf muscle area (Z-score) was associated with fewer falls in women in the last year at baseline $(\mathrm{OR}=0.64,95 \% \mathrm{Cl} 0.43,0.95$, $p=0.03$ after adjustment), but no statistically significant relationships were found in men or with longitudinal analyses. Higher grip strength (Z-score) at baseline was negatively associated with having a fracture since the age of $45(\mathrm{OR}=0.74,95 \% \mathrm{Cl} 0.56,0.99, p$ $<0.05)$ but not lower risk of falls since baseline $(\mathrm{OR}=1.50,95 \% \mathrm{Cl}$ $1.07,2.10, p<0.02$ ) in women in adjusted models. Higher baseline gait speed (Z-score) was associated with fewer falls in the last year at follow-up in men (OR $=0.65,95 \% \mathrm{Cl} 0.43,0.99, p<0.05)$ but not women.

Conclusion: We saw different relationships between muscle mass, strength, and function with falls and fractures in men and women; function was more important in men, and size in women. Further studies are now required to reproduce these results.

\section{P35}

Does modeling-based bone formation continue on trabecular surfaces throughout life? A histological analysis of forming minimodeling structures in the human femoral head

Hiroshige Sano, ${ }^{1,2,3}$ Naoki Kondo, ${ }^{1}$ Taketoshi Shimakura, ${ }^{2}$ Junichi Fujisawa, ${ }^{1}$ Yasufumi Kijima, ${ }^{1}$ Linda Skingle, ${ }^{3}$ Kenneth Poole, ${ }^{3}$ Noriaki Yamamoto, ${ }^{2,4}$ Hideaki Takahashi, $^{2,4}$ and Naoto Endo ${ }^{1}$

${ }^{1}$ Orthopedic Surgery, Niigata University, Niigata, Japan

${ }^{2}$ Niigata Bone Science Institute, Niigata, Japan

${ }^{3}$ Medicine, University of Cambridge, Cambridge, UK

${ }^{4}$ Niigata Rehabilitation Hospital, Niigata, Japan

Bone modeling is a biological process of bone formation that adapts bone size and shape to mechanical loads, especially during childhood and adolescence. Bone modeling in cortical bone can be easily detected using sequential radiographic images, while its assessment in trabecular bone is challenging. Here we performed histomorphometric analysis in 21 bone specimens from biopsies collected during hip arthroplasty, and we proposed the criteria for histologically identifying an active modeling-based bone formation, which we call a "forming minimodeling structure" (FMiS).

A bone block was excised from the central region of the femoral head. Villanueva bone staining was performed and embedded in methylmethacrylate without decalcification. We proposed the following histological criteria for the definition of the FMiS: a) the base of the FMiS is a smooth lamellar bone surface; b) the lamellar pattern of the FMiS is different from that of the base of the bone surface; $c$ ) the surface of the FMiS is covered with an osteoid seam; and d) the degree of fluorescent signal in bone in the FMiS is lower than that of older adjacent bone.

Evidence of FMiSs was found in 9 of 20 specimens (45\%). In histomorphometric analysis, bone volume was significantly higher in specimens displaying FMiSs compared with the specimens without these structures (BV/TV, 31.7\% \pm 10.2 versus $23.1 \pm 3.9 ; p<0.05)$. Osteoid parameters were raised in FMiScontaining bone specimens (OV/BV, $2.1 \pm 1.6$ versus $0.6 \pm 0.3 \%$; $p<0.001$, OS/BS, $23.6 \% \pm 15.5$ versus $7.6 \pm 4.2 ; p<0.001$, and $\mathrm{O}$. Th, $7.4 \mu \mathrm{m} \pm 2.0$ versus $5.2 \pm 1.0 ; p<0.05$ ).

Our results showed that modeling-based bone formation on trabecular bone surfaces occurs even during adulthood. As 
FMiSs can represent histological evidence of modeling-based bone formation, understanding of this physiology in relation to bone homeostasis is crucial.

\section{P36}

An investigation of polymethylmethacrylate bone cement loaded with amoxicillin encapsulated in liposomes

\section{Rebecca Beamish, ${ }^{1,2}$ Wayne Ayre, ${ }^{3}$ and Sam Evans ${ }^{2}$}

${ }^{1}$ School of Engineering, Cardiff University, Cardiff, UK

${ }^{2}$ Trauma and Orthopaedics, Basingstoke Hospital, Basingstoke, UK

${ }^{3}$ School of Dentistry, Cardiff University, Cardiff, UK

Objectives: Investigate the incorporation of an antibiotic in bone cement using liposomes (a drug delivery system) with the potential to promote osseointegration at the bone cement interface while maintaining antibiotic elution, anti-microbiological efficacy, and cement mechanical properties.

Prosthetic joint infection and aseptic loosening are associated with significant morbidity. ${ }^{(1)}$ Antibiotic loaded bone cement is commonly used and successfully reduces infection rates; however, there is increasing resistance to the commonly used gentamicin. ${ }^{(2,3)}$

Previous studies have shown gentamicin incorporated into bone cement using liposomes can maintain the cement's mechanical properties and improve antibiotic elution. ${ }^{(3)}$

The phospholipid phosphatidyl-I-serine has been postulated to encourage surface osteoblast attachment ${ }^{(4)}$ and in a liposome could improve osseointegration, thereby reducing aseptic loosening.

Preliminary clinical isolate testing showed excellent antimicrobial action with amoxicillin, therefore the study aims were to test amoxicillin incorporated into bone cement using liposomes containing phosphatidyl-I-serine in terms of antibiotic elution, microbiological profile, and mechanical properties.

Methods: Amoxicillin was encapsulated within $100 \mathrm{~nm}$ liposomes containing phosphatidyl-L-serine and added to PMMA bone cement (Palacos R, Heraeus Medical, Newbury, UK).

Mechanical testing was performed according to Acrylic Cement standards (ISO BS 5833:2002). ${ }^{(5)}$ Elution testing was carried out along with microbiological testing utilizing clinical isolates.

Results: Liposomal encapsulated amoxicillin PMMA bone cement exceeded minimum ISO BS 5833:2002 standards and had better elution when compared with plain amoxicillin or commercial gentamicin cement (Palacos $\mathrm{R}+\mathrm{G}$, Heraeus Medical, Newbury, UK).

Amoxicillin showed superior antimicrobial action when compared with gentamicin of the same concentration. However, liposomal encapsulated amoxicillin in solution and liposomal encapsulated amoxicillin in PMMA were both less effective than free amoxicillin in bacterial growth inhibition. The liposomal amoxicillin also seemed to decrease the cement setting time.

Conclusion: Amoxicillin is a credible alternative to gentamicin for incorporation in PMMA bone cement. It has shown superior antibacterial action, which may improve infection rates, while maintaining the cement's mechanical properties. PhosphatidylI-serine containing liposomes maintained the cement's mechanical properties, however, had less effective antibacterial action. This difference in antibacterial action requires further investigation along with investigation of osteoclast attachment to phosphatidyl-I-serine containing liposomes within cement.

\section{References}

1. National Joint Registry. National Joint Registry for England, Wales and Northern Ireland (NJR), 14th Annual Report 2017, Surgical data to 31 December 2016. 2017. Available at: www. njrcentre.org.uk. Accessed October 10, 2017.

2. Corona PS, Espinal L, Rodriguez-Pardo D, Pigrau, Larrosa N, Flores $\mathrm{X}$. Antibiotic susceptibility in gram-positive chronic joint arthroplasty infections: increased aminoglycoside resistance rate in patients with prior aminoglycoside-impregnated cement spacer use. J Arthoplasty. 2014;29(8):1617-21.

3. Ayre WN, Birchall JC, Evans SL, Denyer SP. A novel liposomal drug delivery system for PMMA bone cements. J Biomed Mater Res B Appl Biomater. 2016;104(8):1510-24.

4. Merolli $A$, Bosetti $M$, Giannotta $L$, et al. In vivo assessment of the osteointegrative potential of phosphatidylserine-based coatings. J Mater Sci Mater Med. 2006;17(9):789-94.

5. British Standards Institution. British Standard: BS ISO 5833:2002 Implants for surgery-acrylic resin cements. British Standards Institution, June 2002.

\section{P37-Abstract withdrawn}

\section{P38}

Azathioprine protects against poor bone health in mice with DSS-induced inflammatory bowel disease

\section{Stephanie Morgan, ${ }^{1}$ Kirsty Hooper, ${ }^{1}$ Katherine Halewood, ${ }^{1}$ Elspeth Milne, ${ }^{2}$ Colin Farquharson, ${ }^{2}$ Craig Stevens, ${ }^{1}$ and Katherine Staines ${ }^{1}$}

\author{
${ }^{1}$ School of Applied Sciences, Edinburgh Napier University, \\ Edinburgh, UK \\ ${ }^{2}$ Roslin Institute and R(D)SVS, University of Edinburgh, Edinburgh, UK
}

Individuals with inflammatory bowel disease (IBD) often present with poor bone health. The development of targeted therapies for this bone loss requires a better understanding of the underlying cellular mechanisms. Azathioprine is a commonly used drug for IBD management; however, its mechanisms of action, in particular its effects on the skeleton, are not yet fully understood. Here, we have utilized the dextran sulphate sodium (DSS) model of colitis in mice to examine the effects of Azathioprine on bone health. Ten-week-old male wild-type mice ( $n=6 /$ group) received $3.0 \%$ DSS in their drinking water for 4 days, following which they received tap water for a 14-day recovery period. Mice were treated by oral gavage daily throughout the experiment with clinically relevant doses of Azathioprine or vehicle control. Micro-computed tomography assessment of vehicle-treated DSS mice revealed a worsened trabecular architecture compared with vehicle-treated control mice. Specifically, DSS-treated mice exhibited decreased BV/TV (32\%), trabecular number (12\%), and trabecular thickness (12\%). In comparison, Azathioprine-treated mice were partially protected from the adverse effects of DSS, with no decreases in trabecular parameters observed. Histopathological analysis of the colon revealed no differences in the severity or extent of inflammation in Azathioprine-treated mice, in comparison to vehicle-treated mice, suggesting that Azathioprine has a direct protective effect against bone loss typical of IBD. Ongoing in vitro studies will determine the mechanism of action, and this will impact on our understanding of bone-protective agents given to patients with IBD. 
P39

Quality of bone formed by osteogenic stem/progenitor cell cultures

\section{Gurjit S Mandair, ${ }^{1}$ Pieter Steenhuis, ${ }^{1}$ Michael A Ignelzi Jr, ${ }^{1}$ and Michael D Morris ${ }^{2}$}

${ }^{1}$ School of Dentistry, University of Michigan, Ann Arbor, MI, USA

${ }^{2}$ Department of Chemistry, University of Michigan, Ann Arbor, MI, USA

The use of autologous stem/progenitor cells represents a promising approach to the repair of craniofacial bone defects. The calvarium is recognized as a viable source of stem/ progenitor cells that can be transplanted in vitro to form bone. However, it is unclear if bone formed in culture is similar in quality to that found in native bone.

Objective: In this time-dependent study, the quality of bone formed in osteogenic cultures were compared against calvarial bone from postnatal mice.

Methods: Stem/progenitor/stem cell fractions isolated from fetal mouse calvaria were plated onto fused-quartz slides. At confluence, the cells were cultured under osteogenic differentiation conditions for 28 days. At specific time intervals, the slides were removed, fixed, and examined by Raman microscopy ( $n=3$ slides for each time interval: 1, 4, 8, 14, 17, 25, and 28 days). Raman spectroscopic measures of cell nucleic acid/protein ratio, collagen/matrix ratio, mineral/matrix ratio, mineral crystallinity, collagen crosslinks ratio, and collagen disorder/order ratio were calculated. Similar measurements were also obtained from calvarial bone from 1-day-old postnatal mice $(n=3)$. After Raman microscopy, the slides were stained for mineral by Alizarin red and von Kossa staining techniques.

Results: The early decline in cell nucleic acid/protein ratios found with culture time was mirrored by a reciprocal increase in collagen/matrix ratios. The latter result indicated that the decline in cell proliferation preceded collagen accumulation. Mineral deposition was first detected at day 8 and was confirmed by Raman microscopy and mineral staining techniques. A bimodal pattern of mineralization was found with maxima mineral/matrix ratios at days 8 and 28. Collagen crosslinks ratios plateaued with culture time, whereas collagen order increased with culture time. Bone formed in culture at day 28 resembled native calvarial bone with comparable mineral/matrix ratios, collagen/matrix ratios, and collagen crosslinks ratios. In contrast, bone formed at day 28 was less ordered and less crystalline compared with calvarial bone.

Conclusion: Bone formed in osteogenic cultures exhibited the hallmarks of progressive bone matrix maturation and mineralization but could not fully replicate the high degree of collagen fibril order found in native bone. We acknowledge NIH grants R01 AR047969 and DE11530.

\section{P40}

Effects of in vitro glycation on bone collagen structure and order

Gurjit S Mandair, ${ }^{1}$ Matthew Karabetsos, ${ }^{1}$ Gloria G Vanrenterghem, ${ }^{1}$ Ramamoorthy Ayyalusamy, ${ }^{2}$ Michael D Morris, $^{2}$ and David H Kohn ${ }^{1}$

${ }^{1}$ School of Dentistry, University of Michigan, Ann Arbor, MI, USA
${ }^{2}$ Department of Chemistry, University of Michigan, Ann Arbor, MI, USA

Advanced glycation end products (AGEs) increase with age in bone but are exacerbated in patients with diabetes. Given that AGE formation in normal bone is slow, in vitro glycation of bone by high glucose or ribose concentrations can yield similar AGE levels that would otherwise take decades to reach in vivo. However, it is unclear if these glycating agents differentially modify bone toward increased collagen crosslinks, decreased collagen order, or both.

Objective: This in vitro study sought to clarify if bone collagen structure was differentially modified after glycation with high glucose or high ribose concentrations.

Methods: Powdered bovine bone samples were assigned to one of the three 3-week incubation groups ( $n=6$ per group): control, $0.6 \mathrm{M}$ glucose, or $0.6 \mathrm{M}$ ribose. Reactions were performed at $37^{\circ} \mathrm{C}$ in calcium-buffered Hank's solutions and $\mathrm{pH}$ was adjusted daily to 7.2. After 3 weeks, the control and glycated bone samples were dialyzed and then analyzed by Raman spectroscopy. Bonequality measures of collagen crosslinks ratios $\left(1657 / 1687 \mathrm{~cm}^{-1}\right)$ and collagen disorder/order ratios $\left(1248 / 1268 \mathrm{~cm}^{-1}\right)$ were calculated. Statistical differences between groups were determined by Student $t$ tests $\left({ }^{*} p<0.05\right)$.

Results: Bone incubated with ribose was colored pale yellow because of the presence of AGEs, whereas bone incubated with glucose was indistinguishable from control bone. Bone incubated with ribose had significantly increased collagen disorder/order ratios compared with control bone $(1.03 \pm 0.02$ versus $0.97 \pm 0.03$, respectively). No significant differences between bone glycated with glucose and control bone were found using either collagen crosslinks ratios $(4.47 \pm 0.54$ versus $4.51 \pm 0.33$, respectively) or collagen disorder/order ratios $(0.99 \pm 0.03$ versus $0.97 \pm 0.03$, respectively). The significant increase in collagen disorder ratios found in bone glycated with ribose indicated that the intermolecular distances between collagen chains may have increased with the loss of long-range fibril order. The lack of significant glucose-induced changes to collagen structure may reflect its slower reactivity compared with ribose.

Conclusion: This in vitro glycation study demonstrated the detrimental effects of ribose on bone collagen order, which may aid in the understanding of fragility fractures reported in patients with diabetes. We acknowledge NIH grants R01 AR05210 and U01 DE023771.

\section{P41}

A study of the relationships between fracture risk and ethnicity

\author{
Premila Kadamban, ${ }^{1}$ and James Galloway ${ }^{2}$ \\ ${ }^{1}$ Rheumatology, Basildon University Hospital, Basildon, UK \\ ${ }^{2}$ Rheumatology, King's College, London, UK
}

Background: Osteoporosis is a major worldwide public health issue with steeply rising prevalence in a world with a rapidly aging population. Fractures can be detrimental, significantly affecting an individual's quality of life or it can be fatal at times. Fracture risk assessment and taking necessary measures to prevent fractures are paramount.

Aims: The objective of this study is to assess the effect of ethnicity on fracture risk. 
Methods: This study is done utilizing routinely collected data of 7428 patients who had bone densitometry from July 2008 to November 2015. Bone density measurements were obtained using GE Prodigy scanner from the spine and femoral neck. Ethnicity data were self-reported. Incident fracture data were also available through linkage of the patient unique identifier to the Hospital Episode Statistics submission data for the trust. This enabled capturing of coded fracture events for which the patients sought care within the base hospital.

Results and Discussions: The prevalence of osteoporosis and fracture risk is considerably lower in people of Afro-Caribbean origin compared with that of whites. The odds ratio of osteoporosis in Afro-Caribbeans is 0.43 and the hazard ratio (HR) of age-, sex-, BMI-, and bone mass density (BMD)-adjusted fracture risk is 0.5 . The prevalence of osteoporosis is similar in whites and Asians; however, the fracture risk is considerably lower $(\mathrm{HR}=0.56)$ in the latter group. Certain factors that change with ethnicity play an important role in determining fracture risk. Conclusion and Recommendations: We conclude that the risk of osteoporosis and fracture risk is significantly lower in people of Afro-Caribbean origin compared with whites. Although the risk of osteoporosis is similar in whites and Asians, the fracture rate is considerably lower in Asians. Thus, ethnicity has a major impact on fracture risk. As ethnicity has a significant impact on fracture risk, it has got to be considered in assessing fracture risk. As bone density only partially explains the fracture risk, we recommend further studies to analyze the other factors that determine or contribute to the fracture risk.

\section{P42}

Using ante-mortem consented bone samples as agematched controls in hip fracture research

\section{Linda Skingle, ${ }^{1}$ Timothy Vaughan-Lane, ${ }^{2}$ Maria Wright, ${ }^{2}$ Cecilia Brassett, ${ }^{2}$ and Kenneth Poole ${ }^{1}$}

${ }^{1}$ Department of Medicine, University of Cambridge, Cambridge, UK ${ }^{2}$ Human Anatomy Teaching Group, Department of Physiology, Development and Neuroscience, University of Cambridge, Cambridge, UK

Objectives: It is increasingly difficult to obtain control bone samples to compare with biopsies from our studies of patients with hip fracture. The main source of such bone is cadaveric material, but the MRC decision tree (https://tinyurl.com/ y9wsok42 Annex 3) made clear to us that Cambridge pre2006 archival specimens did not fulfill modern consent requirements. We set out to acquire ante mortem consented bone tissue that we could use within the Human Tissue Act 2004. Methods: Under the Human Tissue Act 2004, which came into force on September 1, 2006, potential donors must provide written and witnessed consent, before their death, for their bodies to be used for the purpose of anatomical examination after their death. After discussion with Designated Individual of the Human Anatomy Centre (HAC), Department of Physiology, Development and Neuroscience, University of Cambridge, we submitted a research proposal that was reviewed and accepted. After inspection and approval of the security and suitability of the storage arrangements of our bone lab, an agreement for transfer and loan of cadaveric material for bone research was signed. Donors that were suitable in terms of age bracket and cause of death were identified. After dissection and disarticulation, femoral head-neck specimens were transferred to our HTA post-mortem licensed lab for a specified period. An amendment to the FEMCO study (LREC07/H0305/61) permitted the samples to be studied. Samples were scanned in an HRpQCT scanner and bone morphometry was performed using ImageJ/boneJ. ${ }^{(1)}$

Results: Samples were well matched (case mean value, control mean value $\pm S D$ ) for age ( $82 \pm 5$ years versus $83 \pm 6$ ), weight $(62 \pm 17 \mathrm{~kg}$ versus $60 \pm 12)$, bone volume (BV/TV; $28.9 \pm 3.1 \%$ versus $29.8 \pm 2.8$, and femoral head diameter $(45.8 \pm 4.0 \mathrm{~mm}$ versus $46.8 \pm 4.6$ ), but participants differed in height $(161 \pm 10 \mathrm{~cm}$ versus $170 \pm 7 ; p=0.0062)$

Conclusion: Approaching and working with our local anatomy school provided us with fully ante mortem consented anonymized cadaveric samples that were used as controls for our study into differences in the microstructure of the femoral head in hip fracture.

\section{Reference}

1. Doube M, Klosowski MM, Arganda-Carreras I, et al. BoneJ: free and extensible bone image analysis in ImageJ. Bone. 2010;47 (6):1076-9.

\section{P43}

Reliable change index in the evaluation of joint space loss: a novel method for assessing osteoarthritis progression

\section{Camille Parsons, ${ }^{1}$ Andrew Judge, ${ }^{2}$ Kirsten Leyland, ${ }^{2}$ Hazel Inskip, ${ }^{1}$ and Cyrus Cooper ${ }^{1,3,4}$}

\author{
${ }^{1}$ MRC Lifecourse Epidemiology Unit, University of Southampton, \\ Southampton General Hospital, Southampton, UK \\ ${ }^{2}$ Musculoskeletal Research Unit, University of Bristol, Bristol, UK \\ ${ }^{3}$ NIHR Southampton Nutrition Biomedical Research Centre, \\ University of Southampton and University Hospital Southampton \\ NHS Foundation Trust, Southampton, UK \\ ${ }^{4}$ NIHR Oxford Biomedical Research Centre, University of Oxford, \\ Oxford, UK
}

Objective: We aimed to assess the effectiveness of the reliable change index $(\mathrm{RCl})$ as a novel approach to estimating osteoarthritis (OA) progression using longitudinal knee joint space width (JSW) measurements, while accounting for the presence of measurement error.

Methods: Data from 3469 participants of the Osteoarthritis Initiative (OAI), an American multi-center, longitudinal study, were obtained. Knee JSW measurements were taken from radiographic images at baseline and at $12,24,36,48,72$, and 96 months. The $\mathrm{RCl}$ allows for identification of whether observed change is statistically reliable in the presence of measurement error. An $\mathrm{RCl}$ score was calculated for each participant, using the version developed by Christensen and Mendoza.

Results: Between consecutive study visits, 53\% to $64 \%$ of participants had a crude decrease in JSW between study visits. Implementation of the $\mathrm{RCl}$ indicated that considerably fewer participants had a statistically reliable decrease in JSW, eg, 3.1\% participants were identified between baseline and 12-month study visits compared with 53\% crude decrease. Similar proportions of statistically reliable decreases were observed between all other OAI study visits. Around $40 \%$ of participants had crude increase in JSW between the pairs of study visits under consideration. Use of the $\mathrm{RCl}$ dramatically reduced the number of participants that were identified as having an increase, eg, between 72 - and 96-month study visits, only $1.4 \%$ 
were identified compared with 39\% that were identified using crude differences. As increases in JSW are biologically extremely rare, this shows the impact of measurement error if crude differences are assessed without accounting for measurement error. Conclusion: The $\mathrm{RCl}$ provides a useful method to identify change in JSW, removing many of the apparent changes that are likely to be due to measurement error. This method appears to be useful for assessing change in JSW clinical and research settings from radiographs.

\section{P44}

Bone formation and vascular calcification are differentially affected by $\mathrm{N}$-acetylcysteine

\section{Lucie E Bourne, ${ }^{1}$ Jessal J Patel,, ${ }^{1,2}$ Ellen Neven, ${ }^{3}$ Patrick D'Haese, ${ }^{3}$ Caroline Wheeler-Jones, ${ }^{1}$ and Isabel R Orriss ${ }^{1}$}

\author{
${ }^{1}$ Department of Comparative Biomedical Sciences, Royal \\ Veterinary College, London, UK \\ ${ }^{2}$ School of Life and Medical Sciences, University of Hertfordshire, \\ Hatfield, UK \\ ${ }^{3}$ Laboratory of Pathophysiology, Department of Biomedical \\ Sciences, University of Antwerp, Antwerp, Belgium
}

Vascular calcification (VC) involves hydroxyapatite deposition in the arteries and is a significant risk factor for future cardiovascular events. Historically believed to share similarities with bone mineralization, emerging evidence suggests that there are several key differences between these two processes. For example, antioxidants appear to inhibit VC but promote bone formation. $\mathrm{N}$-acetylcysteine (NAC), the acetylated form of $\mathrm{L}$-cysteine, displays direct and indirect antioxidant properties. This study investigated the effects of NAC on VC and bone mineralization in vitro and in vivo. Human umbilical arteryderived vascular smooth muscle cells (VSMCs) and mouse osteoblasts were cultured in mineralizing medium (1 mM $\beta$ glycerophosphate, $1 \mathrm{mM}$ sodium phosphate, $50 \mu \mathrm{g} / \mathrm{mL}$ ascorbate) and treated with NAC (0.5-5 mM) for up to 7 and 21 days, respectively. The effect of NAC on cell function, survival, and gene expression was investigated using established assays. NAC potently increased in vitro bone formation ( $\leq 10$-fold, $p<$ 0.001 ) but reduced VSMC calcification $(\leq 80 \%, p<0.001)$. NAC increased osteoblast TNAP activity, soluble collagen levels, and gene expression (eg, osteocalcin, osteopontin) up to threefold $(p<0.001)$. Cell number and viability were unaffected. Conversely, NAC had no effect on VSMC TNAP activity but reduced VSMC apoptosis by up to $40 \%$ ( $p<0.001$ ). VC is associated with decreased expression of VSMC-associated genes (eg, $\alpha$-SMA, SMemb, SM22 $\alpha$ ). NAC prevented this reduction in calcifying VSMCs by up to threefold. NAC was administered daily $(300 \mathrm{mg} / \mathrm{Kg}$ ) to Wistar rats fed a warfarin/ vitamin K1-enriched diet for 10 weeks $(n=9-10)$. VC and trabecular/cortical bone parameters were assessed using $\mu \mathrm{CT}$. Treatment with NAC decreased aortic calcification by up to $25 \%(p<0.05)$. Trabecular and cortical bone parameters were unaffected. However, a negative correlation between the level of aortic calcification and trabecular bone volume $(p=0.0237)$ was observed. Taken together, our findings suggest that NAC exerts opposing effects on VC and bone mineralization and could represent a potential therapy for VC that does not negatively affect the skeleton. Current work is investigating the mechanistic basis for these effects.
Age at initiation of antiretroviral therapy predicts low bone density in Zimbabwean children with vertically acquired HIV infection

\section{April Hartley, ${ }^{1,2}$ Ruramayi Rukuni, ${ }^{3,4}$ Nicola Crabtree, ${ }^{5}$ Cynthia Mukwasi, ${ }^{6}$ Edith Majonga, ${ }^{3,4}$ Grace McHugh, $^{3}$ Hilda Mujuru, ${ }^{7}$ Rashida Ferrand, ${ }^{3,4}$ and Celia L Gregson ${ }^{1}$}

\author{
${ }^{7}$ Musculoskeletal Research Unit, University of Bristol, Bristol, UK \\ ${ }^{2}$ Integrative Epidemiology Unit, University of Bristol, Bristol, UK \\ ${ }^{3}$ Biomedical Research and Training Institute, Harare, Zimbabwe \\ ${ }^{4}$ Clinical Research Department, Faculty of Infectious and Tropical \\ Diseases, London School of Hygiene and Tropical Medicine, \\ London, UK \\ ${ }^{5}$ Department of Endocrinology and Diabetes, Birmingham \\ Children's Hospital, Birmingham, UK \\ ${ }^{6}$ Department of Radiology, College of Health Sciences, University of \\ Zimbabwe, Harare, Zimbabwe \\ ${ }^{7}$ Department of Paediatrics and Child Health, University of \\ Zimbabwe, Harare, Zimbabwe
}

Objectives: Global roll-out of antiretroviral therapy (ART) means more children with vertically acquired HIV infection are surviving into adolescence; however, impacts on bone and muscle development are unclear. We aimed to understand the epidemiology of bone density, muscle mass, and function in HIV-infected (HIV+) and uninfected (HIV-) Sub-Saharan African children.

Methods: We performed cross-sectional analyses in a population of $\mathrm{HIV}+$ children receiving ART for $\geq 6$ months and HIV- children attending primary health clinics in the same region of Zimbabwe (aged 6 to 16 years). Bone density was assessed by DXA and total-body less-head bone mineral content for lean mass adjusted for height (TBLH-BMC ${ }^{\mathrm{LBM}}$ ) and $\mathrm{L}_{1}$ to $\mathrm{L}_{4}$ lumbar spine bone mineral apparent density (LS-BMAD) Z-scores were derived. Differences in TBLH$\mathrm{BMC}^{\mathrm{LBM}} Z$-score, LS-BMAD Z-score, muscle mass, and strength were assessed by multivariable linear regression, adjusting for age, sex, and puberty (and height except for TBLH-BMC ${ }^{\text {LBM }}$ Z-score).

Results: $97 \mathrm{HIV}+$ children (mean age 12.7 [SD 2.5] years, 53\% female) and 77 HIV- children (10.0 [2.9] years, 52\% female) were assessed. HIV+ children were older and hence taller $(141.5 \mathrm{~cm}$ [11.4] versus $134.0 \mathrm{~cm}$ [16.6]); however, stunted growth (height for age $Z$-score $<-2$ ) was more prevalent in HIV+ children (32\% vs $5 \% ; p<0.01)$. Low TBLH-BMC ${ }^{\text {LBM }}$ and LS-BMAD (Z-score $<-2$ ) were also more common in HIV + children (13\% versus $3 \%$ and $15 \%$ versus $1 \%$, respectively, both $p<0.01$ ). In adjusted analyses, LS-BMAD Z-score was 0.33 SD lower $(95 \% \mathrm{Cl}-0.01$, $0.67)$ in $\mathrm{HIV}+$ children $(p=0.055)$, but no differences were observed in TBLH-BMC ${ }^{\mathrm{LBM}}$, lean mass, or grip strength. Age at ART initiation was inversely associated with both TBLH-BMC ${ }^{\text {LBM }}$ and LS-BMAD Z-scores, with a 1-year delay associated with a 0.08 SD decrease in TBLH-BMC $C^{\mathrm{LBM}}(p=0.027)$ and a 0.13 SD decrease in LS-BMAD $(p<0.01)$. Tenofovir use was associated with substantially lower LS-BMAD.

Conclusion: Vertically acquired HIV infection is associated with stunting and, after accounting for body size, low LS-BMAD, suggesting HIV infection detrimentally affects trabecular bone development. Delays in initiating ART appear to significantly impact bone density. Findings demonstrate the detrimental 
impact of delayed diagnosis and highlight the need for immediate ART initiation.

\section{P46}

Inspecting multiscale damage in human bone using an X-ray image algorithm

\section{Jiye Chen ${ }^{1}$ and Jin Luo ${ }^{2}$}

${ }^{1}$ Faculty of Technology, University of Portsmouth, Portsmouth, UK ${ }^{2}$ School of Applied Sciences, London South Bank University, London, UK

Human bone is subjected to damage due to aging and disease. In clinical practice, it is currently challenging to inspect bone damage using traditional $\mathrm{X}$-ray technologies such as quantitative computed tomography (QCT) due to their low resolutions. Recent advances in X-ray imaging techniques have enabled the development of high-resolution peripheral quantitative computed tomography (HR pQCT) with image resolution up to 40 micrometers. It is thus possible that these images may be used to detect bone micro damages for the non-invasive assessment of skeletal status clinically. To realize this possibility, an X-ray image correlation algorithm (XRICA) was developed recently in the University of Portsmouth for inspecting damage state in bone. The XRICA has the capability to automatically identify the surface and internal damages in bone at multiscale level. This study applied the novel XRICA technology to detect damages from micro-CT images of human trabecular bone samples. In Figure 1, the first and second diagrams from left are selected micro-CT images scanned along the length of a cylinder bone sample (diameter $6.3 \mathrm{~mm}$, length $25 \mathrm{~mm}$ ), which had been compressively loaded to failure. The resolution for the scanning was set at 1.5 micrometers. The third and fourth diagrams in Figure 1 show the XRICA identified damages. The third one was used as a reference image. The fourth one was identified with several damages presented by red colors, which indicate the location, area, and level of each damage. Figure 2 shows a scanned image of a vertebral bone sample using a resolution of 70 micrometers together with the XRICA identified damages. This investigation shows that the XRICA is able to detect bone damage on micro-CT images with resolutions ranging from 1.5 to 70 micrometers. It can potentially provide a new approach to enhance clinical assessment of bone status in the future.

\section{P47}

Harnessing clay nanoparticles to stabilize and enhance bioactive extracellular matrix for bone regeneration application

\section{Vikash H Dodhia, Gianluca Cidonio, Yang-Hee Kim, Richard OC Oreffo, and Jonathan I Dawson}

Bone and Joint Research Group, Centre for Human Development, Stem Cell and Regeneration, Faculty of Medicine, University of Southampton, Southampton, UK

Introduction: Biomaterials can provide support, aid as a scaffold for bone regeneration, and serve as delivery vehicles for biomolecules for bone reparation. Hydrogels synthesized from bone extracellular matrix (ECM), obtained from decellularized and demineralized bovine bone tissue, contain endogenous biomolecules $^{(1)}$ and have been shown to promote bone regeneration. We have recently developed human-derived bone ECM hydrogels; however, hECM hydrogels are mechanically weak and rapidly degrade. The nanoclay, Laponite, is able to localize growth factors, enhance osteogenesis, and improve the mechanical properties of hydrogels. ${ }^{(2,3)}$ The aim of the current study was to explore the potential of Laponite to improve the mechanical stability and bioactivity of hECM for bone regeneration strategies.

Methods: Laponite $\left(28 \mathrm{mg} \mathrm{m}^{-1}\right), \mathrm{hECM}\left(20 \mathrm{mg} \mathrm{ml}^{-1}\right)$, and varying concentrations of sodium polyacrylate (ASAP) (MW:15KDa) were mixed before gelation at $37^{\circ} \mathrm{C}$. ASAP was employed as a peptizer ${ }^{(4)}$ to improve dispersion of Laponite in ECM. The gelation, rheological, and optical properties of the gels were assessed. Human bone marrow stromal cell (HBMSC) viability in response to the composite hydrogels was also determined.

Results: Amplitude sweep measurements were carried out with a shear strain linear increase from 0 to $100 \%$, revealing that the storage modulus of the hECM-Laponite composite gel was higher than the hECM gel alone $(340.1 \pm 79.3$ and $1.68 \pm 0.77 \mathrm{~Pa}$, respectively). The addition of ASAP (0.06-0.24\%) produced a weakened gel with a reduced storage modulus. However, addition of ASAP produced a visibly more homogenous gel, as determined by turbidimetric assay (absorbance of $0.263 \pm 0.005$ and $0.138 \pm 0.008 \mathrm{AU}$ without ASAP and $0.12 \%$ ASAP, respectively). HBMSCs cultured on hECM-Laponite films were viable after 7 days, showing that these gel composites with increasing concentrations of ASAP provide an environment for cell infiltration and proliferation.

Conclusion: The current studies have demonstrated the addition of Laponite enhances mechanical properties and stability of hECM, confirming its promise as a bioactive scaffold to augment bone regeneration.

\section{References}

1. Sawkins MJ, Bowen W, Dhadda P, et al. Hydrogels derived from demineralized and decellularized bone extracellular matrix. Acta Biomater. 2013;9(8):7865-73.

2. Dawson Jl, Kanczler JM, Yang XB, Attard GS, Oreffo RO. Adv Mater. 2011;23(29):3304-8.

3. Gibbs DM, Black CR, Hulsart-Billstrom G, et al. Bone induction at physiological doses of BMP through localization by clay nanoparticle gels. Biomaterials. 2016;99:16-23.

4. Wang Q, Mynar JL, Yoshida M, et al. High-water-content mouldable hydrogels by mixing clay and a dendritic molecular binder. Nature. 2010;463(7279):339.

\section{P48}

Stimulation of bone cell function using osteogenic factorloaded poly-lactic-co-glycolic (PLGA) nanoparticles in lightcurable scaffolds

\section{Michael Glinka, ${ }^{1}$ Gianluca Cidonio, ${ }^{1}$ Jin Geng, ${ }^{2}$ Ewa Czekańska, ${ }^{1}$ Yang-Hee Kim, ${ }^{1}$ Jonathan I Dawson, ${ }^{1}$ Shoufeng Yang, ${ }^{1}$ Khoon Lim, ${ }^{3}$ Tim Woodfield, ${ }^{3}$ Mark Bradley, ${ }^{2}$ and Richard Oreffo ${ }^{1}$}

${ }^{1}$ Faculty of Medicine, University of Southampton, Southampton, UK ${ }^{2}$ School of Chemistry, University of Edinburgh, Edinburgh, UK

${ }^{3}$ Department of Orthopaedics Surgery and Musculoskeletal Medicine, University of Otago Christchurch, Christchurch, New Zealand 
Introduction: Tissue engineering offers cost-effective solutions and efficacious approaches to aid tissue repair with the application of osteogenic factor-loaded hydrogels that support differentiation of human stem cells. However, precise spatiotemporal control and delivery of osteogenic factors at physiological concentrations remain a significant challenge.

Objectives: The development of a strategy to deliver osteogenic factors such as vitamin $C$ and vitamin $D_{3}$ by encapsulation in PLGA nanoparticles (NPs) for efficient release and stimulation of bone marrow stem cells (HBMSCs) within three-dimensional (3D) printed gelatin methacryloyl (GelMA) hydrogel scaffolds.

Methods: The characterization of the mechanical properties of GelMA was examined using amplitude and frequency sweep tests at increasing shear strain and frequency, respectively. Gelation analysis was completed at $1 \%$ shear and $1 \mathrm{~Hz}$. Cell viability and proliferation were analyzed by seeding HBMSCs on the surface and staining with calcein-AM and Vybrant DiD and quantified with ImageJ software. Nanoparticles were analyzed with reverse phase high-performance liquid chromatography (HPLC) by eluting with $\mathrm{H}_{2} \mathrm{O}$ and methanol for vitamin $\mathrm{C}$ and vitamin $D_{3}$, respectively. All tests were performed at $37^{\circ} \mathrm{C}$.

Results: Rheological analysis of $10 \%(\mathrm{w} / \mathrm{v})$ GelMA bioink demonstrated the shear-thinning properties before crosslinking and rapid gelation via visible light $(450 \mathrm{~nm})$ to generate a stable gel. Amplitude sweep test of GelMA hydrogels showed a static gel with a storage modulus of $1.0 \mathrm{kPa}$ capable of withstanding over $6 \mathrm{kPa}$ shear deformation. SEM analysis of hydrogel porosity showed an average pore size of $210 \pm 90 \mu \mathrm{m}$ and cell proliferation analysis showed a $>20$-fold increase $(p<0.05)$ in HBMSC cell numbers after 28 days after initial seeding of $1 \times 10^{5}$ HBMSCs on the GelMA. HPLC analysis of the PLGA NPs confirmed encapsulation of $80 \mu \mathrm{g} / \mathrm{mg}$ and $0.45 \mu \mathrm{g} / \mathrm{mg}$ of vitamin C and vitamin D3, respectively. The current work is centered on the release of vitamin $C$ and vitamin $D_{3}$ on HBMCSs. Conclusion: The ability to incorporate PLGA NPs in GeIMA bioink provides a promising approach to the controlled release of bioactive factors to promote bone repair.

\section{P49}

Methods for determination of carbonate position in hydroxyapatite lattice

\section{Emily Arnold, Charlene Greenwood, and Keith Rogers}

Cranfield Forensic Institute, Cranfield University, Shrivenham, UK

Introduction: The amount of carbonate present within the hydroxyapatite (HA) lattice has been reported to differ between osteoporotic (OP) and non-diseased bone. ${ }^{(1)}$ Carbonate occupies two sites within the HA lattice: phosphate (B-type) and ion channel (A-type) sites. Studies employ infrared spectroscopy (IR) to quantify A- and B-type carbonate. Carbonate substitution increases dissolution rates, potentially explaining increased bone loss in osteoporosis. Use of the $\mathrm{v}_{2} \mathrm{CO}_{3}{ }^{2-}$ absorption band in carbonated HA is accomplished by deconvolution of spectra; this is controversial as there is overlap between A- and B-type $\mathrm{v}_{2} \mathrm{CO}_{3}{ }^{2-}$ peaks and the $\mathrm{v}_{1} \mathrm{v}_{3} \mathrm{PO}_{4}{ }^{3-}$ peak. Other than IR, it has not been independently verified that carbonate substitutes into $A$ and $B$ sites in biological HA.

Pair distribution function (PDF) studies present a potential corroborative approach to provide distribution of carbonate within HA. Marisa and colleagues ${ }^{(2)}$ demonstrated that current
PDF techniques identify variations in the location of B-type carbonate in synthetic HAs. PDFs may be used to quantify carbonate in biological HA, but first studies of A- and B-type are needed to see if differences are present.

Methods: To identify differences stoichiometric, A-type, B-type, and combinations of A- and B-type HAs were simulated with varied $Q_{\max }$ values. Simulated PDFs were compared by creating difference plots. Goodness-of-fit parameters were observed for each $Q_{\max }$ value.

Results: Novel PDFs of A- and B-type HAs have been observed. Differences between A- and B-type can be seen due to different atomic distributions, mostly $r<10 \AA^{-1}$. Differences are seen between carbonated, stoichiometric HAs, and A- and B-type carbonated HAs, demonstrating that PDF analysis may be a useful tool for this research.

Conclusion: The differences observed between A- and B-type simulated carbonated HAs proposes that PDF studies may be used to corroborate other techniques with the potential to inform lattice chemistry and possible biomarkers for early detection of OP. Differences at low $r$ values necessitate data collection at higher $Q_{\max }$ values than are available using laboratory equipment. Further work is scheduled at Diamond Light Source on a range of synthetic and biological HAs.

\section{References}

1. Greenwood C, Clement JG, Dicken A, et al. Towards new material biomarkers for fracture risk. Bone. 2016;93:55-63.

2. Marisa ME, Zhou S, Melot BC, Peaslee GF, Neilson JR. Paracrystalline disorder from phosphate ion orientation and substitution in synthetic bone mineral. Inorg Chem. 2016;55:12290-8.

\section{P50}

Getting to the bare bones of the age-related changes to the mechanical and structural properties of the clavicle

\section{Hannah McGivern, Charlene Greenwood, Nicholas Marquez- Grant, and Peter Zioupos}

\section{Cranfield Forensic Institute, Cranfield University, Shrivenham, UK}

Fractures of the clavicle occur universally across the age spectrum, with mechanisms of injury ranging from high-impact automotive collisions to assault, sporting injuries, and accidental falls that frequently befall the elderly. As a result, clinical treatment is continuously required for what is widely considered to be one of the most frequently broken bones. ${ }^{(1,2)}$ Furthermore, with the recent surge in operative treatment of such injuries, ${ }^{(2)}$ it has become all the more important to understand the complex system of age-related changes to the mechanical and structural properties of the clavicle. The objective of this phase of our interdisciplinary investigation was to study and quantify changes to the mechanical competence and micro-architectural properties of clavicular trabeculae, and subsequently cortical bone, using micro-computed tomography.

The sample comprised 25 non-fractured, right clavicles from 19 male and 6 female individuals, with ages ranging from 12 to 59 years. A method of selecting the maximum volume of trabeculae in the medial epiphysis was developed, and VGStudio Max 2.1 was used to measure bone surface (BS/BV), bone volume fraction (BV/TV), and mean trabecular number (Tb.N), thickness (Tb.Th), and spacing (Tb.Sp). In addition to the use of this progressive technique of assessing bone quality, as well as 
quantity, a QRM MicroCT-HA calibration phantom was scanned under the same conditions to calculate volumetric tissue mineral density ( $\mathrm{V}$ TMD).

Our investigation has thus far indicated a statistically significant $(p<0.05)$ decrease in BV/TV and Tb.N with age and a statistically significant $(p<0.05)$ increase in Tb.Sp with age. While positive and negative correlations were observed between age and BS/ BV and Tb.Th, respectively, these relationships have not shown to be statistically significant. Bone mineral density (BMD) was subsequently calculated using vTMD, and both showed a decrease with age. Moreover, these findings will be correlated with additional physicochemical and mechanical techniques to develop a more comprehensive understanding of the agerelated changes that occur within the different hierarchical levels of the clavicle bone structure.

This doctoral research is funded by the EPSRC.

\section{References}

1. Kihlström C, Moller M, Lonn K, Wolf O. Clavicle fractures: epidemiology, classification and treatment of 2422 fractures in the Swedish Fracture Register; an observational study. BMC Musculoskelet Disord. 2017;18(1):82.

2. Micev AJ, Hsu D, Edwards SL, Marra G, Saltzman MD. The rising incidence of operative fixation of acute mid-shaft clavicle fractures. J Shoulder Elbow Surg. 2014;23(9):e237.

\section{P51}

NaQuinate treatment increases bone strength in ovariectomized rats

\author{
Stephanie Gohin, ${ }^{1}$ Robin Soper, ${ }^{2}$ Behzad Javaheri, ${ }^{1}$ Lars \\ Marius Ytrebø, ${ }^{3}$ Mark Hopkinson, ${ }^{1}$ Richard Meeson, ${ }^{4}$ David \\ Howat, ${ }^{2}$ Andrew Pitsillides, ${ }^{1}$ and Stephen Hodges ${ }^{1,2,3}$ \\ ${ }^{1}$ Department of Comparative Biomedical Sciences, Royal \\ Veterinary College, London, UK \\ ${ }^{2}$ Haoma Medica, Haoma Medica, London, UK \\ ${ }^{3}$ Anaesthesiology and Intensive Care Medicine, Tromsø University \\ Hospital, Tromsø, Norway \\ ${ }^{4}$ Institute of Orthopaedics and Musculoskeletal Science, University \\ College London, Stanmore, UK
}

Osteoporosis represents a major healthcare burden that is anticipated to increase. Emerging therapies are limited in efficacy and by their safety profile. NaQuinate, 2-methyl-1,4naphthoquinone with a branched-chain aliphatic hydrocarbon carboxylic acid at the 3 position has previously been demonstrated to preserve trabecular bone volume and architecture in mouse models of osteoporosis. Herein, we have expanded upon this original work to test whether NaQuinate also modifies bone mass and architecture in ovariectomized female rats. Female Sprague Dawley rats ( $n=10$ per group) were bilaterally ovariectomized and received either vehicle (OVX+Veh: $5 \%$ ethanol/PBS; i.p.) or a daily NaQuinate dose (OVX+NaQ: $250 \mu \mathrm{g} / \mathrm{Kg} / \mathrm{d}$; i.p.) for 6 weeks; an additional group of rats underwent sham surgery and received a vehicle. Trabecular and cortical architecture in the tibia was imaged with micro-CT ex vivo. Ovariectomy resulted in greater gain in body mass $(+25 \%, p<0.0001)$, which was unaffected by NaQuinate treatment. Ovariectomized rats showed the expected diminution in trabecular bone mass and structure (BV/TV: $-80 \%, p<0.0001$ ). Interestingly, NaQuinate treatment for 6 weeks improved trabecular bone volume $(+58 \%, p<0.05)$ compared with OVX+Veh, indicating a protection against the bone loss induced by ovariectomy. In the cortical compartment, NaQuinate treatment for 6 weeks increased cortical bone area, Imin, Imax, and the predicted tibial resistance to torsion (J) across a broad mid-diaphyseal region of the tibia compared with OVX+Veh. Direct measurements of bone strength will be determined to confirm these extrapolated data. To conclude, these results suggest that NaQuinate treatment effectively maintains bone integrity and improves bone strength in ovariectomized rats.

\section{P52}

Harnessing high-resolution virtual histology in three dimensions for understanding bone development in birds

\section{Katherine A Williams, ${ }^{1}$ Neil J Gostling, ${ }^{2}$ Gareth Dyke, ${ }^{3}$ Richard OC Oreffo, ${ }^{4}$ and Philipp Schneider ${ }^{1}$}

\author{
${ }^{1}$ Mechanical Engineering, Faculty of Engineering and the \\ Environment, University of Southampton, Southampton, UK \\ ${ }^{2}$ Biological Sciences, Faculty of Natural and Environmental \\ Sciences, University of Southampton, Southampton, UK \\ ${ }^{3}$ Department of Evolutionary Zoology and Human Biology, \\ University of Debrecen, Debrecen, Hungary \\ ${ }^{4}$ Bone and Joint Research Group, Faculty of Medicine, University of \\ Southampton, Southampton, UK
}

Objectives: Accurately estimating developmental age and life history traits in fossils is crucial for identifying and classifying extinct species and understanding how biological attributes evolved. The evolution of life history traits such as growth pattern is far from clear in birds, and development has been studied in only a handful of modern species. The exceptionally rapid growth of modern birds means aging methods based on annual incremental growth lines, used in other vertebrates, are inapplicable to birds and robust alternative methods remain to be established.

Analysis of avian intracortical bone microstructure, which varies both with age and tissue deposition rate, is a promising approach already used in palaeobiology. However, current thin section-based histological methods are destructive. Moreover, to date, most microstructural studies in avian bone are qualitative, 2D, and involve a limited range of extant species. The objective of this study was to investigate cortical bone microstructure and developmental age and life history traits in living birds and to identify phenotypes that can then be applied to examination of the fossil record, using minimally destructive high-resolution 3D imaging.

Methods: We imaged cortical bone from the midshaft of the femur, tibiotarsus, and humerus encompassing a complete growth series of modern domestic ducks (Anas platyrhynchos) using high-resolution synchrotron-based computed tomography (TOMCAT, SLS, $1.6 \mu \mathrm{m}$ voxel size, energy $21 \mathrm{keV}$, exposure $180 \mathrm{~ms}$ per projection over 1501 projections). Twenty-one naturally deceased individuals were scanned: a range of juveniles ( $<6$ months) and 3 adults ( 2 years).

Results: After segmentation of intracortical porosity by absolute thresholding, 2D and 3D visualization revealed cortical microstructural changes through development, including bone volume fraction, heterogeneity in apparent canal diameter across the cortex, and differences in canal orientation and 
delayed mineralization in the humerus compared with the leg bones. Variation between bones reflects limb use: In ducks, running occurs from hatching, while flight occurs later.

Conclusion: Next steps will be to quantify the observed variation in bone microstructure through the life course. These measures will be tested for correlation with developmental age to identify phenotypes that could be used to estimate age.

P53

'Stories from Bones' - the potential of 3D scanning and biomechanical modelling in the archaeological study of human skeletons

\author{
Stephanie Evelyn-Wright, ${ }^{1}$ Martin Browne, ${ }^{2}$ Christopher \\ Woods, ${ }^{2}$ Mark Mavrogordato, ${ }^{3}$ Kathryn Rankin, ${ }^{3}$ Oliver \\ Stocks, ${ }^{2}$ Alex Dickinson, ${ }^{2}$ and Sonia Zakrzewski ${ }^{1}$ \\ ${ }^{1}$ Archaeology Department, University of Southampton, \\ Southampton, UK \\ ${ }^{2}$ Bioengineering Science Research Group, University of \\ Southampton, Southampton, UK \\ ${ }^{3} \mu$-VIS X-Ray Imaging Centre, University of Southampton, \\ Southampton, UK
}

This presentation outlines the aims and methods used in the interdisciplinary 'Stories from Bones' project at the University of Southampton. We combine archeological investigation and biomechanical modelling to provide insight into ancient human skeletal material. The team, formed of members from the Archaeology and Bioengineering Science departments, has found a number of applications for surface and volume imaging technologies, which enhance the research data we collect and the stories that we tell. This presentation will briefly describe two cases from the Stories from Bones project, including details of the methods used and the outcomes.

Our first case describes analysis of a singular femur bone from a Christian cemetery in Kent, C.1250-1600. This pathological specimen from the University of Southampton's Osteoarchaeology department had a displaced spiral fracture in the mid-shaft, which partially healed with substantial malalignment. Finite element analysis performed from micro focus X-ray CT scans was used to predict the strain that would be generated by gait loads. Comparison to healthy physiological bone strain levels indicated the extent to which the subject would have been able to ambulate.

The second case study discusses a young adult, female skeleton from the Romano-British cemetery site of Alington Avenue, which exhibited a rare incidence of mesomelic dwarfism. Through the integration of clinical and palaeopathological data with archaeological and historical data, a biography was formed of the life of the individual within her specific Roman setting.

In both cases, surface scanning and 3D printing were used to create models to enhance public engagement events and to help improve accessibility of material to the public, particularly to children and individuals with visual impairments.

This brief introduction intends to demonstrate some of the interdisciplinary benefits that 3D scanning and modelling technologies can contribute to bone research, whether the specimens are an entire skeleton or just one element. This paper also highlights the potential of interdisciplinary research and public engagement impact.

\section{P54}

The feasibility of a unilateral high-impact exercise intervention to increase bone mineral density in postmenopausal women

\section{Chris Hartley, ${ }^{1}$ Jonathan Folland, ${ }^{1}$ Robert Kerslake, ${ }^{2}$ and Katherine Brooke-Wavell ${ }^{1}$}

${ }^{1}$ NCSEM, School of Sport, Exercise and Health Science, Loughborough University, Loughborough, UK

${ }^{2}$ Queen's Medical Centre, Nottingham University Hospitals, Nottingham, UK

Postmenopausal bone loss predisposes older women to increased risk of osteoporotic fracture. High-impact exercise has been shown to increase bone mineral density (BMD), although findings are mixed in postmenopausal women. Previous research using unilateral, high-impact (hopping) exercises demonstrated positive effects on femoral neck BMD in younger women ${ }^{(1)}$ and older men. ${ }^{(2)}$ The aim of this study was to assess whether postmenopausal women aged 55 to 70 years were capable of performing such exercise, and to determine whether the impact of the exercise would be of a sufficient magnitude to promote an osteogenic response.

Forty-two women (age $61 \pm 5$ years; BMI $23.9 \pm 3.5 \mathrm{~kg} / \mathrm{m}^{2}$ ) were recruited from the local community. After a warm-up, each participant was asked to perform a set of 10 consecutive hops on a force plate (9286AA, Kistler Instruments Ltd, London, UK). Hops were conducted on each leg with a period of rest between trials. The peak vertical ground reaction force (vGRF) for each hop was averaged to give a mean vGRF for each leg.

All but one of the participants could complete 10 consecutive hops, with the foot leaving the ground, on both legs. One participant required support to complete the hops. The mean vGRF, normalized for body weight (BW) was $2.1 \pm 0.2$ for the right leg and $2.2 \pm 0.2$ for the left leg.

An exercise intervention that has been shown to increase BMD was feasible in a group of postmenopausal women. The vGRFs generated during a series of hops are lower than seen in other studies using bilateral exercise ${ }^{(3)}$ but comparable to those seen using unilateral exercise in younger women (2.32 BW-2.59 BW) and older men (2.25 BW-2.72 BW). These findings indicate that it is feasible for postmenopausal women to carry out high-impact unilateral exercise and they were capable of generating impact forces of similar magnitude to those that increased BMD in other groups. Further research is needed to evaluate the effectiveness and safety of high-impact exercise in this population.

\section{References}

1. Bailey CA, Brooke-Wavell K. 2010.

2. Allison SJ, et al. 2013.

3. Cheng S, et al. 2002

\section{P55}

Enhancement of a sustained release of bone morphogenetic protein-2 from hyaluronan-bisphosphonate hydrogel by addition of laponite clay nanoparticles

\section{Yang-Hee Kim, ${ }^{1}$ Dmitri Ossipov, ${ }^{2}$ Richard OC Oreffo, ${ }^{1}$ and} Jonathan I Dawson ${ }^{1}$

${ }^{1}$ Bone and Joint Research Group, Institute of Developmental Sciences, University of Southampton, Southampton, UK 
${ }^{2}$ Science for Life Laboratory, Division of Polymer Chemistry, Uppsala University, Uppsala, Sweden

Introduction: In the field of bone tissue regeneration, hydrogels can play a crucial role in not only providing a micro-environment for cells but also by delivering bioactive molecules. A hyaluronan (HA) hydrogel with covalently linked bisphosphonate (BP) ligands (HABP) shows a sustained release of bone morphogenetic protein (BMP)-2 in vitro. As another hydrogel candidate as a molecule carrier, laponite (LAP) gel based on clay-nanoparticle was also shown to enhance BMP-2 localization in the gel, resulting in the improved bone formation.

Objectives: In this study, we focused on the effect of LAP in HABP (HABP-LAP) hydrogel on the in vitro and in vivo release profiles of BMP-2 for bone regeneration.

Methods: We prepared HABP, LAP, and HABP-LAP gels incorporating lysozyme, as a protein model of BMP-2, and incubated them in PBS for 7 days, after collecting supernatant 1 , 2 , and 6 hours and 1, 3, and 7 days after incubation. The concentration of lysozyme in the supernatant was measured. To evaluate in vivo release pattern, we prepared HABP, LAP, and HABP-LAP gels incorporating Cy7-labelled lysozyme and implanted them in the back subcutis of MF1 wild mice. At days $0,3,7,14,21$, and 28 , the mice were scanned by an in vivo imaging system to detect the intensity of the lysozyme in vivo. Results: In the in vitro release study, approximately 16 and 24 percentage of lysozyme were released from LAP and HABP gels, respectively, a day after incubation, while HABP-LAP gel released less than 2 percentage of lysozyme for the same time. In the in vivo release study, we could not detect any Cy7labelled lysozyme in HABP gel a day after implantation, while the lysozyme incorporated into LAP and HABP-LAP gels showed a strong Cy7 dye intensity even 28 days after implantation.

Conclusion: We demonstrated that HABP hydrogel by addition of LAP could enhance a sustained release of protein, which is crucial for bone tissue regeneration.

Acknowledgments: Supported by an EPSRC fellowship (grant number EP/L010259/1).

\section{P56}

Osseointegrated implants for lower limb amputees: evaluation of bone mineral density

\section{Seamus Thomson, William Lu, and Munjed Al Muderis}

Clinical Research, The Osseointegration Group of Australia, Sydney, Australia

Background and Aims: The use of dual-energy X-ray absorptiometry (DXA) is a standard clinical procedure for the evaluation of bone mineral density (BMD). Amputee patients are known to have decreased $B M D$ and an increased risk of osteoporosis in the affected proximal femur and hip region. The major cause of these issues in these patients is the absence of adequate loading, leading to bone resorption in accordance to Wolff's law. In this paper, we present a prospective study reporting changes in BMD among amputees who received osseointegrated implants to determine if the loading through the osseointegrated implant can overcome the bone resorption issues.

Methods: This is a prospective study of 33 patients, consisting of 24 males and 9 females, aged 22 to 77 (mean $=51.0 \pm 2.0$ ) years with 1- and 2-year follow-up. Selection criteria included age over 18 years and unilateral amputees with socket-related problems. All patients received osseointegrated implants press-fitted into the amputated limb. BMD was assessed using DXA in the femoral neck (operative and contralateral) and lumbar spine $\left(L_{2}\right.$ to $L_{4}$ ) regions, and corresponding $Z$-scores were generated. DXA scans were taken preoperatively as well as 1 year and 2 years after osseointegration surgery.

Results: Mean BMD and Z-scores of spine and operative and contralateral sides were generated for all patients. Dependent $t$ tests were used to test for significant differences $(p<0.05)$ preoperative, 1 year, and 2 years for mean changes in BMD and $Z$ scores after surgery. Analysis of the BMD and Z-scores indicated that patients showed improvements at 1 year post-surgery.

Conclusion: These results suggest that osseointegrated implants are effective at encouraging bone growth and restoring BMD levels for amputees within a short period of time postsurgery. Osseointegrated implants therefore have the potential to address stress distribution issues associated with socket prostheses and restore the normal bone-loading regime in lower limb amputees.

\section{P57}

Osseointegrated implants for trans-femoral amputees: radiographic evaluation of bone remodeling

\section{Seamus Thomson, William Lu, and Munjed Al Muderis}

Clinical Research, The Osseointegration Group of Australia, Sydney, Australia

Background and Aims: Osseointegration is a novel method to overcome persistent socket prosthetic issues in amputees by anchoring a transcutaneous implant directly onto the skeletal residuum. Although similar technologies have been widely applied in the area of hip and knee arthroplasty, little evidence exists in the literature reporting the bone remodeling effects of osseointegrated implants. Stress shielding results in the reduction of bone density due to the implant removing the stress that is usually exerted on the bone, which greatly reduces implant stability. This paper investigates the bone remodeling effect and quantifies it in two of the most common osseointegration implants.

Methods: This is a prospective study of 50 patients with transfemoral amputations, consisting of 35 males and 15 females, aged 20 to 73 (mean 48.2) years at surgery, with minimum 2-year follow-up. Two implants, the Integral Leg Prosthesis (ILP) and Osseointegrated Prosthetic Limb (OPL), with differences in tapering, coating, and bone ingrowth regions were examined. Radiographs were taken at 6 months and 1, 2, and 5 years postsurgery. The surrounding bone was defined using inverse Gruen zones and graded into 5 levels of bone growth or resorption.

Results: Results obtained at 1- and 2-year follow-ups were compared with the 6-month follow-up values as a baseline. Significant bone growth near the proximal zones of the implant was observed on patients with the ILP implant. This was accompanied by significant resorption toward the distal end indicating the occurrence of stress shielding. The OLP implant demonstrated much more uniform bone density throughout the length of the implant.

Conclusion: Overall, the patterns of bone remodeling after osseointegration showed similarities to those seen on hip stems 
with a press-fit design. Of the two osseointegration implants examined in this paper, the OLP implant exhibited less stress shielding effects and is expected to provide better long-term stability.

\section{P58}

Serum adiponectin concentrations are inversely associated with bone mineral density in a community-based cohort of middle-aged women

\section{Nigel Arden, ${ }^{1}$ James R. Edwards, ${ }^{1}$ Stefan Kluzek, ${ }^{1}$ Aneka Sowman, ${ }^{1}$ Maria T Sanchez-Santos, ${ }^{1}$ Deborah Hart, ${ }^{2}$ and Tim D Spector ${ }^{3}$}

${ }^{1}$ NDORMS, University of Oxford, Oxford, UK

${ }^{2}$ Department of Rheumatology, St Bartholomew's Hospital Medical College, London, UK

${ }^{3}$ Department of Twin Research and Genetic Epidemiology, King's College London, London, UK

Introduction: Osteoporosis (OP) is an increasing health concern globally. Despite identification of several risk factors associated with osteoporosis development, there remains a need to identify reliable biomarkers for the prediction of OP incidence. Epidemiological studies to date have provided conflicting results regarding the effect of circulating concentrations of the adipokine adiponectin on bone mineral density (BMD) and OP development. This study aimed to assess whether serum concentration of adiponectin is associated with BMD or change in BMD over 5 years in a cohort of middle-aged women.

Methods: We used data from the community-based Chingford Cohort Study. Serum adiponectin levels were measured in fasting blood samples between years 8 and 10 (ELISA, R\&D Systems, USA) and dual-energy X-ray absorptiometry (DXA) scan (QDR 1000, Hologic, USA) was performed to assess BMD for total hip and lumbar spine $\left(L_{1}\right.$ to $\left.L_{4}\right)$ at years 10 and 15 . BMD change was defined as the difference between the initial (year 10) and the last (year 15) BMD measures. Cross-sectional and longitudinal associations between serum adiponectin concentrations and BMD and change in BMD at the total hip and $L_{1}$ to $L_{4}$ spine were analyzed using linear regression models, adjusted for age.

Results: 776 women (mean age 62.5 years) were included for the cross-sectional study and 560 women (mean age 67 years) for the longitudinal study. The median (interquartile range) adiponectin concentration was $17,160(11,840-24,200) \mathrm{ng} / \mathrm{mL}$. We found an inverse association between serum adiponectin concentration and BMD at the hip and spine ( $p \leq 0.001)$, which was not significantly attenuated after adjusting for age. In contrast, levels of adiponectin at year 10 were not associated with any significant change in BMD in the same region over a 5-year follow-up.

Conclusion: Serum adiponectin levels are inversely associated with BMD but cannot predict future change of the BMD. Future study should seek to elucidate the effects of the several circulating adiponectin homo-oligomeric complexes of varying molecular weights and expression of the adiponectin receptors AdipoR1 and AdipoR2 on BMD in the middle-aged population.

\section{P59}

Urocortin: a novel inhibitor of the differentiation of human osteoclasts
Omar Ismail, Joshua Coxon, Paul Townsend, Rebecca Jones, and Kevin Lawrence

Division of Cancer Sciences, University of Manchester, Manchester UK

Osteoporosis is a debilitating and potentially fatal disease affecting 200 million people worldwide. An imbalance between osteoclasts and osteoblasts leads to micro-architectural defects and low bone mass, culminating in serious fragility fractures, such as femoral neck fractures. Treatments currently used are either not completely effective or have serious side effects when used long term; thus there is a need for more effective therapies. Urocortin1 (Ucn1), a relative of Corticotropin-releasing factor (CRF), is a small peptide involved in the stress response and has previously been shown to inhibit murine osteoclast activity. We hypothesize that Ucn1 will have a similar effect in human osteoclasts, and extend this further by also investigating the role of CRF and Ucn2 (a paralogue of Ucn1) and their downstream signalling pathways in human osteoclasts.

Human osteoclasts were cultured from isolated human PBMCs, obtained from the blood bank, in the presence of M-CSF, RANKL, and Ucn ligands to assess the effect on differentiation. The cultured cells were stained with DAPI and Phalloidin to assess nuclei and actin, respectively, and visualized under $20 \times$ magnification. The number of mature human osteoclasts per field were counted, where a mature osteoclast was a large actinrich cell with $>3$ nuclei.

A significant decrease was observed in the number of osteoclasts per field when treated with Ucn $1(17.80 \pm 3.02, p$ $<0.01)$ and Ucn2 $(22.00 \pm 5.47, p<0.05)$ compared with controls (39.59 \pm 5.12 ). CRF made no significant change $(25.24 \pm 4.10)$. Forskolin (an activator of the CAMP secondary messenger pathway) also inhibits differentiation $(10.19 \pm 2.26, p$ $<0.05)$ compared with controls $(25.59 \pm 4.79)$. m-3M3FBS had no significant effect ( $17.11 \pm 5.38)$.

This work demonstrates a novel method for culturing osteoclasts obtained from human PBMCs. Ucn 1 and Ucn2 both showed an inhibitory effect on the differentiation of osteoclasts, where no such inhibition was observed with CRF. Ucn 1 inhibits differentiation of human osteoclasts through an increase in the activity of intracellular CAMP and not PLC. These results suggest a role for Ucn 1 in the inhibition of osteoclast action, implicating Ucn1 or downstream activators of the CAMP pathway as potential novel therapies for the reduction of excessive bone resorption experienced in osteoporosis.

\section{P60}

In vivo correlation of single-slice peripheral quantitative computed tomography (pQCT) and high-resolution pQCT measures at the tibia

\author{
Mícheál Ó Breasail, ${ }^{1}$ Ann Prentice, ${ }^{1}$ and Kate Ward ${ }^{1,2}$ \\ ${ }^{1}$ Elsie Widdowson Laboratory, MRC, Cambridge, UK \\ ${ }^{2}$ Lifecourse Epidemiology Unit, MRC, Southampton, UK
}

Objectives: Single-slice pQCT (pQCT) and high-resolution pQCT (HRpQCT) are available for in vivo measurements of cortical $(\mathrm{Ct})$ and trabecular ( $\mathrm{Tb})$ compartments. These techniques measure different sites, single-slice PQCT at any site along the bone and first-generation HRpQCT at distal sites. The aim was to explore 
the agreement between the methods for measures of cortical vBMD, cortical thickness, and trabecular vBMD.

Methods: The non-dominant tibias of 76 healthy premenopausal women (mean [SD] age 35.4 [3.6] years) were measured. Outcomes were: pQCT (XCT 2000L, voxel size $0.5 \mathrm{~mm}$ ) 4\% TbvBMD, $14 \%$ and $38 \%$ Ct-vBMD and thickness; HRpQCT (XtremeCTI, voxel size $0.82 \mu \mathrm{m}$ ) a $9.02 \mathrm{~mm}$ volume of interest (110 contiguous slices) at the distal tibia (Tb-vBMD, Dinner [Dinn], Ct-vBMD, and thickness). Correlations between comparable parameters were obtained, and Bland-Altman (BA) plots were used to assess the agreement between the two methods and to detect systematic bias.

Results: Ct-vBMD measures on $\mathrm{pQCT}$ and HRpQCT were poorfairly correlated $\left(R^{2}=0.17,0.37\right.$ for $38 \%$ and $14 \%$ tibia, respectively). There was good correlation between cortical thickness at $14 \%$ pQCT site and HRpQCT $\left(R^{2}=0.68\right)$ but not at the $38 \%$ tibia $\left(R^{2}=0.09\right)$. BA plots showed increasing systematic difference at both sites with increasing cortical thickness. There were high correlations between Tb-vBMD by $\mathrm{PQCT}$ and both HRpQCT Tb-vBMD and Dinn $\left(R^{2}=0.87,0.83\right.$, respectively). There was good agreement between the Tb outcomes; pQCT Tb-vBMD was lower than both HRpQCT Tb measures mean $(95 \% \mathrm{Cl})$ difference of Tb-vBMD $54 \mathrm{mg} / \mathrm{cm}^{3}\left(-26 ;-82 \mathrm{mg} / \mathrm{cm}^{3}\right)$ and Dinn $95 \mathrm{mg} / \mathrm{cm}^{3}\left(-62 ;-128 \mathrm{mg} / \mathrm{cm}^{3}\right)$.

Conclusion: Correlations between cortical vBMD and thickness measured at the proximal (38\% pQCT site) and distal (HRpQCT) tibia are weak. However, cortical measures at the $14 \%$ and distal site agreed well. Trabecular measures agreed well but cannot be interchanged. To understand cortical bone adaptations to environment, a proximal site should be used.

\section{P61}

Developing and testing novel delivery systems for glutamate receptor antagonists for the treatment of joint pain and disease

\section{Ben Egan, ${ }^{1,2}$ James Birchall, ${ }^{2}$ Charles Heard, ${ }^{2}$ and Deborah Mason ${ }^{1}$}

\author{
${ }^{1}$ School of Bioscience, Cardiff University, Cardiff, UK \\ ${ }^{2}$ School of Pharmacy and Pharmaceutical Sciences, Cardiff \\ University, Cardiff, UK
}

The AMPA/kainate glutamate receptor (GluR) antagonist NBQX reduced bone destruction and expression of bone turnover markers when injected intra-articularly, in rat antigen-induced arthritis (AIA). ${ }^{(1)} \mathrm{NBQX}$ is similarly protective in osteoarthritis rodent models. ${ }^{(2)} \mathrm{NBQX}$ also reduced mineralization in human primary osteoblasts in vitro. ${ }^{(1)}$

We are developing sustained release and progressively less invasive methods of GluR antagonist delivery, using the related drug DNQX, to improve therapeutic effect, patient comfort, and ease of application.

DNQX loaded Poly(lactic-co-glycolic acid) (PLGA) nanoparticles were synthesized via double emulsion. DNQX loaded thermosetting hydrogels were synthesized on ice by dissolving Pluronic-F127 (22\%w/v) and Carbopol $934(0.5 \% \mathrm{w} / \mathrm{v})$ in $\mathrm{dH}_{2} \mathrm{O}$, homogenizing with DNQX/NBQX $\left(2.5 \mathrm{mM}\right.$ in $\left.\mathrm{dH}_{2} \mathrm{O}\right)$ and set in dialysis cassettes for 5 minutes at $37^{\circ} \mathrm{C}$. PLGA nanoparticles and hydrogels were suspended in PBS at $37^{\circ} \mathrm{C}$ and supernatants analyzed using high-performance liquid chromatography (HPLC) to determine drug release rate.
Y201 MSCs were differentiated to osteoblasts $(20,000$ cells/well, DMEM $+10 \%$ FBS supplemented with Dexamethasone, BGlycerophosphate, and Ascorbic acid-2-phosphate) in the presence/absence of NBQX $(200 \mu \mathrm{M})$ and DNQX (200 and $400 \mu \mathrm{M})$. Mineralization was determined by quantification of Alizarin red staining.

PLGA nanoparticles encapsulated 28mM DNQX (encapsulation efficiency $=71 \%$ ) and released $21 \mathrm{mM}$ DNQX over 1 hour, followed by sustained release of remaining DNQX over the next 48 hours. Hydrogels released their $2.5 \mathrm{mM}$ DNQX load over 24 hours in $37^{\circ} \mathrm{C}$ PBS. Alizarin red staining was reduced by both DNQX $(0.56 \pm 0.14)$ and NBQX $(0.56 \pm 0.04)$ when compared with controls $(1.31 \pm 0.19)$ after 14 days $(n=3)$, indicating a repression of mineralization.

Intra-articular injection of NBQX reduces degeneration in arthritis rodent models. ${ }^{(1,2)}$ Sustained release offered by PLGA nanoparticles and hydrogels may improve efficacy and application ease of GluR antagonists. NBQX and DNQX reduced mineralization in Y201-derived osteoblasts, consistent with effects in human primary osteoblasts. ${ }^{(1)}$ This may partially explain the reduced bone remodelling observed in AIA after NBQX treatment, ${ }^{(1)}$ indicating bone-modifying potential for joint disease treatment.

\section{References}

1. Bonnet CS, Williams AS, Gilbert SJ, Harvey HK, Evans BA, Mason DJ. AMPA/kainate glutamate receptors contribute to inflammation, degeneration and pain-related behavior in inflammatory stages of arthritis. Ann Rheum Dis. 2015;74:242-51.

2. Bonnet CS, Mason DJ. BORS 2017 Meeting Abstract.

\section{P62}

Bone Research Society Barbara Mawer Travelling Fellowship update: investigating extracellular ATP in the tumor microenvironment of osteosarcoma using the plasma membrane-targeted luciferase (pmeLUC) probe

\section{Luke Tattersall, ${ }^{1}$ Elena De Marchi, ${ }^{2}$ Francesco Di Virgilio, ${ }^{2}$ Michelle Lawson, ${ }^{1}$ Elena Adinolfi, ${ }^{2}$ and Alison Gartland ${ }^{1}$}

\author{
${ }^{1}$ The Mellanby Centre for Bone Research, The University of Sheffield, \\ Sheffield, UK \\ ${ }^{2}$ Department of Morphology, Surgery and Experimental Medicine, \\ University of Ferrara, Ferrara, Italy
}

Osteosarcoma is the most common type of primary bone cancer affecting adolescents, attributed to rapid bone growth and turnover; it is a rare, incurable disease. The 5-year survival rate remains $65 \%$, which reduces to $20 \%$ with metastasis. This highlights the need for development of novel therapeutics to treat osteosarcoma. One potential avenue to explore in the setting of osteosarcoma treatment is purinergic signalling, involving extracellular nucleotides binding to purinergic receptors expressed in many cancers and present on osteoblasts. ATP is at a high concentration in the tumor microenvironment but absent from surrounding healthy tissue. This is particularly pertinent in the bone tumor microenvironment where mechanical loading stimulates ATP release. ATP release from osteosarcoma cells, which may depend on the P2 $\times 7 \mathrm{R}$ isoform expressed, could contribute toward disease progression. Current techniques that quantify ATP levels in biological systems are limited to measuring it in serum or cell supernatants, which may be orders of magnitude lower than the levels at the 
cellular surface. However, pmeLUC is a novel plasmid that allows the measurement of ATP concentrations at the cellular membrane. pmeLUC was produced by attaching membrane targeting sequences ( $\mathrm{N}$-terminal leader sequence and $\mathrm{C}$ terminal GPI anchor) derived from a folate receptor to a fulllength firefly luciferase sequence and was cloned into a pcDNA3 plasmid. Expression of this construct at the outer plasma membrane enables ATP released from cells to be detected in the immediate extracellular space. During a Bone Research Society Barbara Mawer Travelling Fellowship, pmeLUC was transfected into two osteosarcoma cell lines-MNNG-HOS and Te85. Successful transfections were confirmed by measuring ATP levels, and these cells are currently being used to assess the effect of ATP and P2 $\times 7 R$ isoforms on osteosarcoma both in vitro and in vivo. More sensitive and accurate quantification of ATP levels at the cell surface would better inform us about P2 receptor activation and improve our understanding of the role of purinergic signalling in the tumor microenvironment.

\section{Late-Breaking Poster Presentation Abstracts}

\section{LBP1}

Temporal investigation of tissue-engineered cartilage from human fetal skeletal progenitor cells using multimodal label-free imaging

\section{Catarina Costa Moura, ${ }^{1,2}$ Rahul S Tare, ${ }^{2,3}$ Richard OC Oreffo, $^{2}$ and Sumeet Mahajan ${ }^{1}$}

\section{${ }^{1}$ Institute for Life Sciences and Department of Chemistry, University of Southampton, Southampton, UK \\ ${ }^{2}$ Centre for Human Development, Stem Cells and Regeneration, Institute of Developmental Science, University of Southampton, Southampton, UK \\ ${ }^{3}$ Mechanical Engineering Department, Faculty of Engineering and the Environment, University of Southampton, Southampton, UK}

The use of skeletal progenitor cells in cell-based therapies is currently one of the most promising areas for skeletal disease treatment and skeletal tissue repair. The unique properties of skeletal progenitor cells, with their ability to self-renew and potential to differentiate along the stromal lineages, make skeletal progenitor cells an ideal cell population for bone and cartilage reparative medicine. Current limitations in the characterization of skeletal progenitor cell differentiation have led to the application of alternative strategies that aim to identify molecules at the subcellular level by using their inherent properties, without the use of a dye or label, ie, "label-free." Label-free methods such as coherent anti-Stokes Raman scattering (CARS) and second harmonic generation (SHG) microscopy are minimally invasive and non-destructive, and are emerging as powerful alternatives to conventional techniques in biomedicine. In this work, human fetal femur-derived skeletal cells were differentiated into cartilage in vitro using a 21-day 3D pellet culture system and examined using label-free methods. Typical biological components such as lipids and proteins were imaged using CARS and, for the first time, it was possible to image other cellular structures including glycosaminoglycans.

Elucidation of the architecture of the differentiated tissue is a key component for cartilage tissue engineering. The application of $3 \mathrm{D}$ in place of $2 \mathrm{D}$ imaging further enabled a more comprehensive understanding of the collagen fibrous network during the chondrogenic development of human fetal-femur derived skeletal cells. Quantitation of the various molecular and structural readouts allowed developing assessment parameters to track the differentiation of human skeletal progenitor cells and their in vitro tissue development.

In summary, the non-invasive nature of CARS and SHG imaging presents an advantageous alternative to characterize and monitor cartilage development from human fetal skeletal progenitor cells. Crucially, these studies demonstrate the immense value of non-linear multimodal imaging and labelfree approaches and their application to human skeletal repair and regeneration research.

This work was supported by the Institute for Life Sciences, University of Southampton, BBSRC (BB/L021072/1), ERC grant Nano-ChemBioVision 638258, and Wessex Medical Research.

\section{LBP2-Abstract withdrawn}

\section{LBP3}

Enrichment of bone progenitor populations from human bone marrow using label-free microfluidic techniques

Miguel Xavier, ${ }^{1}$ Stefan Holm, ${ }^{2}$ Jason Beech, ${ }^{2}$ Daniel Spencer, ${ }^{1}$ Jonas Tegenfeldt, ${ }^{2}$ Richard OC Oreffo, ${ }^{3}$ and Hywel Morgan

${ }^{1}$ Institute for Life Sciences, University of Southampton, Southampton, UK

${ }^{2}$ Solid State Physics, Lund University, Lund, Sweden

${ }^{3}$ Centre for Human Development, Stem Cells and Regeneration, University of Southampton, Southampton, UK

Skeletal stem cells (SSCs) reside in the human bone marrow (BM) and are responsible for the unique regeneration capacity inherent to bone. A current unmet challenge hampering their clinical translation remains the isolation of homogeneous SSC populations with consistent regeneration and differentiation capacities. Factors limiting the efficiency of existing sorting approaches include the complexity of BM, the scarcity of SSCs in BM, estimated at fewer than 1 in 10,000 cells, and the absence of a specific marker that is unique to the SSC.

Microfluidics offers the potential to characterize and sort cells based on intrinsic biophysical properties. Using impedance cytometry coupled to the fluorescence detection of Stro-1+/ CD146+ cells, the size and membrane capacitance of SSCs were determined from human BM samples. The membrane capacitance of SSCs was found to be indistinct from other cells in BM. Conversely, their average size in suspension, at 9 micrometers, was within the largest cell fraction in BM. The stiffness of SSCs was investigated using real-time deformability cytometry-a microfluidic technique for high-throughput screening of cell mechanical properties. Critically, SSCs were found to be significantly stiffer than white blood cells, which are abundant in human BM.

Centered on these findings, label-free sorting devices were designed, based on the principle of deterministic lateral displacement (DLD). DLD uses arrays of micropillars to sort cells based on their diameter, at thousands per second. Cell deformation, induced by shear and contact with the pillars, can change the effective cell size and affect sorting efficiency. This was demonstrated by sorting two human cells lines (MG-63 and $\mathrm{HL}-60)$ of different size and stiffness, achieving purities up to 
$98 \%$, and by size fractionation of expanded SSCs. Significantly, SSCs sorted by DLD remained viable and retained their capacity to form clonogenic cultures.

Overall, this work provides a detailed characterization of relevant biophysical properties of SSCs and paves the way toward the design of a novel label-free sorting approach based on DLD to provide purified SSC populations from BM with impactful use in fundamental stem cell research and the clinic.

\section{LBP4}

Does a hysterectomy predispose women to developing osteoarthritis?

\section{Jenny Martin, Caitlin Murphy, Jenny Gregory, Richard Aspden, Anna Riemen, and Fiona Saunders}

\section{Arthritis and Musculoskeletal Research, University of Aberdeen, Aberdeen, UK}

The anatomical shape of bones and joints is crucial to their function; however, detecting and quantifying pathological variations can be challenging. A dramatic increase in the prevalence in postmenopausal women has led to the suggestion that hormonal factors may play a role in the pathogenesis. This study aims to examine if undergoing a hysterectomy, both with retention and removal of ovaries, predisposes women to osteoarthritis (OA) and, secondly, if the development is influenced by hormone-replacement therapy (HRT). Statistical shape modelling (SSM) is a method of image analysis that allows for detection of subtle shape variation described by a set of landmark points. Through the generation of linearly independent modes of variation, each image can be described in terms of numerical scores. Using radiographs of 149 female participants from the Osteoarthritis Initiative (OAI), SSM was applied to examine how hip morphology differed in a cohort who had undergone hysterectomies compared with controls. There were no differences observed in BMI, age, height, or weight between groups. ANOVA and Games-Howell post hoc analysis showed that modes 3 and 5 were statistically significant. Lower mode 3 scores were associated with hysterectomy $(p=0.019)$. Lower mode 3 scores are associated with narrowing of the femoral neck and increased acetabular coverage. Lower mode 5 scores were associated with hysterectomy and oophorectomy $(p=0.049)$. Lower mode 5 scores were associated with reduced coverage of the femoral head, superolateral migration of the femoral head, and larger greater trochanter. There were no associations observed between HRT use and OA. The subtle morphologic features of hip OA present in only hysterectomized women thus suggests undergoing a hysterectomy may be a predisposing factor and a clinical consideration after surgery. The use of HRT was not observed to influence the development of $O A$ and thus cannot be suggested as a protective measure.

\section{LBP5}

Surgical menopause, hip shape and OA: are they related? Caitlin Murphy, Jenny Martin, Jenny Gregory, Richard
Aspden, Anna Riemen, and Fiona Saunders

Arthritis and Musculoskeletal Research, University of Aberdeen, Aberdeen, UK
Osteoarthritis (OA) is a degenerative disease of the joints and is associated with aging. The etiology of $O A$ is thought to be influenced by endogenous estrogen levels as there is an increase in prevalence in postmenopausal women. The aim of this study was to investigate the relationship between hysterectomy and OA through the application of statistical shape modelling (SSM) and, secondly, if the development is influenced by hormonereplacement therapy (HRT).

Hip radiographs from 173 women from the Osteoarthritis Initiative (OAI) were analyzed using SSM. A template consisting of 58 points was used to mark the shape of the proximal femur and acetabulum. Subjects were defined into the following categories: control, hysterectomy, hysterectomy and partial oophorectomy, and total hysterectomy.

There were no observed differences in age, height, weight, and BMI between the groups. The first 10 modes of shape variation were investigated. Pearson correlation indicated statistical significance between hysterectomy status and modes 2 $(p=0.024)$ and $5(p=0.013)$. Shapiro-Wilk test of normality indicated normal distribution of data. One-way ANOVA with Tukey post hoc test showed no statistical significance between hysterectomy status and any of the 10 modes. Similarly, there were no associations observed between HRT use and mode score.

SSM provided modes of shape variation that identified anatomical characteristics of $\mathrm{OA}$, although there was no evidence of statistically significant relationships in this data set.

\section{LBP6}

A novel MRI method for non-destructive vertebral strength quantification

Amanda Davies, ${ }^{1}$ Faizan Ahmad, ${ }^{2}$ Peter Theobald, ${ }^{2}$ Richard Hugtenburg, $^{3}$ and Richard Johnston ${ }^{4}$

${ }^{1}$ Osteotronix Ltd, Swansea, UK

${ }^{2}$ School of Engineering, Cardiff University, Cardiff, UK

${ }^{3}$ College of Medicine, Swansea University, Swansea, UK

${ }^{4}$ College of Engineering, Swansea University, Swansea, UK

Vertebral fractures are twice as common as any other fracture in osteoporosis (OP) patients and are typically a first indicator of the disease. However, the lack of a direct, non-invasive method to assess mechanical strength prevents prospective identification of vertebrae likely to fracture. Dual-energy X-ray absorptiometry (DXA) is the gold standard technique, calculating areal bone mineral density (aBMD), which is used with clinical factors to estimate fracture risk (NICE, 2012). DXA is limited by the overlap observed in $T$-scores in individuals with and without fracture (McCreadie $B, 2000$ ). A magnetic resonance imagingbased (MRI) technique, fineSA, has been reported that can noninvasively quantify fine structures (Evans BAJ, et al., 2012; Rafferty J, et al., 2016), and this study explores its potential to provide a more accurate measure of fracture resistance.

The $L_{1}$ to $L_{3}$ lumbar vertebrae were excised from deer of the same herd ( $n=11$ ) culled for the meat industry (approximately 24 months old). Each sample underwent the fineSA method (Siemens 3T Magnetom Skyra, Swansea University), micro-CT (Nikon XT H 225, Swansea University), DXA (Horizon A machine, Singleton Hospital), and compression testing (Instron, Cardiff University). Pearson's correlation coefficient was calculated and multiple regression performed using MATLAB (2011b). 
The fineSA metric was strongly correlated $(r=-0.86, p=0.003)$ with mechanical strength, and this relationship was stronger than that observed for $\operatorname{aBMD}(r=0.71, p=0.03)$. Non-significant correlations were observed between micro-CT measurements and mechanical strength. Multiple regression showed that adding the fineSA metric to aBMD in the model increases the percentage of variance in mechanical strength explained from $50 \%$ to $78 \%(p=0.01)$.

This study provides early evidence that fineSA could provide a more accurate method to predict fracture occurrence by closely approximating mechanical strength. Further work is required to progress toward clinical trials, with an initial application of this novel approach to potentially include oncology patients, where MRI is in routine use and an appreciation of mechanical strength has even greater significance.

\section{LBP7}

The $\triangle E 50-M D$ dog model of Duchenne muscular dystrophy has a skeletal phenotype

\section{Emma Wintsch, Richard Piercy, and Michael Doube}

\author{
${ }^{1}$ Skeletal Biology Group, Royal Veterinary College, London, UK \\ ${ }^{2}$ Comparative Neuromuscular Diseases Laboratory, Royal \\ Veterinary College, London, UK
}

Duchenne muscular dystrophy (DMD) is a fatal, X-linked disease caused by null-mutations in the dystrophin gene, which results in progressive striated muscle degeneration in boys. Dog models are used to evaluate treatments for DMD because dystrophin-null mice fail to display a clinically relevant phenotype. We reported a spontaneous DMD hotspot mutation in a Cavalier King Charles spaniel. ${ }^{(1)}$ The mutation has been preserved by breeding carrier females onto a Beagle background; affected dogs are termed $\triangle \mathrm{E} 50-\mathrm{MD}$. Since boys with DMD have a high fracture rate, we hypothesized that in comparison with unaffected male dogs, $\Delta$ E50-MD dogs have a skeletal phenotype. Left calcanei of 10 affected dogs and 5 unaffected male littermates were dissected after euthanasia at 5 to 30 months old and imaged with X-ray microtomography. BoneJ measured trabecular thickness (Tb.Th), trabecular spacing (Tb.Sp), connectivity density (Conn. D), degree of anisotropy (DA), and volume fraction (BV/TV) in volumes of interest containing trabecular bone, and cross-sectional area (CSA) and 2D thickness on whole cross sections along the entire length of each bone. Bone length was measured with digital calipers. Trabecular parameters and calcaneus length were compared between affected and unaffected dogs using Welch's or MannWhitney's $t$ test after normality testing. CSA and 2D thickness measurements were averaged within 20 bins, one per $5 \%$ of bone length. A two-way ANOVA was used to analyze withinand between-group effects. Trabecular parameters and bone length were not different between affected and unaffected dogs (BV/TV $p=0.164$; Tb.Th $p=0.181$; Tb.Sp $p=0.440$; Conn. $\mathrm{D} p=0.202$; DA $p=0.544$; length $p=0.070$ ), while strong, significant variations $(p<0.0001)$ were detected among length bins and between genotypes for 2D thickness and CSA. Affected dogs' calcanei had lower mean 2D thickness $(0.54 \pm 0.25$ versus $0.79 \pm 0.37 \mathrm{~mm})$ and smaller cross sections $\left(31.1 \pm 9.4\right.$ versus $\left.49.4 \pm 14.3 \mathrm{~mm}^{2}\right)$, with differences pronounced around 30-65\%, and 90-95\% length, which corresponds to the sustentaculum tali, talar articular surfaces, and calcaneal tuber. These areas coincide with the articular and tendinous function of the calcaneus. $\triangle \mathrm{E} 50-\mathrm{MD}$ dogs have a skeletal phenotype that may be useful in assessing bone outcomes of DMD therapies.

\section{Reference}

1. Walmsley GL, Arechavala-Gomeza V, Fernandez-Fuente $M$, et al. A Duchenne muscular dystrophy gene hot spot mutation in dystrophin-deficient Cavalier King Charles spaniels is amendable to exon 51 skipping. PLoS One. 2010;5(1):e8647.

\section{LBP8}

Undercarboxylated osteocalcin, but not the carboxylated form, may increase human aortic smooth muscle cell calcification

\section{Sophie Millar, Susan Anderson, and Saoirse O'Sullivan}

\author{
Graduate Entry Medicine and Medical Sciences, University of \\ Nottingham, Derby, UK
}

Objectives: Human studies report conflicting results on the association between osteocalcin, an osteoblast-derived hormone, and calcification. This research aimed to disentangle the roles of its major circulating forms within vascular calcification in vitro.

Methods: Confluent human aortic smooth muscle cells (HASMCs) were grown in 1 of 6 conditions for 21 days: normal growth media (control); normal growth media with $10 \mathrm{ng} / \mathrm{mL}$ undercarboxylated osteocalcin (ucOCN); normal growth media with $10 \mathrm{ng} / \mathrm{mL}$ carboxylated osteocalcin (cOCN); mineralization media (MM); MM with $10 \mathrm{ng} / \mathrm{mL}$ ucOCN; or MM with $10 \mathrm{ng} / \mathrm{mL}$ cOCN. Media was collected and cells were fixed and stained with Alizarin red for calcium at days $6,12,18$, and 21 . Total OCN secretion was measured by ELISA and alkaline phosphatase (ALP) activity was measured by colorimetric dephosphorylation of p-nitrophenyl phosphate. One-way ANOVAs with multiple comparisons were used to analyze differences between treatment groups.

Results: After 21 days, quantified calcium staining was significantly increased with all MM treatments compared with control $(n=2 ; p<0.01)$. ucOCN and $c O C N$ on their own did not induce calcification as measured directly by staining. MM with ucOCN resulted in more calcium staining than $\mathrm{MM}$ alone or $\mathrm{MM}$ with $\operatorname{cOCN}(n=2 ; p<0.05)$. At days 12,18 , and 21 , there was significantly more total osteocalcin secreted by cells treated with MM with ucOCN compared with control ( $n=2-4 ; p<0.05$ ). OCN was produced by MM-treated cells alone (about $30 \mathrm{ng} / \mathrm{mL}$ after 21 days) and at low concentrations in control cells (about $8 \mathrm{ng} / \mathrm{mL}$ ). At all time points, ALP activity was significantly increased with all MM treatments compared with those with control media $(n=2-4 ; p<0.01)$. ucOCN and $c O C N$ did not alter ALP activity in either control media treatments or MM treatments.

Conclusion: The main circulating forms of osteocalcin do not induce calcification on their own in physiological conditions after 21 days. In mineralizing conditions, ucOCN supplementation at physiological concentrations appeared to increase vascular calcification, while $\mathrm{COCN}$ did not, suggesting individual biological activity. Additionally, OCN is produced at low basal levels by HASMCs and is increased in differentiated and calcified cells. 


\section{LBP9}

Correlation between serum $\mathbf{2 5}$ hydroxyvitamin D, parathyroid hormone (PTH), and high-resolution peripheral quantitative computed tomography (HR-pQCT) parameters of the distal tibia

\section{Hassan A Alshamrani, ${ }^{1,2}$ Margaret A Paggiosi, ${ }^{1}$ Nick Bishop, ${ }^{1,2}$ and Amaka C Offiah ${ }^{1,2}$}

${ }^{1}$ Mellanby Centre for Bone Research, Department of Oncology and Metabolism, The University of Sheffield, Sheffield, UK

${ }^{2}$ Sheffield Children's NHS Foundation Trust, Sheffield, UK

Objectives: The aim of this study was to determine the strength of association between serum 25 hydroxyvitamin D, PTH, and HRpQCT parameters of the distal tibia in a young Middle Eastern/North African (MENA) population living in the United Kingdom (UK).

Methods: Forty apparently healthy young people who emigrated from the MENA countries to the UK ( 20 males, 20 females) were recruited. Serum 25 hydroxyvitamin D and PTH levels were determined between November 2017 and March 2018. All blood samples were collected in the morning after an overnight fast. The non-dominant distal tibia of all participants was scanned in order to assess bone microstructure using an XtremeCT HRpQCT scanner (Scanco Medical).

Results: The age of the participants ranged from 18 to 38 years (median $=28.1, \mathrm{SD}=7$ ). The mean total 25 hydroxyvitamin D was $27.8 \mathrm{nmol} / \mathrm{L}(\mathrm{SD}=19.6$, range $=85)$, while the mean PTH was $71.4 \mathrm{ng} / \mathrm{L} \quad(S D=33.5$, range $=170.50)$. The correlation results showed that there were weak but significant correlations between PTH and the following HR-pQCT parameters: total density $(r=0.364, p=0.024)$, inner trabecular density $(r=0.340$, $p=0.037)$, and trabecular bone volume/tissue volume $(r=0.331, p=0.042)$. Regression analysis showed that 1$)$ the factors which in combination were most strongly associated with cortical density were calcium intake and sex (adjusted $\left.\mathrm{R}^{2}=36.9 \%\right) ; 2$ ) the factors which in combination were most strongly associated with trabecular density were height, PTH, weight, and age (adjusted $\mathrm{R}^{2}=55.9 \%$ ); and 3 ) the factors which in combination were most strongly associated with cortical area were weight and sex (adjusted $\mathrm{R}^{2}=45.6 \%$ ).

Conclusion: Low levels of vitamin D were evident among this cohort. Despite the low levels of vitamin D, mean PTH was only slightly elevated among this ethnic group who live in the UK. The results of this correlation study indicate that PTH has a greater impact on trabecular bone microstructure than vitamin D. Height, weight, sex, age, calcium intake, and PTH were strong predictors of cortical density, trabecular density, and cortical area among this cohort.

\section{LBP10}

Effect of vitamin D and whole body vibration on highresolution peripheral quantitative computed tomography (HR-pQCT) parameters of the distal tibia (VibeD study)

\author{
Hassan A Alshamrani, ${ }^{1,2}$ Margaret A Paggiosi, ${ }^{1}$ Nick \\ Bishop, ${ }^{1,2}$ and Amaka C Offiah ${ }^{1,2}$ \\ ${ }^{1}$ Mellanby Centre for Bone Research, Department of Oncology and \\ Metabolism, The University of Sheffield, Sheffield, UK \\ ${ }^{2}$ Sheffield Children's NHS Foundation Trust, Western Bank, \\ Sheffield, UK
}

Objectives: There is lack of understanding of the combined effect of vitamin D and whole body vibration (WBV) on bone quality, particularly for some minor ethnic groups who live in Europe (eg, immigrants from the Middle East/North Africa [MENA] region). The aim of the VibeD study is to determine the effect of vitamin D and WBV on HR-pQCT parameters of the distal tibia in male and female volunteers who migrated from the MENA to the United Kingdom.

Methods: VibeD is a double-blind pilot randomized control trial with four parallel groups of equal numbers (40 in total; 20 males, 20 females). Group 1: WBV and vitamin D; group 2: WBV and placebo; group 3: vitamin D only; and group 4: placebo only. A single large oral dose of vitamin D $(150,000 \mathrm{IU})$ was administered after collecting baseline data including anthropometry, HRpQCT of the distal tibia, vitamin $\mathrm{D}$, bone profile, and parathyroid hormone (PTH). One week later, second void fasting urine samples were collected from all participants. The WBV consisted of three vibration sessions every week for 12 consecutive weeks. Ethical approval was granted.

Results: Forty apparently healthy volunteers aged 18 to 38 years (median $=28.1, \mathrm{SD}=7$ ) have been recruited. The calcium creatinine ratio ranged from 0.02 to $0.42 \mathrm{mmol} / \mathrm{mmol}$ creatinine (mean $=0.18, \mathrm{SD}=0.09$ ). To date (April 2018), 17 participants have completed the study. Preliminary analysis indicates statistically insignificant increases as follows: serum 25 hydroxyvitamin D (+6.37 nmol/L); serum PTH (+16.1 ng/L); total bone density $\left(+1.025 \mathrm{mg} / \mathrm{cm}^{3}\right)$; cortical density $\left(+1.63 \mathrm{mg} / \mathrm{cm}^{3}\right)$; and trabecular density $\left(+0.81 \mathrm{mg} / \mathrm{cm}^{3}\right)$. However, there was a significant decrease in corrected calcium $(-0.071 \mathrm{mmol} / \mathrm{L}$, $p=0.03)$ and a significant increase in alkaline phosphatase $(+3.8 \mathrm{U} / \mathrm{L}, p=0.023)$.

Conclusion: The high dose of administered vitamin D appears to be safe in this population. Preliminary results show statistically insignificant changes in vitamin $D$ and bone density, which may become significant in a larger study population. The study is ongoing and subgroup analysis will be possible once all data have been collected.

\section{LBP11}

Probing the skeletal stem cell niche through functional investigation of Prx1-expressing cells

\author{
Sarah Pretorius, ${ }^{1}$ Scott Roberts, ${ }^{2}$ and Malcolm Logan ${ }^{1}$ \\ ${ }^{1}$ Randall Division of Cell and Molecular Biophysics, King's College \\ London, London, UK \\ ${ }^{2}$ UCB Pharma, Slough, UK
}

The periosteum is the outer layers of cells covering the bone and is essential for appositional bone growth, fracture repair, and maintenance of healthy bone. Despite its importance, little is known about development and maintenance of the periosteum and how it carries out its various functions.

Prx 1 expression marks an early population of mesenchymal cells in the developing limb buds and later in the adult becomes restricted to a subpopulation of cells within the periosteum. This subpopulation of cells was isolated using FACS and found to be osteogenic and chondrogenic in culture, as well as contributing to the fracture callus in vivo. We are studying the function and properties of these periosteumderived cells (PDCs) using a novel Prx1eGFP reporter transgenic mouse, both in vivo and in vitro. Understanding 
the roles of PDCs will be fundamental in targeting these cells for the generation of therapies for bone disorders such as osteoporosis.

\section{LBP12}

Computed tomography-based texture analysis improves the prediction of incident vertebral fracture

\section{Fjola Johannesdottir, ${ }^{1,2}$ Brett Allaire, ${ }^{1}$ Dennis E Anderson, ${ }^{1,2}$ Elizabeth J Samelson, ${ }^{3,4,5}$ Douglas P Kiel, ${ }^{3,4,5}$ and Mary L Bouxsein $^{1,2}$}

${ }^{1}$ Center for Advanced Orthopedic Studies, Beth Israel Deaconess Medical Center, Boston, MA, USA

${ }^{2}$ Orthopedic Surgery, Harvard Medical School, Boston, MA, USA

${ }^{3}$ Institute for Aging Research, Hebrew Senior Life, Roslindale, MA, USA

${ }^{4}$ Medicine, Beth Israel Deaconess Medical Center, Boston, MA, USA

${ }^{5}$ Medicine, Harvard Medical School, Boston, MA, USA

Objectives: Although vertebral fractures (VF) are common, the current clinical standard for VF fracture risk assessment, bone mineral density (BMD) by DXA, is inadequate. Less than half of adults who fracture have osteoporosis according to their BMD. The purpose of this study was to determine if co-occurrence textural features (Haralick 1973) from computed tomography (CT) images combined with BMD predict incident VF better than BMD alone.

Methods: We conducted a case-control study of 22 incident VF cases (11 men, 11 women) and 54 age- and sex-matched controls aged 50 to 85 years, selected from the Framingham multi-detector computed tomography cohort. Textural analysis was performed on a volume of the $L_{3}$ trabecular bone incorporating the middle $11.6 \mathrm{~mm}$ of vertebral body height. Gray level co-occurrence matrices were calculated (distance of 1 voxel in each neighboring direction) and the Haralick textural features were derived from the matrices to quantify textural patterns. We also measured $L_{3}$ trabecular vBMD and DXA-equivalent aBMD from the CT scans. The prediction of incident VF was evaluated using conditional logistic regression analysis including age, height, and weight as covariates. The model discriminative capability was assessed with the area under the receiver operating characteristic (ROC) curve (AUC).

Results: We found that combining $L_{3}$ DXA-equivalent and two textural features (sum average and difference variance) improved the fracture prediction over aBMD alone $(p=0.02)$. ROC analysis showed that aBMD yielded AUC of 0.77 , whereas a combination of aBMD and the two textural features yielded a significantly higher AUC of 0.87. AUC for trabecular VBMD alone was 0.74 and was significantly higher when textural features were added to trabecular vBMD ( $A U C=0.86, p=0.01$ versus trabecular vBMD AUC alone). These findings imply that VF is associated with decreased image brightness (sum average) and increased local heterogeneity (difference variance).

Conclusion: Although limited by a small number of individuals with VF, these results suggest that incorporation of textural features from clinical CT may enhance VF prediction. Larger studies are needed to confirm these results.

Supported by NIH (R01 AR053986) and Framingham Heart Study (NIH/NHLBI Contract N01-HC-25195).

\section{LBP13}

A rare case of neck of femur fracture in a female adolescent associated with minor trauma and impaired bone metabolism

\section{Olamide Olatokun, Thomas Nash, and Hani B Abdul-Jabar}

Trauma \& Orthopaedics, Northwick Park Hospital, London, UK

This report describes a rare neck of femur (NOF) fracture in a young female after minor trauma without preceding history of disease.

Presenting Problem: A 17-year-old Somalian female presented with right hip pain after a fall down two steps. She had no past medical history, however weighed $42.4 \mathrm{~kg}, \mathrm{BMI} 15.82$. She had menarche at age 14 years with regular menstrual cycle. Skin was covered for cultural reasons.

Pelvic radiograph confirmed right NOF fracture. Given the lowimpact injury and rarity of NOF fractures in this age, extensive blood investigations were performed. Vitamin D was $2 \mathrm{nmol} / \mathrm{L}$ (50-140), calcium $1.92 \mathrm{mmol} / \mathrm{L}$ (2.20-2.60), parathyroid hormone (PTH) $14.2 \mathrm{pmol} / \mathrm{L}$ (1.6-6.9), phosphate 1.06 (0.80-1.50), ALP 380 IU/L (0-187). Others were normal. She was symptomatic of hypocalcemia, with positive Chvostek's sign.

Diagnosis was secondary hyperparathyroidism (SHPT) due to vitamin D deficiency (VDD), causing NOF fracture.

Clinical Management: Fracture was repaired with cannulated screws. A course of cholecalciferol was commenced. Follow-up radiographs were satisfactory, with DXA scan pending. Longterm dietary, orthopedic, and endocrinology team follow-up is required.

Discussion: Few cases of NOF fracture in children and adolescents are reported. Those reported are associated with significant trauma or if atraumatic, a case of gradually worsening hip pain is described. Here NOF fracture with a multifactorial etiology is presented, combining VDD and SHPT with minor trauma in a patient with low BMI.

Normal bone metabolism is complex, involving vitamin D, calcium, and PTH. Vitamin D maintains calcium levels by increasing intestinal calcium absorption. PTH stimulates conversion of vitamin $D$ to its active form, in turn increasing renal calcium resorption. Faults impair bone metabolism. Data suggest that impaired bone metabolism combined with VDD are important factors in NOF fracture pathophysiology. SHPT associated with increased fracture risk has been demonstrated. Parathyroidectomy for SHPT was shown to decrease hip fracture risk.

Vitamin D is vital for bone health. Here, it is stressed that VDD, particularly in adolescents, should not be overlooked as complications can follow. Early identification and supplementation is recommended.

\section{References}

1. Moten M, Mussa M, Naqvi S, Kulkarni S. A rare case of a nontraumatic neck of femur fracture in a 17-year-old boy associated with vitamin D deficiency. BMJ Case Rep. 2017;2017:pii: bcr2017-219385.

2. Dhar D. Bilateral traumatic fracture of neck of femur in a child: a case report. Malaysian Orthop J. 2013;7(2):34-6.

3. Orthobullets. Normal bone metabolism. 2018. Available at: https://www.orthobullets.com/basic-science/9012/normalbone-metabolism. Accessed April 20, 2018.

4. Seitz S, Koehne T, Ries C, et al. Impaired bone mineralization accompanied by low vitamin $D$ and secondary 
hyperparathyroidism in patients with femoral neck fracture. Osteoporos Int. 2013;24(2):641-9.

5. Madsen $C$, Jørgensen $H$, Lind B, et al. Secondary hyperparathyroidism and mortality in hip fracture patients compared to a control group from general practice. Injury. 2012;43(7):1052-7. 6. Isaksson A, Ivarsson $K$, Akaberi $S$, et al. The effect of parathyroidectomy on risk of hip fracture in secondary hyperparathyroidism. World J Surg. 2017;41(9):2304-11.

\section{LBP14}

The role of canonical and non-canonical autophagy in bone resorption by osteoclasts

\author{
Anh Tran, ${ }^{1}$ Sandra Segeletz, ${ }^{2}$ Emma McDermott, ${ }^{1}$ Justin \\ Rochford, ${ }^{3}$ Tom Wileman, ${ }^{4}$ and Miep Helfrich ${ }^{1}$ \\ ${ }^{1}$ Institute of Medical Sciences, University of Aberdeen, Aberdeen, UK \\ ${ }^{2}$ Max Planck Institute of Molecular Cell Biology and Genetics, MPI, \\ Dresden, Germany \\ ${ }^{3}$ Rowett Institute, University of Aberdeen, Aberdeen, UK \\ ${ }^{4}$ Biomedical Research Centre, University of East Anglia, Norwich, UK
}

LC3, a marker of autophagic vesicles, localizes to the osteoclast ruffled border (RB), suggesting a potential role of autophagy in osteoclastic resorption.

In vitro, $25 \%$ of osteoclasts from mice deficient in autophagy, through FIP200 deletion, still expressed GFP-LC3 at the RB. Live cell imaging demonstrated that GFP-LC3 localized to the RB in a time-dependent manner, slowly dissipating as the RB matured. The localization of LC3 to the RB may be analogous to LC3associated phagocytosis (LAP) in macrophages, an autophagyindependent pathway whereby LC3 is recruited to phagosomes to enhance phagosome maturation. In mice, autophagy deficiency through FIP200 deletion did not significantly affect resorption or bone density.

LAP is critically dependent on the proteins Rubicon and ATG16L1 (WD40 domain, specifically). We demonstrated that osteoclasts generated from the RAW264.7 cell line resorb mineralized dentine through RB formation. siRNA knockdown of Rubicon in RAW264.7-derived osteoclasts had no effect on canonical autophagy and resulted in modestly increased (13\%) resorption compared with controls. Live cell imaging with LysoTracker and GFP-Ezrin showed no difference in lysosomal vesicle targeting to the RB and in sealing zone (SZ) dynamics. Osteoclasts generated from mice with a deletion in the WD40 domain of ATG16L1, critical for LAP, showed no difference in canonical autophagy. The osteoclasts showed a mild increase in bone resorption in vitro. This was correlated with wider SZs and wider resorption trails than controls. Live cell imaging demonstrated the transgenic osteoclasts had more static SZs with lysosomes targeted to this region compared with the more dynamic SZs in controls.

Overall, our data demonstrate that both canonical and noncanonical pathways are involved in osteoclastic resorption. The data also demonstrate that live cell imaging is a useful tool to reveal more subtle effects on resorption caused by defective lysosomal targeting and by differences in SZ dynamics and size.

\section{LBP15}

Pleckstrin homology domain containing protein family member 1 (PLEKHM1) regulates bone resorption through sealing zone dynamics and lysosomal targeting in osteoclasts
Anh Tran, ${ }^{1}$ Emma McDermott, ${ }^{1}$ Justin Rochford, ${ }^{2}$ and Miep Helfrich ${ }^{1}$

${ }^{1}$ Institute of Medical Sciences, University of Aberdeen, Aberdeen, UK ${ }^{2}$ Rowett Institute, University of Aberdeen, Aberdeen, UK

Objectives: PLEKHM1 is implicated in the lysosomal fusion machinery in autophagic and endocytic pathways. Loss-offunction mutations in PLEKHM1 cause osteopetrosis in the ia/ia rat and in humans. We here examined the cellular consequences in osteoclasts of PLEKHM1 loss-of-function in mutant mice with a premature STOP codon at amino acid R714 (R714 STOP), resulting in the expression of a truncated non-functional protein.

Methods: Osteoclasts were generated from bone marrow and spleen cells of heterozygous (control) and homozygous (R714STOP) mutant mice and cultured on glass or dentine. Resorption pits on dentine were quantified by reflected light microscopy. Live cell imaging by spinnning-disk confocal microscopy was done with an actin probe (SiR actin) and lysosomal probe, LysoTracker, to identify polarized osteoclasts with a sealing zone (SZ), and lysosomal vesicles, respectively. Bone volume was assessed by micro-CT.

Results: R714STOP osteoclasts cultured on plastic showed no defects in autophagy. Polarized osteoclasts on dentine showed a significant decrease in resorption in vitro, but no defects in RB morphology were observed in vivo. Live cell imaging illustrated that osteoclasts exhibited three predominant SZ behaviors: 1) a stable SZ, which was typically crescent shaped and present for the duration of the imaging period, 2) a dynamic SZ, which would form and reform multiple times or undergo fusion and fission, or 3) a SZ that underwent just 1 cycle of SZ assembly and disassembly. In Het R714STOP osteoclasts, 39\% of osteoclasts had stable SZs, while only $26 \%$ of R714STOP osteoclasts exhibited this behavior. R714STOP osteoclasts predominantly had a dynamic SZ (49\%), which was associated less with a resorption pit. Only $34 \%$ of R714STOP osteoclasts were able to target LysoTracker to the RB (Het R714STOP: 53\%). In line with our in vitro analysis, R714STOP mice had an osteopetrotic bone phenotype.

Conclusion: PLEKHM1 deficiency in the R714STOP mouse leads to osteopetrosis, not through defects in RB formation but by a decrease in the targeting of lysosomes to the RB and a decrease in the number of osteoclasts with stable SZs.

\section{LBP16-Abstract withdrawn}

\section{LBP17}

Specific analysis of osteoclast-mediated bone resorption by differentiation of primary human osteoclasts in 3D

\section{Helen Knowles}

NDORMS, University of Oxford, Oxford, UK

Objectives: Osteoclasts are specialized bone-resorbing cells that drive the pathogenic osteolysis characteristic of varied conditions including rheumatoid arthritis (RA), osteosarcoma, and multiple myeloma. The presence of osteoclasts within diseased tissue is commonly associated with aggressive disease and poor prognosis. In vitro investigation of factors affecting osteoclast activity is complicated by the inability to easily 
distinguish from effects on differentiation, which must be performed in situ on the resorption substrate. We have therefore developed an alternative 3D method of osteoclast differentiation, which allows specific investigation of the bone resorption capacity of mature osteoclasts when re-seeded in 2D culture.

Methods: $\mathrm{CD} 14+$ monocytes were selected from peripheral blood and seeded into $2 \mathrm{mg} / \mathrm{mL}$ collagen gels in 24-well plates at $1 \times 10^{6} \mathrm{CD} 14+\mathrm{MON}$ per gel. Osteoclastogenesis was initiated by application of M-CSF and RANKL for 8 days. Mature osteoclasts were released from the gel by digestion with collagenase I and re-seeded onto dentine discs for analysis of resorption (toluidine blue staining).

Results: Similar rates of osteoclast formation were observed in 2D and 3D culture by bright-field microscopy. Once released and re-seeded, the final size of the osteoclasts formed with respect to number of nuclei was also similar. Mature osteoclasts re-seeded onto dentine discs were able to resorb bone and responded to resorption stimuli such as hypoxia $\left(2 \% \mathrm{O}_{2}, 24\right.$ hours, 2.6-fold induction, $p<0.05$ ) and HIF stabilization (PHD enzyme inhibition, 25 mM FG4592, 2.1-fold induction, $p<0.05$ ).

Conclusion: Differentiation of osteoclasts in $3 \mathrm{D}$ collagen gels allows direct re-seeding of mature osteoclasts onto dentine discs for specific analysis of bone resorption activity.

This project was funded by an Arthritis Research UK Career Development Fellowship (MP/19200) and the Rosetrees Trust (A1726).

\section{LBP18}

Altered expression of $\mathrm{A3}$ and $\mathrm{P2} \times 6$ receptors in osteocytes and chondrocytes after mechanical loading-novel mechanically regulated pathways

\author{
Amelia Redman, ${ }^{1}$ Sophie Gilbert, ${ }^{2,4}$ Carole Elford, ${ }^{1,2,4}$ \\ Ayesha Al-Sabah, ${ }^{3}$ Emma Blain, ${ }^{2,4}$ and Bronwen Evans ${ }^{1,4}$ \\ ${ }^{1}$ School of Medicine, Cardiff University, Cardiff, UK \\ ${ }^{2}$ School of Biosciences, Cardiff University, Cardiff, UK \\ ${ }^{3}$ Medical School, Swansea University, Swansea, UK
} ${ }^{4}$ Arthritis Research UK Biomechanics and Bioengineering Centre,
Cardiff University, Cardiff, UK

Mechanical loading of bone and cartilage maintains healthy tissues, and abnormal loading leads to disease. The mechanisms involved are poorly understood, and even though the purinergic receptor family has been implicated, only a few family members have been studied in this context. We investigated the effects of mechanical loading on $\mathrm{A} 3$ and $\mathrm{P} 2 \times 6$ receptor $(\mathrm{R})$ expression in in vitro $3 \mathrm{D}$ bone and cartilage loading models.

Samples were loaded using an ElectroForce $3200^{\circ}$ device. Murine IDG-SW3 osteocytes in 3D collagen gels were loaded at $2.5 \mathrm{~N}$ (physiological) or $5 \mathrm{~N}$ (pathophysiological) for 5 minutes/ $10 \mathrm{~Hz}$. Bovine cartilage explants obtained from metacarpophalangeal joints underwent loading at $2.5 \mathrm{MPa}$ or $7 \mathrm{MPa}$ for 15 minutes $/ 1 \mathrm{~Hz}$. Unloaded cells/explants were used as controls. RNA was extracted at 0.5 and 48 hours from osteocytes $(n=4 /$ loading condition/time point) and 1 and 24 hours from cartilage ( $n=6 /$ loading condition/time point) post-loading and relative expression of receptors determined (RT-qPCR). Protein expression was determined by IHC.

A3R expression in osteocytes was decreased after physiological loading at 48 hours $(p=0.004)$. In cartilage, A3R was expressed in controls but was absent in samples loaded at both $2.5 \mathrm{MPa}$ and $7 \mathrm{MPa}$ at 24 hours post-loading in one assay. Similar results were observed in a repeat assay, except $A 3 R$ expression occurred in two of the six samples at $2.5 \mathrm{MPa}$ and one sample at $7 \mathrm{MPa}$. A3R protein was present in unloaded cartilage and loaded samples are currently being analyzed. $\mathrm{P} 2 \times 6 \mathrm{R}$ expression was unaltered in loaded samples in osteocytes. However, cartilage showed a decrease in $\mathrm{P} 2 \times 6 \mathrm{R}$ expression in samples loaded at $7 \mathrm{MPa}$ at 24 hours post-loading $(p=0.014)$ correlating with previous mRNA array data.

These results provide novel information regarding purinergic signalling (A3R, P2 $\times 6 R$ ) and mechanically regulated pathways in bone and cartilage. Increased understanding of these pathways may help elucidate the pathogenesis of osteodegenerative diseases associated with adverse loading environments. 


\section{A}

Abdul-Jabar, Hani B - LBP13

Adams, Judith - OC7

Adinolfi, Elena - P62

Adoum, Anne-Tounsia - P18 \& P19

Ahlfeld, Tilman - P14

Ahmad, Faizan - LBP6

Ahmed, Faisal - OC17

Akune, Toru - P16

Al Muderis, Munjed - P56 \& P57

Allaire, Brett - LPB12

Allen, Rebecca - P19

Al-Sabah, Ayesha - LBP18

Al-sari, Usama A - P9*

Alshamrani, Hassan A - LBP9* \& LBP10*

Anderson, Dennis E - LPB12

Anderson, Susan - LBP8

Arden, Nigel - P58

Arnold, Emily - P49*

Aspden, Richard - OC7, LBP4 \& LBP5

Athanasou, Nick - P8

Ayre, Wayne - P36

Ayyalusamy, Ramamoorthy - P40

\section{B}

Baker, Andrew - P23

Barker, Alan - OC6

Barr, Rebecca - OC7

Bassett, JH Duncan - OC2, OC10, P13, P18, P21 \& P22

Bastin, Sonja - LB2

Beamish, Rebecca - P36*

Beech, Jason - LBP3

Behmoaras, Jacques - OC10

Ben-Shlomo, Yoav - OC1

Bhimjiyani, Arti Gauvri - OC1*

Birchall, James - P61

Bishop, Nicholas - OC5, LBP9 \& LBP10

Blain, Emma - LBP18* \& P26

Bolland, Mark - LB2

Bonnet, Cleo - OC9 \& P26

Bou-Gharios, George - P2

Bountra, Chas - OC11

Bourne, Lucie E - P44*

Bouxsein, Mary L - LPB12

Boyde, Alan - OC14, OC19 \& P25*

Bradley, Mark - P48

Brassett, Cecilia - P42

Brooke-Wavell, Katherine - P54

Browne, Martin - P53

Burska, Agata - P20

Butterfield, Natalie C - P13, P18*, P19 \& P22

\section{C}

Chantry, Andrew - OC3* \& P17*

Chen, Jiye - P46*

Chua, Melissa Y - P10

Cidonio, Gianluca - P14*, P47 \& P48

Clark, Emma - P9

Clarke, Shane - OC4
Clarkin, Claire - OC14 \& P28

Clynes, Michael - P30*

Cooper, Cyrus - OC4, OC16, OC8, P1, P30, P34, P4, P43, P5 \& P6

Cooper, Rachel - OC7

Cornish, Jillian - OC13*

Costa Moura, Catarina - LBP1*

Cosulich, Carlotta - P32

Coxon, Joshua - P59

Crabtree, Nicola - P45

Cronin, Rose-Marie - P26

Croucher, Peter I - OC2, P13, P21 \& P22

Crozier, Sarah - OC5

Curry, Katherine F - P22

Curtis, Elizabeth - OC5*

Czekańska, Ewa - P48

D

D'Angelo, Stefania - OC5

Davies, Amanda - LBP6*

Dawson, Jonathan - P1, P15, P47, P48 \& P55

De Andres, Maria C - OC11*

De Marchi, Elena - P62

Delmestri, Antonella - P1

Dennison, Elaine - OC8, P30, P34, P4 \& P5

Dewhurst, Hannah F - P19

D'Haese, Patrick - P44

Di Virgilio, Francesco - P62

Dickinson, Alex - P53

Dillon, Scott - P11*

Dodhia, Vikash H - P47*

Doube, Michael - LBP7, P10*

Down, Jenny - OC3 \& P17

Duggal, Niharika - OC8

Dunlop, Douglas - OC12

Dunning, Lynette - OC18

Dyke, Gareth - P52

\section{E}

Ebeling, Peter - P27

Eccles, Emily - OC9

Edwards, James R - P58

Edwards, Mark - P34 \& P5*

Egan, Ben - P61*

Elford, Carole - LBP18

El-Jawhari, Jehan - P20

Endo, Naoto - P35

Ersek, Adel - P32

Evans, Bronwen - LBP18

Evans, David - OC15

Evans, Holly - OC3 \& P17

Evans, Sam - P36

Evelyn-Wright, Stephanie - P53*

$\mathbf{F}$

Farquharson, Colin - OC17, P11, P24 \& P38

Felder, Alessandro A - P10

Ferrand, Rashida - P45

Ferrara, Napoleone - OC14, P28 
Fisher, Matt - P17

Folland, Jonathan - P54

Fordham, Richard - OC4

Fraser, Robert - OC5

Fraser, William - OC15

Fuggle, Nicholas - OC8* \& P34*

Fujisawa, Junichi - P35

\section{G}

Gallagher, James - OC19, P2 \& P41

Gamble, Gregory - LB2

Ganguly, Payal - P20*

Garratt, Liz - LB2

Gartland, Alison - P62

Gelinsky, Michael - P14

Geng, Jin - P48

Ghirardello, Elena J - P19 \& P22*

Giannoudis, Peter - P20

Gilbert, Sophie - OC9*, P26* \& LBP18

Gittoes, Neil - OC4

Glinka, Michael - P14 \& P48*

Gogakos, Apostolos - P13 \& P21

Gohin, Stephanie - P51*

Goodyear, Carl - OC18*

Goriainov, Vitali - OC12

Goring, Alice - OC14* \& P28

Gossiel, Fatma - OC5

Gostling, Neil J - P52

Gracia-Marco, Luis - OC6

Green, Alanna - OC3 \& P17

Greenwood, Charlene - P49 \& P50

Gregory, Jenny - OC7, LBP4 \& LBP5

Gregson, Celia L - OC1, OC15 \& P45

\section{H}

Halewood, Katherine - P38

Hardy, Rebecca - OC7

Hart, Deborah - P58

Hartley, April - OC15* \& P45*

Hartley, Chris - P54*

Harvey, lan - OC4

Harvey, Nicolas - OC4, OC16 \& P30

Hashizume, Hiroshi - P6

Heard, Charles - P61

Heawood, Ali - OC4

Helfrich, Miep - LBP14 \& LBP15*

Hesse, Eric - OC14

Hodges, Stephen - P51

Hohenstein, Peter - P23

Holland, Richard - OC4

Holm, Stefan - LBP3

Hooper, Kirsty - P38

Hopkinson, Mark - P51

Horii, Chiaki - P16

Horne, Anne - LB2

Horwood, Nicole - P32*

Howat, David - P51

Howe, Amanda - OC4

Hsu, Shun-Neng - P24*

Hudson, Katie - P17

Huesa, Carmen - OC18
Hughes, Andrew - P2

Hughes, Juliette - P2*

Hugtenburg, Richard - LBP6

Hung, John - P23

Hutchinson, John R - P10

I

Ignelzi, Michael A Jr - P39

Ihenacho, Menna - P26

lidaka, Toshiko - P16

Inglis, Stefanie - P12

Inskip, Hazel - P43

Ireland, Alex - OC7*

Ishimoto, Yuyu - P6*

Ismail, Omar - P59*

J

Jameson, Karen - P30 \& P34

Javaheri, Behzâd - P28* \& P51

Javaid, Muhammad - P1*

Jeffery, Nathan - OC19

Johannesdottir, Fjola - LPB12*

Johansson, Helena - OC16

Johnston, Richard - LBP6

Jones, Elena - P20

Jones, Rebecca - P59

Jones, Tim - P29*

Judge, Andrew - P43

\section{K}

Kaczynski, Jakub - P23

Kadamban, Premila - P41*

Kanczler, Janos - P12* , P14 \& P15

Kanis, John - OC4 \& OC16

Karabetsos, Matthew - P40

Karsenty, Gerard - P23

Kawaguchi, Hiroshi - P16

Kemp, John - OC15

Kennedy, Stephen - OC5

Kerslake, Robert - P54

Kiel, Douglas P - LPB12

Kijima, Yasufumi - P35

Kim, Yang-Hee - P14, P47, P48 \& P55*

Kłosowski, Michał M - P10

Kluzek, Stefan - P58

Knowles, Helen - LBP17* \& P8

Ko, Jeong-Hun - OC10

Kodama, Rie - P16

Kohn, David H - P40

Komla Ebri, Davide - P13 \& P21*

Kondo, Naoki - P35

Kuh, Diana - OC7

L

Lanham, Stuart - P14 \& P15

Larrouture, Quitterie - P8*

Lath, Darren - OC3 \& P17

Lawrence, Kevin - P59

Lawson, Michelle - OC3, P17 \& P62

Leitch, Victoria D - OC2* , P13, P18, P19 \& P22 
Lelliott, Christopher J - OC2

Lenaghan, Elizabeth - OC4 \& OC16

Leyland, Kirsten - P43

Lim, Khoon - P48

Litwic, Anna - P4

Liu, Ke - P2

Lockhart, John - OC18

Lodhia, Kalyani - P10

Logan, John G - OC2, OC19, P13, P18, P19, P21 \& P22

Logan, Malcolm - LBP11

Lord, Janet - OC8

Lu, William - P56* \& P57*

Luo, Jin - P46

\section{M}

MacRae, Vicky - OC17, P23 \& P24

Madhusudan, Namrata - OC11

Mahajan, Sumeet - LBP1

Majonga, Edith - P45

Mandair, Gurjit S - P39* \& P40*

Mannan, Naila S - P19*

Marquez-Grant, Nicholas - P50

Marshall, Tarnya - OC4

Martin, Jenny - LBP4* \& LBP5

Martin, Kathryn - OC7

Maslin, Kate - OC5

Mason, Debbie - P26

Mason, Deborah - OC9 \& P61

Mavrogordato, Mark - P53

McCloskey, Eugene - OC4 \& OC16*

McCulloch, Kendal - OC18

McDermot, Emma - LBP14, LBP15

McEwan, Josephine - OC12* \& P15*

McGivern, Hannah - P50*

McHugh, Grace - P45

McMillan, Lachlan - P27

Meeson, Richard - P51

Mihov, Borislav - LB2

Millar, Sophie - LBP8*

Mills, David - P25

Milne, Elspeth - OC17 \& P38

Miszkiewicz, Justyna - P18 \& P22

Moon, Rebecca - OC5

Morgan, Hywel - LBP3

Morgan, Stephanie - P38*

Morris, Michael D - P39 \& P40

Morton, Nicholas - OC17

Mujuru, Hilda - P45

Mukwasi, Cynthia - P45

Muraki, Shigeyuki - P16 \& P6

Murphy, Caitlin - LBP4 \& LBP5*

Muthuri, Stella - OC7

\section{$\mathbf{N}$}

Nakamura, Kozo - P16

Nash, Thomas - LBP13

Neuburger, Jenny - OC1

Neven, Ellen - P44

Newby, David - P23

Norman, Kingsley - OC9

Novak, Amanda - P24

Ntani, Georgia - P6
Nudelman, Fabio - P11

0

Ó Breasail, Mícheál - LB1* \& P60*

Offiah, Amaka C - LBP9 \& LBP10

Oka, Hiroyuki - P16

Olatokun, Olamide - LBP13*

Olsen, Bjorn - OC14 \& P28

O'Neill, TW - OC4

Oppermann, Udo - OC11

Oreffo, Richard - OC11, OC12, OC14, P12, P14, P15, P28, P47,

P48, P52, P55, LBP1 \& LBP3

Orriss, Isabel R - OC17 \& P44

Ossipov, Dmitri - P55

O'Sullivan, Saoirse - LBP8

$\mathbf{P}$

Padley, Jessica - P3

Paggiosi, Margaret A - LBP9 \& LBP10

Papageorghiou, Aris - OC5

Parsons, Camille - OC4* \& P43*

Patel, Jessal J - P44

Paternoster, Lavinia - OC15

Paton-Hough, Julia - OC3 \& P17

Pavlova, Anastasia - OC7

Pereira, Marie - OC10*

Peters, Tim - OC4

Petretto, Enrico - OC10

Piercy, Richard - LBP7

Pinedo-Villanueva, Rafael - P1

Pitsillides, Andrew - OC14, P28 \& P51

Pollard, Andrea S - P13* \& P19

Ponchel, Frederique - P20

Poole, Kenneth - P3, P35, P42 \& P7

Prentice, Ann - LB1 \& P60

Pretorius, Sarah - LBP11*

Prieto-Alhambra, Daniel - P1 \& P30

\section{$\mathbf{R}$}

Ranganath, Lakshminarayan - OC19 \& P2

Rankin, Kathryn - P53

Rashdan, Nabil - OC17 \& P23*

Redl, Heinz - P12

Redman, Amelia - LBP18*

Redmond, Niamh - OC4

Reid, Ian - LB2*

Riemen, Anna - LBP5

Roberts, Fiona - OC17*

Roberts, Scott - LBP11

Rochford, Justin - LBP14 \& LBP15

Rodriguez, Alexander - P27*

Rogers, Keith - P49

Rukuni, Ruramayi - P45

S

Samelson, Elizabeth J - LPB12

Sanchez-Santos, Maria T - P58

Sangar Mouse Pipelines - P13, P21 \& P22

Sano, Hiroshige - P35*

Saunders, Fiona - OC7, LBP4 \& LBP5

Schneider, Karl - P12 
Schneider, Philipp - OC14, P28 \& P52

SCOOP Study Team - OC4 \& OC16

Scott, David - P27

Segeletz, Sandra - LBP14

Shaw, Nick - P1

Shefelbine, Sandra J - P10

Shepstone, Lee - OC4 \& OC16

Shimakura, Taketoshi - P35

Sim, Alisia - P23

Singh Nijjar, Jagtar $-\mathrm{P}^{*}$

Skingle, Linda - P35 \& P42*

Snelling, Sarah - P8

Snowden, John - OC3 \& P17

Soper, Robin - P51

Sowman, Aneka - P58*

Sparkes, Penny - OC2, P13, P19, P21 \& P22

Spector, Tim D - P58

Spencer, Daniel - LBP3

Srikanth, Velandai - P27

Staines, Katherine - OC17, P24 \& P38

Steenhuis, Pieter - P39

Stephenson, Sonya - P31*

Stevens, Craig - P38

Stewart, Angela - LB2

Stocks, Oliver - P53

Swiatkowska, Agnes - OC2

Syddall, Holly - OC8

\section{$\mathbf{T}$}

Takahashi, Hideaki - P35

Tan, Juan - P7*

Tanaka, Sakae - P16 \& P6

Tang, Jonathan - OC15

Tare, Rahul S - LBP1

Tattersall, Luke - P62*

Tazzyman, Simon - OC3 \& P17

Tegenfeldt, Jonas - LBP3

Theobald, Peter - LBP6

Thomas, Neil Peter - OC19*

Thomson, Seamus - P56 \& P57

Tobias, Jon $\mathrm{H}-\mathrm{OC} 15$ \& P9

Torgerson, D - OC4

Townsend, Paul - P59

Tran, Anh - LBP14 \& LBP15

\section{U}

Ubago-Guisado, Esther - OC6

Udalova, Irina - P32

\section{V}

Van't Hof, Rob - OC18

Vanrenterghem, Gloria G - P40

Vaughan-Lane, Timothy - P42

Vlachopoulos, Dimitris - OC6*

\section{W}

Walker-Bone, Karen - P6

Ward, Kate - OC8, LB1, P4, P5 \& P60

Wells, Julia - P15

Westbury, Leo - OC8, P4* \& P5

Wheeler-Jones, Caroline - P44

White, Kate - OC12

Williams, Craig - OC6

Williams, Graham R - OC2, OC10, P13, P18, P21 \& P22

Williams, Katherine A - P52*

Wilson, Peter - P2

Wintsch, Emma - LBP7*

Woodfield, Tim - P48

Woods, Christopher - P53

Wright, Maria - P42

\section{$\mathbf{X}$}

Xavier, Miguel - LPB3*

Xu, Cecilia - P27

\section{$\mathbf{Y}$}

Yamada, Hiroshi - P6

Yamamoto, Noriaki - P35

Yang, Shoufeng - P14 \& P48

Yoshida, Munehito - P6

Yoshimura, Noriko - P16* \& P6

Yoshimura, Takashi - P18

Ytrebø, Lars Marius - P51

\section{Z}

Zakrzewski, Sonia - P53

Zioupos, Peter - P50 